Illinois State University

ISU ReD: Research and eData

Theses and Dissertations

3-14-2018

\title{
A Combinatorial Premotor Neural Code: Transformation Of Sensory Information Into Meaningful Rhythmic Motor Output By A Network Of Heterogeneous Modulatory Neurons
}

Christopher John Goldsmith

Illinois State University, christopher.goldsmith@gmail.com

Follow this and additional works at: https://ir.library.illinoisstate.edu/etd

Part of the Biology Commons, Neuroscience and Neurobiology Commons, and the Physiology Commons

\section{Recommended Citation}

Goldsmith, Christopher John, "A Combinatorial Premotor Neural Code: Transformation Of Sensory Information Into Meaningful Rhythmic Motor Output By A Network Of Heterogeneous Modulatory Neurons" (2018). Theses and Dissertations. 826.

https://ir.library.illinoisstate.edu/etd/826

This Dissertation is brought to you for free and open access by ISU ReD: Research and eData. It has been accepted for inclusion in Theses and Dissertations by an authorized administrator of ISU ReD: Research and eData. For more information, please contact ISUReD@ilstu.edu. 


\section{A COMBINATORIAL PREMOTOR NEURAL CODE: TRANSFORMATION OF SENSORY INFORMATION INTO MEANINGFUL RHYTHMIC MOTOR \\ OUTPUT BY A NETWORK OF HETEROGENEOUS \\ MODULATORY NEURONS}

\section{CHRISTOPHER JOHN GOLDSMITH}

\section{Pages}

The goal of the following research was to investigate the contributions of neural networks in selecting distinct variants of rhythmic motor activity. We used the premotor commissural ganglion ( $\mathrm{CoG}$ ) in the stomatogastric nervous system of the Jonah crab to understand how this network effectively controls the rhythms produced in downstream motor circuits. Prior research determined that individual CoG neurons are necessary to mediate sensory-induced variation in the effected motor patterns. However, single premotor neuron inputs to the STG are not sufficient to recreate the patterns induced by the selective activation of sensory pathways. Thus, it was hypothesized that the CoGmediated effects on these sensorimotor transformations may be explained at the level of CoG population activity.

We embraced the exploratory nature of this study by approaching it in three phases. First, we established voltage-sensitive dye imaging in the stomatogastric nervous system, as a technique that reports the simultaneous activity of many neurons with single-neuron resolution. In short, this form of imaging was effective at reporting both slow and fast changes in membrane potential, and provided an effective means of 
studying neuronal population activities. In addition, these dyes stain fine neural structures through nerve sheaths, connective tissue that must often be surgically removed to access the neurons contained within the sheaths. Then, we characterized the distribution of somata in the $\mathrm{CoG}$, and found that soma location was not fixed in its location from animal to animal, but that clustering of CoG somata did occur near their different nerve pathway origins. Finally, we used the voltage-sensitive dye-imaging technique to investigate the CoG population under different sensory conditions, and found that two different sensory modalities, one chemosensory and one mechanosensory pathway, differentially affected the balance of excited and inhibited (network activation) neurons found in the CoGs. Moreover, differences in CoG neuron participation between modalities was not extremely robust. However, the population activity differed enough so that both CoG participation and activation were drivers of the observed changes in the downstream pyloric motor network, providing support for a premotor combinatorial code for motor pattern selection.

KEYWORDS: stomatogastric; network; population activity; voltage-sensitive dye; fluorescence; electrophysiology; optical imaging; multimodal; premotor; central pattern generator; neuromodulation 


\section{A COMBINATORIAL PREMOTOR NEURAL CODE: TRANSFORMATIONS OF SENSORY INFORMATION INTO MEANINGFUL RHYTHMIC MOTOR OUTPUT BY A NETWORK OF HETEROGENEOUS MODULATORY NEURONS}

CHRISTOPHER JOHN GOLDSMITH

A Dissertation Submitted in Partial Fulfillment of the Requirements for the Degree of DOCTOR OF PHILOSOPHY

School of Biological Sciences ILLINOIS STATE UNIVERSITY 
(C) 2018 Christopher John Goldsmith 


\section{A COMBINATORIAL PREMOTOR NEURAL CODE: TRANSFORMATIONS OF SENSORY INFORMATION INTO MEANINGFUL RHYTHMIC MOTOR OUTPUT BY A NETWORK OF HETEROGENEOUS MODULATORY NEURONS}

CHRISTOPHER JOHN GOLDSMITH

COMMITTEE MEMBERS:

Wolfgang Stein, Chair

Joe Casto

Paul Garris

Craig Gatto

Andrés Vidal-Gadea 


\section{ACKNOWLEDGMENTS}

The readers of this section most likely understand that acquiring a PhD in biological sciences is no trivial task, and is most often a grueling process. For those who are less familiar with this, here are a few things. Attaining a graduate degree in any field is something in which to be proud. In general, it falls under a principle of which my dad often reminded me when dealing with hardship, "If it was easy, everyone would do it." Acquiring a higher-level degree in biology, and in particular as one esteemed ISU faculty member once said, "hardcore neuroscience", is filled mostly with strife with very brief glimpses of reward. I hear this phrase from non-academics that are familiar with academic research: "publish or perish." This is unfortunately and utterly true. No matter how much one publishes throughout the graduate career, we all gain a very special perspective. It is this peer-review process that makes one say, "good grief". It is also going through this process that allows one to grasp its importance, that is, having one's findings and theories solidified in the scientific literature must at least stand a test of legitimacy. It is unfortunate that I am writing this at a time where the credibility of information in general is in question. Thus, I think it is important for me to first acknowledge the altruistic scientist, one that rigorously tests specific predictions that will likely serve only as a small puzzle piece to more general scientific problems. The vast majority of scientists will not be world-renowned, but Jonas Salk and Albert Einstein would not be legendary without the vast majority of scientists that came before and after them. That is what we do and who we are, and for that reason, academic scientists have my eternal respect. 
Financial support was split between a grant (NSF IOS 1354932) acquired by my PI, Wolfgang Stein, and the School of Biological Sciences. These opportunities were integral to my completion of this dissertation research, but there are several other very important people whom I acknowledge in the paragraphs below.

For...Committees of faculty members...

I particularly would like to acknowledge those individuals comprising my PhD committee: Craig Gatto, Andrés Vidal-Gadea, Joe Casto, Paul Garris, and Wolfgang Stein. Craig has been the director of the school of biological sciences at ISU since before I started my graduate career. He has done and continues to do great things for our program. He stays busy, but if his door is open I have always been greeted in a hospitable manner. In a constant battle for funding, Craig has worked hard to not just keep our program afloat, but to improve it via the recruitment of impressive faculty and students. This is not a down-hill battle, and you have my support in years to come.

With our relatively small biological community here, every faculty member makes a difference. Andrés was definitely one of the impressive faculty recruits. To hear the term "magnetosensation", and that he was uncovering cellular mechanisms behind this drew excitement from me and I think from most in the department. It was in fact before he even arrived here that I asked him to be on my committee. Andrés also has allowed me to use his confocal microscope to address some reviewer concerns on one of my manuscripts, which was a huge help as this type of imaging is no commodity, and it was necessary for me to proceed with publication. 
For a period starting in my $4^{\text {th }}$ semester here, Joe and Paul acted as gracious hosts while I worked on a stint of dopamine experimentation. Under the guidance of Amanda Smith (former PhD student in Joe's lab), I picked up the technique of immunocytochemistry. With the help of Doug Schuweiler (PhD student in Paul's lab), I gained experience in fast-scan cyclic voltammetry. I would not call myself an expert neurochemist, but these experiences provided me with insight into how other labs function and scientific perspectives that will last a lifetime.

In the 5-plus years that I have been at ISU, I do not think I have seen anyone put in as much work as my PI and committee chair, Wolfgang Stein. Working on-site from 9to-9 is normal for him, and this does not include the near $24 / 7$ email availability. In short, getting ahold of him was never a problem. I do not think there was a time that I asked for help on something and he was not available within a day. Usually the response was instantaneous, unless he had prior obligations. This is a big deal. Wolfgang is always looking out for his students, via financial support, job prospects, or just insight on life. The first half of my time here, we had lunch almost every day. Sometimes we talked science, but this was a time we mostly discussed history and worldly issues. These conversations have been invaluable in shaping my outlook on the world.

It is also imperative that I recognize the support that I have received from Rosangela Follmann (and Epa Rosa!). The analytical aspects of this research would surely not exist without the work Rosangela has put forth. She also joined the lab at a time where I really needed a teammate and friend. Thank you for being there and helping me see past the issues that were right in front of my face! 
I have been somewhat of a hermit while in graduate school, but I still would like to acknowledge some of the friends that I have made here: Doug (wiser than I), Marissa (ratchet \#swerve), Ben (you mad bro?), Cody (N64 nutella), Sheed (you already know what it is), Josh (Mr. Neighbor Hero), AJ (makes a room smile), Kibbs (empathy strong with this one), Chad (citizen first), Jonny (hub neuron), Jack (come back after Canada), Nate (great approach), Maurice (I owe you 50 dollars), Marty (don't lie, I know your family owns Bobak's), Kevin (milk-chugging extraordinaire), and Justin J (better late than never). I will not forget any of these people. FFL.

For...Flightless family and friends...

I have spent the past 10 years floating around the Midwest, with a deep desire to take my life somewhere different. To be honest, it's the weather. As I write this in early Spring, we just got half a foot of snow, and the sky is chronically gray. It could be way worse though...I could be stuck in the Midwest without family and friends. Actually, I have a big family, and many friends, and I would vouch that they are the best family and friends on this planet (maybe I am bias). Most of them remain in the drab Midwest. It is calming to know that after all this time, they are still there supporting me as I scrape by. I look forward to spending the rest of my life with all of you, and am excited to commence the greatest journey on which I have ever embarked. In short, I am flat out lucky:

Justin...Don't quit snackin'...keep snackin'.

Nick...I think things are finally paying off, just keep the fires maintained.

Charlie...Never stop telling me how it is, sometimes I forget. Kyle...Come back from Oregon. 
Dom... Your collage of perspectives is priceless.

Michal...Always there to help.

John... Hero Clicks will always be cool. Cooler than protein biochemistry at least.

Tommaso...Bees make honey, but they also retain ecosystem functionality.

Mitch...Bear Grillz is pretty awesome.

Faulks...Well, okay then.

Weiner...Slick Rick!

Woods...But that's my dust.

Tyler...Actuarial science is actually pseudoscience.

Aunts, uncles, cousins...to me, you are more like mothers, fathers, and siblings.

Alex...Never stop writing.

Greg...Always have and always will look up to you.

Becky...Let's make that app.

Peter...I hope you can always look up to me.

Eric... Hot water burn baby.

Kristina...It's not my fault my facial hair grows the way it does.

Matt B...Everything irie.

Nicole...Composting is great, and chem-trails I hate.

Sandy...You have unleashed the vape monster.

Olivia...I can't tell if you are being sarcastic, sometimes.

Natalie...I want to meet your Hollywood friends.

Jim...Well excuse me for telling you exactly what you did.

Matt W...We used C++ because it's faster, not better. 
Renee...You can feed Izzy as long as Irie does not see it.

Matt K...Come hang out more often.

Michelle...Come hang out more often!

Jon...I used to be better at video games, I swear.

Jillian...Be smart, stay out of trouble.

Mom, Dad, Alisse...Since the beginning, until the end, best family ever. It is a huge deal to know that even as I approach 30 , that I can always turn to you all for help. Most people do not have that type of support. You should know that it is reciprocal, though. Anytime.

Sam...Thank you for propping me up over the last few years. I have been somewhat of a Weeble Wobbling. It is awesome how overall we can remain so positive when faced with adversity. Later in life when we are complaining about something, we should look back to now, and remember that when things are hard, laughter helps. Dogs help as well! Gets better from here!

To bring this full-circle...I really have nothing to complain about, and have the utmost gratitude for the people mentioned above. The following stanzas represent some concepts that I have critically assessed over my duration in graduate school, and in short, are worthy of acknowledgement despite their intangibility and non-human content. Though, they are important to humans in general: 
For...

Academia... an emergent property of society, and societal values in education. Presumably the traces of bureaucracy are woven so sparsely into academia that it generally maintains an active and modest, yet harmonious exchange of information. A privilege for any society... a necessity for the canonical society.

For...

Freedom of information... specifically, freedom for one to exchange information openly and choose the information with which one is exposed. These principles are exclusionary only in-part. But, instances involving an overly problematic manifestation of their paradoxy should be addressed on a case-by-case basis. Perhaps these things are not self-evident.

For...

The academic scientist... whose [more or less] altruistic approach to discovery rigorously and truthfully investigates the most fascinating questions of the universe. Slowly, gathering flakes of empirical gold. The vast majority of scientists are not worldrenowned, and very few "strike gold". But excellent goldsmiths like Jonas Salk and Albert Einstein would not be the renowned individuals they turned out to be without the vast majority of scientists who came before and after them. 
For...

Innate human curiosity...its ontogeny irrevocably confounded without untangling its phylogeny....in itself a spacetime-bound trajectory from which WE emerged...destiny. Whatever it all means, it can only happen one way, it will only happen one way, we will never know how it will happen until after it happens, and there is absolutely nothing we can do about it, except ignore it...

And be curious.

For...

The cortex...because it turns out that selection for more brain cells was the evolutionary driving force that ultimately allowed us to image things with confocal microscopes, rather than be imaged with a confocal microscope.

But of course, for...

The neuron...for what better way is there to understand something than to take it apart...Breaking something into roughly a trillion components, however, surely puts the prior saying to a test-of-scale. If anything then, it must be for...job security.

... and the ability to acknowledge one's own privilege in being able to investigate critical scientific concepts...

C.J.G. 


\section{CONTENTS}

Page

ACKNOWLEDGMENTS i

$\begin{array}{ll}\text { CONTENTS } & \text { ix }\end{array}$

CHAPTER I FIGURES Xiii

CHAPTER II FIGURES Xiv

CHAPTER III FIGURES X XV

CHAPTER I: THE UTILIZATION OF VOLTAGE SENSITIVE DYES IN

ILLUMINATING FINE STRUCTURES AND REPORTING NEURONAL

POPULATION ACTIVITY: A CASE STUDY ON NERVOUS SYSTEM SHEATHS 1

$\begin{array}{ll}\text { Abstract } & 1\end{array}$

$\begin{array}{lr}\text { Introduction } & 2\end{array}$

$\begin{array}{ll}\text { Methods } & 5\end{array}$

$\begin{array}{ll}\text { Dissection } & 5\end{array}$

$\begin{array}{ll}\text { Extracellular recording } & 6\end{array}$

$\begin{array}{ll}\text { Stimulation parameters } & 7\end{array}$

$\begin{array}{ll}\text { Preparation and application of the dyes } & 7\end{array}$

$\begin{array}{ll}\text { Optical imaging and picture processing } & 8\end{array}$

$\begin{array}{ll}\text { Data analysis } & 9\end{array}$

$\begin{array}{ll}\text { Statistics and figure making } & 10\end{array}$

$\begin{array}{ll}\text { Results } & 11\end{array}$

RH795 is a good anatomical marker for cell body location $\quad 11$

RH795 reveals fine neuronal structures like neurites, axons, and varicosities 
Desheathing the STG causes subtle changes in neuronal activity

$\mathrm{RH} 795$ does not alter neuronal activity in the STG

Neuronal activity can be imaged through the ganglion sheath

Simultaneous recordings of multiple gastric mill motor neurons through the ganglion sheath

Discussion

$\mathrm{RH} 795$ as an anatomical marker for living neural tissue

$\mathrm{RH} 795$ can be used to monitor neuronal activity through nerve sheaths

Functional implications

REFERENCES

FIGURES

CHAPTER II: SPATIAL DISTRIBUTION OF INTERMINGLING POOLS OF PROJECTION NEURONS WITH DISTINCT TARGETS: A 3-DIMENSIONAL ANALYSIS OF THE COMMISSURAL GANGLIA IN CANCER BOREALIS

Abstract

Introduction

Methods

Dissection

Staining of neuronal membranes

Retrograde labeling

Optical imaging and picture processing

Figures and data analysis 
General structure of the commissural ganglion

Descending projection neuron distribution in the commissural ganglion 64

$\begin{array}{ll}\text { Organization of projection neuron clusters } & 66\end{array}$

$\begin{array}{ll}\text { Spatial preferences of dPNs } & 69\end{array}$

$\begin{array}{ll}\text { Discussion } & 72\end{array}$

The commissural ganglia: Centers for controlling divergent neuronal outputs 72

Clustering and variability of projection neuron location 75

$\begin{array}{ll}\text { Preferential locations of projection neurons } & 78\end{array}$

$\begin{array}{ll}\text { REFERENCES } & 82\end{array}$

$\begin{array}{lr}\text { FIGURES } & 93\end{array}$

CHAPTER III: A PREMOTOR NETWORK ENCODES MULTIMODAL

INFORMATION AND CONTROLS MOTOR OUTPUT VIA A COMBINATORIAL

$\begin{array}{lr}\text { CODE } & 102\end{array}$

$\begin{array}{ll}\text { Abstract } & 102\end{array}$

$\begin{array}{ll}\text { Introduction } & 102\end{array}$

$\begin{array}{ll}\text { Methods } & 106\end{array}$

$\begin{array}{ll}\text { Animals } & 106\end{array}$

$\begin{array}{ll}\text { Solutions and reagents } & 107\end{array}$

$\begin{array}{ll}\text { Anterograde tracing of sensory pathways } & 107\end{array}$

$\begin{array}{ll}\text { Application of a voltage-sensitive dye } & 107\end{array}$

$\begin{array}{ll}\text { Stimulation of sensory nerve pathways } & 108\end{array}$

$\begin{array}{ll}\text { Optical imaging } & 108\end{array}$

$\begin{array}{ll}\text { Extracellular nerve recordings } & 109\end{array}$ 
Data analysis and figure construction

Imaging analysis

Motor pattern analysis

Results

The CoG premotor region processes information from multiple sensory modalities

Similarities in the premotor spike frequency distributions between modalities contradict a network rate code

Modality-specific neurons and distinct premotor network activation support a combinatorial code for multimodal representation

The bimodal sensory condition elicits mechanosensory-like premotor network activation and participation

CoG premotor network activation and participation contribute to downstream changes in pyloric motor output

Discussion

Potential mechanisms for the encoding of multimodal sensory information

A combinatorial code of premotor network activation and participation

determines motor output

The bimodal condition is encoded in a new combination of premotor activities 131 Implications for multimodal encoding and motor pattern selection in higher order brain areas

REFERENCES

FIGURES 


\section{CHAPTER I FIGURES}

Figure

Page

1. Schematic overview of the stomatogastric nervous system

2. RH795 stains through the ganglion sheath and allows visualization of soma location

3. RH795 staining in the stomatogastric nervous system of the crab 46

4. Desheathing causes subtle changes in neuronal activity 48

5. Optical imaging through the ganglion sheath 50

6. Repetitive staining permits long-term experiments 51

7. RH795 allows the simultaneous recording of gastric mill neurons through the ganglion sheath 


\section{CHAPTER II FIGURES}

Figure

Page

1. Schematic representation of the C. borealis STNS 93

2. Description of CoG neuron location in a single preparation 94

3. General description of CoG somata 95

4. CoG neurons projecting to the STG are mostly posteriorly and medially located

5. Neurons with distinct functions and targets overlap in soma localization 97

6. Soma distribution of CoG neurons projecting to the TG and brain 98

7. CoG neurons projecting via different pathways overlap in their locations 99

8. Soma cluster centroids are spatially weighted towards their respective pathway origins

9. Variability of dPNs location within the CoG 


\section{CHAPTER III FIGURES}

Figure

Page

1. Voltage-sensitive dye imaging in a multifunctional motor system 147

2. Multiple sensory modalities converge onto the commissural ganglion 149

3. Comparison of optical and intracellular recordings 150

4. Neuronal response latencies and spike frequencies are similar between sensory modalities

5. Network excitation and inhibition is modality-specific

6. No differences in the spatial distributions of premotor CoG neurons were found between different sensory conditions

7. Bimodal and mechanosensory stimuli yield similar premotor network activation and participation

8. Unimodal chemosensory and mechanosensory inputs yield functionally different pyloric motor patterns

9. Premotor network participation is related to the differences in pyloric motor output across sensory conditions

10. CoG premotor network activation is related to the change in downstream pyloric activity

11. CoG network response to bimodal sensory stimulation differs from expected outcomes 
CHAPTER I: THE UTILIZATION OF VOLTAGE SENSITIVE DYES IN ILLUMINATING FINE STRUCTURES AND REPORTING NEURONAL POPULATION ACTIVITY: A CASE STUDY ON NERVOUS SYSTEM SHEATHS

\begin{abstract}
Locating circuit neurons and recording from them with single-cell resolution is a prerequisite for studying neural circuits. Determining neuron location can be challenging even in small nervous systems because neurons are densely packed, found in different layers, and are often covered by ganglion and nerve sheaths that impede access for recording electrodes and neuronal markers. We revisited the voltage-sensitive dye $\mathrm{RH} 795$ for its ability to stain and record neurons through the ganglion sheath. Bathapplication of RH795 stained neuronal membranes in cricket, earthworm and crab ganglia without removing the ganglion sheath, revealing neuron cell body locations in different ganglion layers. Using the pyloric and gastric mill central pattern generating neurons in the stomatogastric ganglion (STG) of the crab, Cancer borealis, we found that $\mathrm{RH} 795$ permeated the ganglion without major residue in the sheath and brightly stained somatic, axonal and dendritic membranes. Visibility improved significantly in comparison to unstained ganglia, allowing the identification of somata location and number of most STG neurons. RH795 also stained axons and varicosities in nondesheathed nerves, and it revealed the location of sensory cell bodies in peripheral nerves. Importantly, the spike activity of the sensory neuron AGR, which influences the STG motor patterns, remained unaffected by $\mathrm{RH} 795$, while desheathing caused significant changes in AGR activity.
\end{abstract}


With respect to recording neural activity, $\mathrm{RH} 795$ allowed us to optically record membrane potential changes of sub-sheath neuronal membranes without impairing sensory activity. The signal-to-noise ratio was comparable with that previously observed in desheathed preparations and sufficiently high to identify neurons in single-sweep recordings and synaptic events after spike-triggered averaging.

In conclusion, $\mathrm{RH} 795$ enabled staining and optical recording of neurons through the ganglion sheath and is therefore both a good anatomical marker for living neural tissue and a promising tool for studying neural activity of an entire network with singlecell resolution.

\section{Introduction}

An important prerequisite for studying the properties and connectivity of neural circuits is to locate the same neuron or classes of neurons in each preparation. However, even in nervous systems with a small number of neurons, determining neuron location can be challenging because neurons are densely packed and found in different layers, and the nervous system is often covered by a protective sheath. Another challenge in identifying circuits is to simultaneously record the activities of the circuit neurons. Besides field potential recordings and the use of multi-electrode arrays, optical imaging with either expressed or bath-applied calcium- or voltage-indicators is often the preferred method (for reviews see (Cohen and Salzberg, 1978; Chemla and Chavane, 2010; Kim and Jun, 2013)). Yet, in systems where expression is not possible the success of the dye application is impeded by the presence of a ganglion sheath or connective tissue that prevents dye permeability (Baker et al., 2005). Consequently, tissue slices are taken, or the ganglion sheath and connective tissue are removed by microsurgery or enzymatic 
treatment before dye application, procedures both difficult and potentially harmful to the neurons (Briggman et al., 2005, 2006; Briggman and Kristan Jr., 2006). In the thoracic ganglia of the phasmid Extatosoma tiaratum, for example, surgically removing the sheath results in a loss of hydrostatic pressure inside the ganglion and a bulging out of the neurons (Debrodt and Bässler, 1989). Even when desheathing is possible, it is timeintensive, requires specific protocols (enzymatic approach) and/or many months of training in microsurgery (Galizia et al., 1997; Briggman and Kristan Jr., 2006; Gutierrez and Grashow, 2009; Hill et al., 2012). There is always the potential of damaging neurons, leading to a rather significant failure rate of the desheathing process in some systems. Even when the procedure is successful, the influence of removing the sheath on the neural activity is often unclear because a direct comparison of neural activity before and after desheathing is missing. This is despite the fact that there is evidence that the ganglion sheath can affect interstitial voltage and ion concentrations (Dörr et al., 1996).

Here, we are revisiting the voltage-sensitive dye (VSD) $\mathrm{RH} 795$ for its ability to stain neurons through the ganglion sheath. We found that bath-application of $\mathrm{RH} 795$ specifically stains membranes of neurons and axons without removing the ganglion sheath. We tested various nervous systems (cricket, earthworm, and crab), and in all cases we observed that $\mathrm{RH} 795$ had a stronger affinity for neural membranes than to the ganglion sheath, such that the sheath itself was not strongly stained. Besides being an extraordinary anatomical marker for cell membranes, $\mathrm{RH} 795$ allowed us to record neuronal activity through the sheath. For this, we used the stomatogastric ganglion (STG) of the crab Cancer borealis, a classic model for studying neural circuit 
connectivity and neuromodulation on the single cell level. In the STG, the ganglion sheath is typically removed via microsurgery to enhance visibility in the ganglion and facilitating intracellular recordings. Desheathing is also necessary to allow some of the most commonly used voltage-sensitive dyes (for example ANEP dyes) to label neural membranes in the STG (Städele et al., 2012).

The STG contains the pyloric and gastric mill central pattern generators (CPGs, which control the filtering and chewing of food, respectively), and like other CPGs (Brown, 1911; Grillner et al., 1998; Rossignol et al., 2006; Marder and Bucher, 2007; Dubuc et al., 2008; El Manira and Shenoy, 2009; Harris-Warrick, 2011), they produce regular and predictable oscillatory activities even in isolated preparations. Both CPG circuits in the STG have been characterized in great detail (Stein, 2009; Blitz and Nusbaum, 2011; Harris-Warrick, 2011) using mostly traditional extra- and intracellular electrophysiology after desheathing. The gastric mill CPG is influenced by sensory pathways, such as the proprioceptive anterior gastric receptor (AGR: Daur et al., 2009). Even small changes in spontaneous AGR activity cause significant changes in the activity of the gastric mill motor neurons (Daur et al., 2009). We found that desheathing the STG caused subtle changes in neural activity, namely that rhythmic oscillations in the firing frequency of AGR can no longer be observed after the ganglion is desheathed. $\mathrm{RH} 795$, however, specifically stained the membranes of the pattern generating neurons in the STG without removing the ganglion sheath, allowing not only the visual identification of all neuronal somata in the STG, plus several axons, but also optical recording from individual pattern generating neurons whose activities are influenced by AGR. Since activities were recorded through the sheath, no mechanical stress had 
been imposed during desheathing and AGR's rhythmic activity persisted. Thus, bathapplication of $\mathrm{RH} 795$ is a noteworthy tool for locating and recording many neurons simultaneously in living tissues, without the need for removing neural sheaths.

\section{Methods}

Dissection

Adult crabs (Cancer borealis) were delivered from The Fresh Lobster Company (Gloucester, MA, USA) or Ocean Resources Inc. (Sedgwick, ME, USA). Invertebrate animals used in research are not subject to ethics approval at Illinois State University, and Cancer borealis is not a protected species. We adhered to general animal welfare considerations regarding humane care and use of animals while conducting our research. Crabs were kept in tanks with artificial sea water (salt content $\sim 1.025 \mathrm{~g} / \mathrm{cm}^{3}$ ) made from artificial sea salt (Instant Ocean Sea Salt Mix, Blacksburg, VA, USA) for a maximum of 16 days. Tanks were kept at a temperature of $10-12{ }^{\circ} \mathrm{C}$ and a 12 -hour light-dark cycle. Before dissection, animals were anesthetized on ice for 20 minutes (Gutierrez and Grashow, 2009). Adult crickets (Gryllodes sigillatus) were a gift from Scott Sakaluk (Illinois State University, Normal, IL) and earthworms (Eisenia hortensis) were bought at a local bait shop. We used isolated nervous systems to perform all of our experiments. In short, the nervous system was pinned down in a silicone elastomerlined (ELASTOSIL RT-601, Wacker, Munich, Germany) Petri dish and continuously superfused $(7-12 \mathrm{ml} / \mathrm{min})$ with saline $\left(10-13^{\circ} \mathrm{C}\right.$ for $\mathrm{C}$. borealis, room temperature for G. sigillatus and E. hortensis). Physiological crab saline consisted of: $\mathrm{NaCl}, 440 \mathrm{mM}$; $\mathrm{KCl}, 11 \mathrm{mM} ; \mathrm{MgCl}_{2} \cdot 6 \mathrm{H}_{2} 0,26 \mathrm{mM} ; \mathrm{CaCl}_{2}, 13 \mathrm{mM}$; trisma base, $10 \mathrm{mM}$; maleic acid, 5 $\mathrm{mM}$ (pH 7.4 - 7.6). Insect saline consisted of: $\mathrm{NaCl}, 187 \mathrm{mM} ; \mathrm{KCl}, 21 \mathrm{mM} ; \mathrm{CaCl}_{2}, 5.6$ 
$\mathrm{mM} ; \mathrm{MgCl}_{2} \cdot 6 \mathrm{H}_{2} 0,4.1 \mathrm{mM}$ and earthworm saline consisted of: $\mathrm{NaCl}, 103 \mathrm{mM} ; \mathrm{KCl}, 1.6$ $\mathrm{mM} ; \mathrm{CaCl}_{2}, 1.4 \mathrm{mM} ; \mathrm{NaHCO}_{3}, 1.2 \mathrm{mM}$. Crabs were sacrificed on ice, and crickets and earthworms using $100 \%$ Ethanol. Both methods are recognized as acceptable under the AVMA guidelines for euthanasia of invertebrates.

\section{Extracellular recording}

We used the stomatogastric nervous system (STNS) of $C$. borealis to perform activity measurements. Neuronal activity was recorded extracellularly on one of the main motor nerves. The generalized recording setup is shown in fig. 1. For monitoring the pyloric rhythm we recorded either the lateral ventricular nerve (Ivn) or the dorsal ventricular nerve (dvn); for the gastric mill rhythm we recorded DG and the GMs extracellulary on the dorsal gastric nerve (dgn) and LG on the lateral gastric nerve (Ign) (fig. 1). The gastric mill cycle period was defined as the duration between the onset of an impulse burst in LG and the onset of the subsequent LG burst. AGR activity was assessed with extracellular recordings of the dgn, stn (stomatogastric nerve) and/or son (superior oesophageal nerve). AGR activity was measured as instantaneous firing frequency (inst. ff.) as determined by reciprocal of the interspike interval. Mean values for all gastric mill-related parameters were determined from measurements of 20 consecutive cycles of gastric mill activity. For phase analysis, AGR inst. ff. was normalized to the minimum and maximum frequencies measured in each cycle. In some experiments the pyloric dilator nerve $(p d n)$, which contains the axons of the two pyloric dilator (PD) neurons, was recorded in addition. The PD neurons are part of the pacemaker ensemble of the pyloric circuit (Stein, 2009). We used petroleum jelly wells and subsequent measurements of field potential changes between two stainless steel wires 
(one inside and one outside of each well) to extracellularly record action potentials. The differential signal was recorded, filtered and amplified with an AC differential amplifier (A-M Systems Modell 1700, Carlsborg, WA, USA). Files were recorded, saved and analyzed using Spike 2 Software (version 7.11; CED, Cambridge, UK).

\section{Stimulation parameters}

To show the rhythmic modulation of the AGR inst. ff. we elicited a gastric mill rhythm. For this, the ventral cardiac neurons (VCNs) were activated by stimulating the dorsal posterior oesophageal nerve (dpon) extracellularly with 10 consecutive stimulus trains of $15 \mathrm{~Hz}$ stimulation frequency, $6 \mathrm{~s}$ train durations and $4 \mathrm{~s}$ intertrain intervals (Beenhakker et al., 2004).

\section{Preparation and application of the dyes}

In all experiments we used the styryl dye RH795 (Pyridinium, 4-[4-[4(diethylamino)phenyl]-1,3-butadienyl]-1-[2-hydroxy-3-[(2hydroxyethyl)dimethylammonio]propyl]-,dibromide/172807-13-5; Biotium, Hayward, CA) which was first synthesized by Rina Hildesheim and Amiram Grinvald (Grinvald et al., 1994). A $10 \mathrm{mM}$ stock solution was prepared by diluting $5 \mathrm{mg}$ dye in $854 \mu \mathrm{l}$ of ultrapure water and kept in darkness at $4{ }^{\circ} \mathrm{C}$. Immediately before bath-application, the stock solution was diluted in saline to the final concentrations of $0.3 \mathrm{mM}$. Fig. 1 shows the application setup for the STG. Application procedures were similar for cricket and earthworm ganglia. In contrast to most other studies, we did not desheath the STG for performing the optical recordings. Rather, a petroleum jelly well that isolated the STG from other parts of the STNS was built and approximately $50 \mu \mathrm{l}$ of $\mathrm{RH} 795$ were bathapplied to the well (see Preuss and Stein, 2013). The part of the STNS that was located 
on the outside of the well was constantly superfused with chilled saline $\left(10-12{ }^{\circ} \mathrm{C}\right)$ during dye application. The dye was applied for $30-60$ minutes, after which the petroleum jelly well was removed and the whole preparation was superfused with chilled saline for the remainder of the experiment. For comparing the visibility between stained/non-desheathed and stained/desheathed ganglia, we desheathed the STG and took a count of the visible cells in some experiments.

Optical imaging and picture processing

For comparing the fluorescence before and after desheathing, we used a 5 mega pixel color CMOS camera (TCA-5.0C, Ample Scientific LLC, Norcross, GA, USA) and TSView software (Version 7.3.1.7, Tucsen Imaging Technology Co., Fujian, China). Fluorescent excitation light was provided by a CoolLED system (narrowband LED with $535 \mathrm{~nm}$; Yorktown Heights, NY) and fluorescence emission was detected using a 560 nm beam splitter and a 570 - 640 nm emission filter (Olympus, Center Valley, PA). Excitation light intensities and imaging exposure time varied and were adjusted to the individual preparation. We either used a 20 x objective (XLUMPlanFL N, NA 1.0, WD $2.0 \mathrm{~mm}, \mathrm{cc}=$ water; Olympus Corporation, Tokyo, Japan) or a $10 \mathrm{x}$ objective (UMPlanFL N, NA 0.30, WD 3.3 mm, cc=water; Olympus Corporation, Tokyo, Japan) mounted on an upright epifluorescence microscope (modified BX51, Scientifica, East Sussex, UK). For recording fluorescence changes ('optical imaging') the MiCam02 imaging system and software (Brain-Vision Analyzer, BV-ANA, Version 11.08.20; SciMedia Ltd, Tokyo, Japan) were used with the HR (High Resolution) camera $(6.4 \times 4.8 \mathrm{~mm}$ actual sensor size) set at either $384 \times 256$ pixel spatial resolution for high resolution photos or at $192 \mathrm{x}$ $128,96 \times 64$, or $48 \times 32$ pixel spatial resolution for optical imaging. A temporal 
resolution of 2-20 ms was chosen. Typical recordings lasted between 16 and 32 seconds, and were repeated many times in a given experiment.

Data analysis

Averaging of signals was performed using associated scripts for Spike2 (www.neurobiologie.de/spike2). The cycle period of the pyloric rhythm was defined as the duration between the onset of a PD neuron burst and the onset of the subsequent PD burst. In some experiments, cycle-based and spike-triggered averaging were used to improve signal quality, according to the protocols given in (Städele et al., 2012) and analyzed in Spike2. Cell counts were performed in dark-field illumination using a Wild M8 stereomicroscope (Heerbrugg, Switzerland) or an upright epifluorescence microscope (modified BX51, Scientifica, East Sussex, UK), before and after desheathing and before and after staining with $\mathrm{RH} 795$.

In selected experiments, the frequency components of optical and extracellular signals were plotted as a spectrogram. In these cases, frequency analysis was restricted to a frequency band of $0-10 \mathrm{~Hz}$, which contains the main frequencies present during the pyloric and gastric mill rhythms. Additionally, the correlation of the frequency distribution was calculated for each point in time during the recordings, allowing the direct comparison of the frequency components of different neurons. This allows for a quantification of the correlative strength of activity from neuron-to-neuron, along with showing if a given neuron participates in a particular rhythm at all times. Time resolution was 0.1 seconds and frequency resolution was $0.1 \mathrm{~Hz}$ (resulting in 100 frequency steps from $0-10 \mathrm{~Hz}$ that were correlated at each time point). 
Waveform correlations were calculated by multiplying two waveforms together, point by point, and summing the products. The sum was normalized to allow for waveform amplitudes and the number of points. The reference waveform was then repeated for all time bins. Results range between +1.0 , meaning the waves are identical except for amplitude through 0 (uncorrelated) to -1.0 , meaning identical but inverted. The bin width corresponded to the sampling bin width.

Optically recorded neurons were identified after cycle-based averaging by comparing waveform shape, phasing, and timing of action potential occurrence relative to extracellular recordings (similar to the identification of intracellularly recorded neurons).

Statistics and figure making

For spreadsheet analysis, Excel (version 2010 for Windows, Microsoft) or SigmaPlot (version 11 for Windows, Systat Software GmbH, Erkrath, Germany) were used. Normally distributed data are given as mean \pm SD. "N" denotes the number of animals, while " $n$ " is the number of trials. In all figures significance is indicated using * $(p<0.05)$, ${ }^{* *}(p<0.01),{ }^{* * *}(p<0.001)$. Statistical tests for data analysis were t-test, Pearson product-moment correlation coefficients or One Way ANOVA with Holm Sidak posthoc test. Final figures were prepared with CorelDraw (version X3 for Windows, Corel Corporation, Ottawa, ON, Canada). 


\section{Results}

One of the major advantages of small system approaches in neuroscience is the use of identified neurons or neuronal populations (Nusbaum and Beenhakker, 2002;

Williamson and Chrachri, 2004; Kristan Jr. et al., 2005). However, recordings are often hindered by (a) the difficulty to localize neuron somata and arborizations in living tissue and (b) having to penetrate neurons through non-neural tissue such as the ganglion sheath in most invertebrate preparations. Here, we revisit $\mathrm{RH} 795$, a voltage-sensitive dye used for optical imaging, and assesses its ability (a) to stain populations of neurons in living tissue and (b) to allow recording through the ganglion sheath. We used three different model systems with partly identified neural circuits to test RH795: the cricket metathoracic ganglion, the earthworm ventral ganglion chain, and the crustacean stomatogastric nervous system.

RH795 is a good anatomical marker for cell body location

After dissection, the isolated nervous system was placed into a Petri-dish containing physiological saline. For the control, the ganglion was photographed in white light before $\mathrm{RH} 795$ was bath-applied. $\mathrm{RH} 795$ was left on for one hour and subsequently washed out with saline. Fluorescence imaging began 15 minutes after the washout was started. We found similar results for all ganglia used: before dye application, very few, if any, neuronal somata or arborizations were visible. Fig. 2A shows a cricket metathoracic ganglion before and after dye application. As in most insect thoracic ganglia, the cricket ganglion has distinct areas for neuropil structures and cell bodies, respectively (Insausti et al., 2011). This broad distinction was visible before staining: lighter areas around the outline of the ganglion, where the cell bodies are located, and 
denser neuropil areas in the middle of the ganglion. However, no individual neural structures could be discerned. After dye application, the outlines of many neuronal somata were visible in the fluorescent light. Moving the focal plane of the microscope from dorsal to ventral revealed multiple layers of neurons that were all individually identifiable (arrows in figs. 2A(i)-(iii)).

We found similar results for the staining of the earthworm ventral ganglia (fig. 2B): before dye application, very few individual cell bodies were visible. After staining with $\mathrm{RH} 795$ the outlines of distinct neuronal somata were evident, and in particular at the lateral borders, where large neuronal somata are located (Telkes et al., 1996). As for the cricket ganglion, changing the focal plane revealed the location of different distinct neuronal layers.

In contrast to most other arthropod ganglia, the STG of the crab, Cancer borealis, contains only 26 neurons, all of which are individually identifiable by their firing and axonal projection pattern (Stein, 2009). Due to the extensive knowledge about the circuit connectivity and the neural activity, we used the STG to study RH795 in more detail. In none of the crustacean species studied are the location of STG neurons fixed, i.e. neuron location differs from animal to animal (Bucher et al., 2007). Hence, the desheathed ganglion is typically used to increase visibility in the ganglion and to facilitate access to the neurons. Indeed, desheathing improved visibility in our experiments as well (fig. $3 \mathrm{~A})$ : On average, the location of $10.3 \pm 2.9$ somata $(\mathrm{N}=15)$ could be determined before desheathing. After desheathing the number of unambiguously identifiable neurons increased significantly to $19.8 \pm 3.2(\mathrm{~N}=55, \mathrm{p}<0.001$; One Way ANOVA with Holm Sidak posthoc test). Voltage-sensitive dyes have been used to study 
small invertebrate nervous systems for some decades (primarily absorption VSDs (Salzberg et al., 1973, 1977)). We have previously shown that fluorescent VSDs, such as ANEP dyes (Obaid et al., 2004; Wang et al., 2009) and RH795 (Preuss and Stein, 2013), stain neural membranes in the STG and allow the visual recognition of most STG neurons (Städele et al., 2012; Preuss and Stein, 2013). However, VSDs are typically applied after removing the ganglion sheath (Galizia et al., 1997; Obaid et al., 2004; Baker et al., 2005; Briggman et al., 2005; Briggman and Kristan Jr., 2006; Chemla and Chavane, 2010; Stein and Andras, 2010; Stein et al., 2011; Hill et al., 2012). Here, as for the cricket and earthworm, we bath-applied $\mathrm{RH} 795$ to the non-desheathed ganglion to test its ability as a neural marker without removing the ganglion sheath. $\mathrm{RH} 795$ was bath-applied for 60 minutes, after which dye washout was started and another cell count was taken. At this point in time, the sheath still contained large amounts of dye, obscuring some of the fine details of the intra-ganglionic structures (fig. 3B, left). Nevertheless, most of the 26 STG cell bodies could be located $(21.2 \pm 3.6, N=21$, significantly different from before desheathing/not stained, $p<0.01$; One Way ANOVA with Holm Sidak posthoc test). Contrast and visibility improved within the next 20 minutes, after which a stable staining was observed for at least the following hour and up to 3 hours (fig. 3B). Apparently, the dye had stained through the ganglion sheath without excessive residual staining of the sheath. To confirm that indeed neuronal membranes had been stained, we desheathed the ganglion after the staining procedure and compared the visibility before and after desheathing (fig. 3B, right). There were no obvious differences in location, size and shape of the neurons, and the number of neurons that could be accounted for by visual inspection of the ganglion was not 
significantly different from the non-desheathed/stained ganglia $(19.5 \pm 4.8 ; N=6 ; p>0.2$; One Way ANOVA with Holm Sidak posthoc test).

RH795 reveals fine neuronal structures like neurites, axons, and varicosities RH795 also revealed individual axons in the STG (fig. 3C (i)), which typically cannot be seen without fluorescence staining even when the ganglion is desheathed (for comparison see fig. 3A (ii)). Fig. 3C (i) shows the anterior part of the STG, where the stn enters the STG. The stn connects the STG to three other ganglia as well as to the rest of the CNS and contains approximately 60 axons (Coleman et al., 1992). A striking feature is that axons that enter the STG tend to be larger in diameter and more spread out than they are in middle parts of the same nerve. For example, axons cover over a width of $\sim 200 \mu \mathrm{m}$ when they enter the STG, while they are bundled and stacked and take up less than $100 \mu \mathrm{m}$ in the middle of the stn. After staining with RH795 stn axons were clearly visible, both in the STG and in the stn. In some cases, we could even identify specific axons: fig. 3C(i) shows an axon entering the STG via the stn and leaving the STG (arrows 1 and 2) via one of the side nerves (aln). This is the axon of the CD1 neuron (cardiac sac dilator neuron 1), the only neuron that projects an axon via the stn to the aln (Vedel and Moulins, 1977; Dickinson and Marder, 1989). RH795 also revealed structures usually invisible even after desheathing, such as axons lying on top of neurons (arrow 3 ) and the shape of primary neurites (dotted square). We tested whether individual axons could also be traced in nerves with bundled axons by bathapplying $\mathrm{RH} 795$ to individual nerves. We compared nerves containing densely bundled axons (middle part of the stn) with motor nerves containing only a few axons ( $p d n)$ and sensory nerves (gpn: gastro pyloric nerve (Katz et al., 1989) and ivn: inferior ventricular 
nerve (Hedrich et al., 2009). We used the same procedure as for the ganglion staining. In general, we found that $\mathrm{RH} 795$ clearly stained axon bundles without strong residual staining of the nerve sheath. Staining the middle part of the stn revealed many individual axons and the staining was good enough to trace a particular axon over a large distance (fig. 3C(ii)). In addition, several varicosities could be seen in the vicinity of the stn axons (arrows in fig. 3C(ii)). Identifying individual axons was easier in nerves containing only a few axons, such as the ivn. The ivn contains only 8 axons (Hedrich et al., 2011), namely those of two identified projection neurons and 6 axons of so far undescribed neurons. After staining with $\mathrm{RH} 795$ we could clearly separate 6 of the 8 axons from each other (fig. 3C(iii)), even in a single optical focal plane. Stainings of the pdn (fig. 3C(iv)), through which the two PD neurons project to the pyloric muscles, revealed two large axons plus at least one small diameter axon (possibly sensory). In the case of the gpn (fig. $3 \mathrm{C}(\mathrm{v})$ ), the dye revealed not only individual axons, but also the location of the soma of the gastro-pyloric receptor neuron (Katz and Harris-Warrick, 1991), a muscle stretch receptor, which so far has only been located with retrograde backfill stainings in fixed tissues (Katz et al., 1989). Finally, RH795 allowed us to visualize the soma of the sensory neuron AGR (Smarandache and Stein, 2007) with relative ease (fig. 3D). The AGR soma is located in the posterior part of the STG, separated from the motor neurons. The AGR soma is embedded in in connective tissue and can typically not be detected without desheathing.

In summary, RH795 staining allowed the comprehensive mapping of structural details never achieved in white light or dark-field views of intact STNS nerve tissue, demonstrating its ability to function as an anatomical marker. 
Desheathing the STG causes subtle changes in neuronal activity

The extraordinary access to neurons in the STG also allows testing the ability of RH795 for measuring membrane potential changes. The main reason for removing the ganglion sheath of the STG is that desheathing facilitates the recording of membrane potential changes with glass microelectrodes. Generally, the pyloric and gastric mill motor patterns are similar after desheathing to those generated without desheathing and to those generated in vivo (Hedrich et al., 2011; Diehl et al., 2013). However, quantitative comparisons are absent. It is understood that neuromodulators are released from descending neurons in the STG (Nusbaum and Beenhakker, 2002; Stein, 2009), and that extracellular peptidases limit the actions of these molecules and significantly affect neuronal output (Wood and Nusbaum, 2002). Desheathing may compromise this balanced system of peptidase activity and diffusion of neuromodulators and lead to changes in the response of neurons when neuromodulators are released. The anterior gastric receptor (AGR) neuron shows a conspicuous absence of response to neuromodulatory input, for example. AGR possesses neurites with postsynaptic structures in the STG neuropil (Goeritz et al., 2013), but reports about influences of STG motor activity and neuromodulator release on AGR are absent. Previous studies have failed to show any influence of pyloric, gastric mill, or descending neurons on the activity of AGR (Goeritz et al., 2013). We hypothesized that this conspicuous absence is due to the missing ganglion sheath in these experiments. Hence, we tested whether the presence of a sheath was influencing the activity of $A G R$, and found that desheathing can have subtle, but consistent influences on its firing patterns. Fig. 4A shows an extracellular recording of the activity of the sensory neuron AGR (Daur et al., 2009) 
during a gastric mill rhythm elicited by stimulation of the sensory nerve dpon ((Beenhakker et al., 2004), for details see Methods). AGR activity showed small fluctuations in firing frequency without desheathing that were timed with the gastric mill motor neurons (fig. 4B). On average, AGR instantaneous firing frequency (inst. ff.) was significantly higher during the activity phase of the lateral gastric motor neuron (LG; AGR inst. ff. $6.29 \pm 0.27 \mathrm{~Hz}, \mathrm{n}=20$ ) than during its functional antagonist, the dorsal gastric neuron (DG; AGR inst. ff. 5.88 $\pm 0.22 \mathrm{~Hz}, \mathrm{n}=20$; t-test, $p<0.001, n=20$ ). When we compared the change of AGR inst. ff. at different phases of the gastric mill rhythm, we found that the maximum occurred at phase 0.3 and the minimum at phase 0.7 , and that AGR inst. ff. was significantly different between these phases (t-test, $p<0.001, n=20$ ). After desheathing, however, AGR activity was tonic and no longer timed with the gastric mill rhythm (figs. 4A \& 4B, right). No significant differences were present between phases 0.3 and 0.7 (t-test, $n=20, p>0.6)$. In all animals tested $(N>10)$, desheathing abolished the oscillations in the AGR firing frequency.

RH795 does not alter neuronal activity in the STG We were curious whether $\mathrm{RH} 795$ would not only allow us to stain all neurons in the STG, but also would enable us to record membrane potential changes in the stained tissue using optical imaging without desheathing. As a prerequisite, we tested whether application of RH795 interfered with neuronal activity patterns. This was not the case, as also reported previously (Preuss and Stein, 2013): pyloric and gastric mill motor patterns could still be observed after staining the ganglion. Fig. 4B shows that even the rhythmic changes in AGR inst. ff. where still present after RH795. In both conditions, non-stained and stained (but non-desheathed), AGR's inst. ff. was significantly higher (t- 
test, $p<0.001, n=20$ ) during the activity phase of $L G$ (mean ff. $4.93 \pm 0.24 \mathrm{~Hz}, n=20$ ) than during the DG phase (fig. 4D, mean ff. $4.13 \pm 0.19 \mathrm{~Hz}, \mathrm{n}=20$ ). We also found a significant difference between phases 0.3 and 0.7 for both conditions (t-tests, $p<0.001$ STG nonstained; $p<0.01$ STG stained, $n=20$ ), which is consistent with our previous observations. In fact, there was no indication that staining the STG with RH795 had any influence on the AGR activity. A comparison of the mean values of all phases before and after staining shows no statistical difference (t-test, $p>0.8, n=20$ for each comparison). In summary, the gastric mill-timed oscillations in the AGR firing frequency were, unlike after desheathing (fig. 4A), still present after staining with RH795 (figs. 4C \& 4D), indicating that removing the ganglion sheath can in some instances change neuronal activity. The use of $\mathrm{RH} 795$ may circumvent these issues since it allows localizing the AGR soma (fig. 3D) without influencing AGR activity, and hence may allow AGR activity measurements without desheathing.

Neuronal activity can be imaged through the ganglion sheath

The neurons in the pyloric circuit in the STG are well-described (Stein, 2009), and their membrane potential oscillations and firing phases relative to other neurons and extracellularly recorded activity patterns are sufficient to unambiguously identify most neuron types participating in the gastric mill and pyloric rhythms. The pyloric rhythm is triphasic, has a cycle period of 0.5 - 2 seconds and can be monitored by recording the activities of the lateral pyloric (LP), pyloric dilator (PD) and pyloric constrictor (PY) neurons on the corresponding motor nerves ( $d v n$ or $/ v n$ for monitoring all three neuron types, pdn and pyn for exclusively monitoring the PDs and PYs, respectfully). 
To test whether $\mathrm{RH} 795$ permits optical recording of STG neuron activity through the ganglion sheath, we monitored the changes in fluorescence over time and compared them to the extracellularly recorded pyloric motor pattern without desheathing the STG. Fig. 5A shows a high-resolution photo of three adjacent STG neurons (20x objective) and the corresponding optical recordings from three selected regions of interest. The corresponding extracellular recording of the $p d n$ (bottom trace) shows the timing of the pyloric rhythm. All recordings were taken simultaneously (non-averaged data). While no consistent change in fluorescence was detected outside of neural structures (data not shown), the three neuronal somata showed fluorescence signals that correlated with the phasing of the pyloric motor neurons on the pdn (for visualization, see supplementary movie S1). Previously published measurements of the desheathed ganglion demonstrate that these changes in fluorescence represent the slow membrane potential oscillations of these neurons (Städele et al., 2012). Often, we were able to identify neurons solely based on their single-sweep optical signal (without averaging over multiple cycles) because the signal-to-noise ratio was high enough for a distinct comparison with the extracellular recording. For example, the top and bottom traces in fig. $5 \mathrm{~A}$ show a clear increase in fluorescence when the PD neurons were active, while the middle trace shows antiphasic activity that was in time with the LP neuron. Further analysis (cycle-triggered average, fig. 5B) revealed that the two PDtimed optical recordings indeed were PD neurons and that the LP-timed optical recording was the inferior cardiac (IC) neuron, which is active at approximately the same phase of the pyloric cycle as LP (Stein, 2009). For averaging, we used the simultaneously recorded activity of the PD neurons on the $p d n$ to determine the 
beginning and end of pyloric cycles, and then averaged across several pyloric cycles. Since averaging relies on the rhythmic activity of the motor neurons, this procedure facilitated neuron identification, and also revealed more of the neuronal subthreshold membrane potential changes, in particular of the PD neuron recording (since its action potentials were used to time the average). When we compared the frequency of the pyloric rhythm measured from the optical signal with that measured from the spike activity on the $p d n$, we found that they were virtually identical: Comparing the spectrograms of extracellular recording and optical signal (see Methods) revealed a high correlation in the frequency band that corresponded to the pyloric cycle frequency (fig. 5A, right) and there was a high correlation between the frequency components of the optical signals and the extracellular recording (top heat maps). All recordings showed a significant correlation ( $p<0.05, n=131$ for all comparisons) in the frequency domain. The highest correlation was found between the two PD neurons (Pearson product-moment correlation coefficients: IC and PD $1: 0.83 \pm 0.13, \mathrm{PD}_{2}$ and IC: $0.88 \pm$ 0.1, $\mathrm{PD}_{1}$ and $\mathrm{PD}_{2}: 0.91 \pm 0.09, \mathrm{IC}$ and $p d n: 0.65 \pm 0.1, \mathrm{PD}_{1}$ and $p d n: 0.68 \pm 0.07, \mathrm{PD}_{2}$ to pdn: $0.61 \pm 0.12$ ).

No individual action potentials could be detected in single-sweep recordings - a possible consequence of the slow sampling intervals of the camera (4 ms) and an undersampling of the fast membrane potential changes during the action potential. However, when we used spike-triggered averaging (a method typically used for identifying neurons in this system during intracellular recordings (Bucher et al., 2003)), we were able to measure synaptic interactions between neurons. PD receives inhibitory synaptic input from LP (Stein, 2009), and we have shown previously that synaptic 
potentials can be detected using optical recordings after spike-triggered averaging (Stein and Andras, 2010; Stein et al., 2011). Here, we triggered on the LP action potentials on the extracellular Ivn recording and simultaneously recorded PD fluorescence emission. Distinct IPSPs were obvious in the averaged signal of PD that occurred in response to the LP action potentials (fig. 5C), indicating that VSD imaging of synaptic events through the sheath is feasible and effective. The signal-to-noise ratio after averaging was high enough to also recognize the temporal synaptic dynamics of the LP-PD synapse. When we compared the averaged fluorescence change in PD that was elicited by the first LP action potential in the LP burst to that elicited by the last action potential, a clear decrease was obvious. This is due to synaptic depression present at this synapse (Manor et al., 1997).

For all optical recordings, we recognized a slow decrease in fluorescence intensity over time along with a diminution of the signal-to-noise ratio. Whether the reduction in fluorescence and signal quality was caused by bleaching or a slow washout of the dyes is unclear, but repetitive application of the dye was able to re-establish the staining and the signal-to-noise ratio (fig. 6). Continuous application thus allowed longlasting staining experiments.

Simultaneous recordings of multiple gastric mill motor neurons through the ganglion sheath

Because neurons in the STG are rather large $(10-100 \mu \mathrm{m}$ in diameter) and typically arranged in a single layer, they are easily accessible for intracellular recordings if the ganglion is desheathed. However, as we have shown above, desheathing can cause subtle changes in neural activities. RH795 might solve this dilemma by allowing us to 
localize and record most STG neurons simultaneously without the need for desheathing. The counterpoint to this would emphasize that the spike activity of most STG neurons can be recorded extracellularly. However, synaptic potentials and membrane potential changes cannot be observed. Also, not all STG neurons can be identified uniquely using extracellular recordings: one example is the two PD neurons shown in fig. 5A. There is no single nerve that contains individual PD axons for a separation of their activities. Yet, the PD neurons are strongly electrically coupled and usually they act in unison. For other neurons this is not clear. The 4 GM (gastric mill) neurons, for example, are weakly electrically coupled, but are active together since they receive similar synaptic input (Stein et al., 2005). Since their spikes on extracellular recordings are small and intermingled, they cannot reliably be separated on such recordings. As is the case for the PD neurons, there is no nerve that contains individual GM axons. In contrast to PD neurons, however, there are indications that the $4 \mathrm{GM}$ neurons have dissimilarities: The average number of GM axons on the $m v n$ is 2.95 (Daur et al., 2012) (and not 4), indicating that in any given animal, only a subset of GM neurons project through a given nerve and that, consequently, the GM neurons do not necessarily share the same axonal projection pathways. Here, we were using $\mathrm{RH} 795$ to simultaneously record the $4 \mathrm{GM}$ neurons to determine if they were active in unison, and if not, how conspicuous their differences were.

Fig. 7 shows a photo of the STG taken with the 10x objective after RH795 staining, and the corresponding optical recordings from 5 selected regions of interest. The 4 blue traces show single-sweep recordings of the $4 \mathrm{GMs}$. The yellow trace shows an optical recording of Interneuron 1 (Int1). Int1 is one of the smallest neurons in the 
STG and cannot be identified on extracellular recordings, since its action potential is buried among many others on the stn. Due to its small soma ( $5-10 \mu \mathrm{m}$ diameter), it also cannot be seen through the ganglion sheath, and even after desheathing, impalements are difficult. However, after RH795 staining, even small cell bodies such as that of Int1 were clearly visible without desheathing, and could be recorded optically. For comparing GM and Int1 activities, a gastric mill rhythm was elicited with dpon stimulation (see Methods). The corresponding extracellular recording of the Ign and dgn (bottom 2 traces) show the timing of the gastric mill rhythm after stimulation. All recordings were taken simultaneously with 20 ms sampling rate. The membrane potential changes of all 4 GM neurons, plus those of Int1 can easily be seen (visualized in supplemental movie S2). The GMs were active during the phase of the LG neuron, while Int1 was antiphasic to it, as previously described (Beenhakker et al., 2005). The correlation diagram (fig. 7, bottom) shows high synchrony between the 4 GM neurons, and antiphasic correlation between GMs and Int1. As indicated by the heat maps, while all maximum GM correlation factors were $>0.9$, slight variations existed when comparing each GM neuron in this condition. To confirm the identity of the neurons, after imaging we desheathed the ganglion and impaled the neurons with sharp microelectrodes and compared their spike activity and membrane potential oscillations to previously published data (Weimann et al., 1991; Beenhakker et al., 2005).

Our experiments thus show that using $\mathrm{RH} 795$, we can record and separate the activities of STG neurons that are inaccessible for other types of recordings without desheathing the ganglion. With respect to the $4 \mathrm{GM}$ neurons, within the limitation of the 
used imaging technique (frame rate and signal-to-noise ratio), we found a high synchrony amongst the GM neurons.

\section{Discussion}

We demonstrate that when bath-applied to neural tissue, $\mathrm{RH} 795$ permeates neural sheaths and stains sub-sheath neuronal compartments, indicating its potential as an anatomical marker without desheathing. In addition, we successfully recorded neural activity through the sheath via changes in fluorescence of neuronal membrane-bound $\mathrm{RH} 795$. These findings suggest the use of optical imaging with $\mathrm{RH} 795$ as an alternative to protocols that require the removal of the sheath prior to experimentation.

RH795 as an anatomical marker for living neural tissue

Discriminating between individual neurons is essential in any attempt to study neural networks and their function—a task particularly difficult in living tissue and during the recording of neural activities. Most recent studies thus now comprise combinations of several techniques that allow staining the neural tissue and the simultaneous measurement of activity (Zhong et al., 2010; Ampatzis et al., 2013). Identifying cellular structures such as somata and axons is particularly important in light of current efforts to decipher the connectome of neural networks in many systems (Sporns et al., 2005; Sporns, 2011; Bargmann and Marder, 2013). In the simplest case, activity measurements are performed without detailed visual guidance ('blind recordings') and only afterwards, neural structures are then identified (using retrograde labeling, chemical precipitation or stereotactic placement of the electrodes, for example). For single cell recording with microelectrodes, intracellular injection of (fluorescent) dyes can label the recorded neurons during the experiment, and allows the analysis of the 
associated neuritic arborizations after the experiments, producing an anatomical layout of the recorded cells (Wouterlood et al., 1990). In any of these cases, the success of the experiment usually depends on the experience of the experimenter in localizing the neurons in question. More recently, the use of fluorescence techniques has become an aid in determining the anatomical structure of otherwise unidentifiable neuronal features (Grinvald and Hildesheim, 2004; Fenno et al., 2011). While retrograde backfilling of axons with fluorescent dyes allows the staining of distinct neurons even before an experiment, the expression of fluorescent proteins such as GFP, calcium- or voltageindicators in selected neural tissues has facilitated neuronal identification in living tissue (Aramaki and Hatta, 2006; Ferezou et al., 2006; Lichtman and Denk, 2011; Kim and Jun, 2013). Targeted expression of fluorescent proteins now provides a tool for guiding electrode placement and anatomical descriptions of distinct neural tissue even in large brains. Since expression is targeted to single neuron types, several driver lines are needed to dissect whole circuits, as these consist of many neuron types. Targeted expression is thus equivalent to the identification of individual neurons in smaller nervous systems. Using Brainbow, a protein translation of fluorescent protein variants to yield a vast array of coloring, even individual cells of the same type can be visually separated (Livet et al., 2007; Lichtman et al., 2008; Pan et al., 2011), albeit with the disadvantage that each color combination is unique for each animal. All expression techniques are reserved for genetically well-characterized organisms with appropriate driver lines, and are thus not available for many classical model systems with already defined anatomical and functional connectivity. In addition, due to its specificity, genetic 
expression only ever targets subsets of neural circuits, and visualizing other circuit neurons depends on the availability of appropriate driver lines.

As an alternative, bath- and focal-applications, which are routinely used for drug and modulator administration, can be employed to deliver fluorescent dyes for bulk staining of cells (Salzberg et al., 1977; Cohen et al., 1978). Various dyes are available that stain different cellular structures. While DAPI, for example, stains nuclear material in living tissue (Kapuscinski, 1995), exogenous applications of VSDs and calciumsensitive dyes stain cell membranes and cytoplasm, respectively. While fluorometric calcium imaging requires membrane-permeant acetoxymethyl (AM) ester forms of the dye, many VSD associate with the neuronal membrane, revealing the outline of the stained neurons. Only cells within the application site will be stained, but within this area, the staining will lack specificity. One of the drawbacks of these dyes is that, for unknown reasons, not all dyes work in all systems (Chemla and Chavane, 2010; Lim et al., 2012). Potentially, chemical or mechanical differences between systems prevent the dyes from reaching the neural tissue. In particular in small invertebrate preparations, dyes have to either penetrate a nerve sheath in the periphery, or a ganglion sheath in more central regions of the nervous system. Often sheaths, glia cells and connective tissue impede dye penetration and must be removed surgically or enzymatically. During this potentially dangerous process, one risks mechanically damaging the nervous system while performing a microsurgery, along with more subtle effects related to changes in neural activity. We show that oscillations in the firing frequency of the proprioceptive neuron AGR in the STG disappear once the ganglion is desheathed, although all other features of neural activity remained intact. Thus, removing the sheath 
had subtle effects on the behavior of the system, potentially making the information acquired from such experiments less reliable. While the contribution of the ganglion sheath to neural activity is unclear in most systems, there are indications in several systems that removing the sheath might interfere with ionic balance (Dörr et al., 1996) and mechanical properties (Debrodt and Bässler, 1989). We show that bath-application of the fluorescent VSD RH795 enhanced visibility of neural structures from the prestained condition in ganglia of three different species across two phyla without the need for removing the sheath. $\mathrm{RH} 795$ reliably stained somata, neurites and axons. In all ganglia cell bodies in several different layers of the ganglion could be located, despite the three dimensional structure of most ganglia. In the STG, which comprises only a single to a few layers of neurons, $\mathrm{RH} 795$ revealed many individually identifiable axons and neurites in a clarity never reported before in living tissue. In particular at the anterior end of the STG where axons were spatially spread out, individual axons of modulatory projection neurons that enter the STG (Coleman et al., 1992) could be separated, in addition to identified motor neurons such as CD1. Intracellular recordings from these axons are traditionally performed in dark-field illumination (Bartos and Nusbaum, 1997), but our comparison of dark-field and fluorescence staining clearly reveals the superiority of the $\mathrm{RH} 795$ staining. It is thus conceivable that visually locating distinct axons before impalement will significantly increase recording success. Furthermore, our staining revealed axons that lay on top of cell bodies of other neurons, potentially impeding access to these neurons. 
More importantly, RH795 stained axons and cell bodies in peripheral nerves through the sheath. In recent years, the modulation of axonal action potential propagation has attracted a lot of attention, demonstrating that axons are not mere cables that faithfully conduct information (Bucher and Goaillard, 2011). Rather, the intrinsic properties of peripheral axons are modified by neuromodulators via metabotropic receptors in the axon membrane. Axonal recordings are experimentally difficult and locating axons in the nerve is a major concern when multiple locations along the axon need to be recorded. Even in small invertebrates, motor and sensory axons are several centimeters long, enwrapped in a nerve sheath and packed into axon bundles (Coleman et al., 1992). Traditional methods for axon stainings, such as intracellular injection of dyes, only work at short distances, since diffusion of the dye inside of the neuron reduces dye concentration with distance. $\mathrm{RH} 795$ reliably stained axons through the nerve sheath without noteworthy residual staining of the sheath and revealed the fine details of individual axons and varicosities even in nerves with many (>60) axons. In nerves with fewer axons, almost all axons were immediately visible. For example, the axons of the two PD neurons, which are modulated by dopamine (Ballo et al., 2010), were obvious in stainings of the $p d n$. In the small sensorimotor nerve gpn, we were able to locate the cell body of the gastro-pyloric receptor neuron, which has so far only been possible with retrograde labeling (Katz et al., 1989). Furthermore, we were able to successfully localize AGR with $\mathrm{RH} 795$ staining through the sheath. We have shown that AGR activity changes when desheathing, so $\mathrm{RH} 795$ stainings will be particularly helpful in future experiments for locating the AGR soma for intracellular or optical recordings through the sheath. 
In summary, RH795 staining allowed the comprehensive mapping of structural details in the STG never achieved in intact nerve tissue. Hence, RH795 is a good anatomical marker for determining cell body location as well as neuritic organization in living tissue.

RH795 can be used to monitor neuronal activity through nerve sheaths To understand neural circuit function, the detailed characterization of the activity of many, if not all, circuit neurons is needed (Briggman and Denk, 2006; Hill et al., 2012; Bargmann and Marder, 2013), and ideally the location of the recorded neurons should be determined before or during the recording attempt. $\mathrm{RH} 795$ not only revealed anatomical features not observable in a non-stained ganglion, but also allowed us to record membrane potential changes through the sheath. While removing the sheath also enhances visibility in non-stained ganglia and facilitates access to neurons with microelectrodes, it comes with the caveats of possible damage to cells and activity. Most VSDs and calcium AM dyes are applied to desheathed ganglia (Briggman and Kristan Jr., 2006; Obaid and Salzberg, 2009; Stein et al., 2011; Hill et al., 2012) and, in the STG, do not appear to permeate the sheath (data not shown). We demonstrate that staining neurons through the sheath with $\mathrm{RH} 795$ not only retained the fine details of the neuronal activity, but also allowed us to record multiple neurons simultaneously and identify them using single-sweep (non-averaged) recordings. After spike-triggered averaging, the signal-to-noise ratio was high enough to detect the temporal synaptic dynamics of IPSPs between the LP and PD neurons. In general, the signal-to-noise ratio of optical imaging is lower than for direct measurements of the membrane potential with glass microelectrodes, although there have been significant improvements in the 
last decade (Salzberg et al., 1977; Siegel and Isacoff, 1997; Obaid et al., 2004;

Fromherz et al., 2008; Miller et al., 2012; Cao et al., 2013). There are more sources of optical noise than electrical (e.g. shot noise, dark noise and extraneous noise,(Salzberg, 1983; Zochowski et al., 2000)). However, by averaging over multiple cycles, most of the noise can be removed.

Toxicity of the dyes is also an issue that needs to be taken into account. Most VSDs cause toxic effects over time, or affect neuronal properties that alter activity (Mennerick et al., 2010). We have previously shown that $\mathrm{RH} 795$ has no discernable effect on the STG motor patterns and no apparent toxic influence when applied. Prolonged illumination, however, can have deleterious effects on the motor pattern, limiting the duration of the imaging session (Stein and Andras, 2010; Preuss and Stein, 2013). We also recognized a slow diminution of the staining over the course of several hours and along with it a diminishing signal-to-noise ratio. This effect was reversible by additional RH795 applications. Most importantly, RH795 allowed us to accurately monitor both the activity of fast pyloric and slow gastric mill neurons over an extended period of time. For the latter, we demonstrate the simultaneous recording of $4 \mathrm{GM}$ neurons and Int1. We chose these particular neurons since their recording demonstrates the advantages of optical imaging over other recording techniques when it comes to separating the activities of neurons of the same type (such as the GMs) or recoding from small interneurons. The GMs are particularly interesting: they innervate the gm1 muscle (Selverston and Moulins, 1987), and intracellular recordings from individual GMs revealed similar synaptic input in all GMs (Stein et al., 2005). Yet, GM neurons appear to vary in the projection pattern of their axons: The average number of 
GM axons on the $m v n$ is 2.95 , indicating that subpopulations of GM neurons with different axonal projection patterns and possibly distinct activity patterns may exist. Nevertheless, our results indicate that, within the limitations of the optical imaging, GM membrane potentials were highly correlated during a VCN-elicited gastric mill rhythm (Beenhakker et al., 2004; Diehl et al., 2013).

\section{Functional implications}

Int1 and GM neurons are part of the gastric mill CPG (Stein et al., 2007) whose activity pattern is influenced by indirect feedback from the aforementioned proprioceptive neuron AGR. Our findings support the conclusion of Daur et al. (2009) in that axonal modulation not only occurs spontaneously, but is reliably present when gastric mill rhythms are elicited. While changes in AGR firing frequency had been observed, they had never been correlated to the motor pattern before. We have previously shown that such small changes in AGR's spontaneous activity have significant effects on the motor pattern produced, as they determine the state of the neuromodulatory system that drives the gastric mill rhythm (Daur et al., 2009). Thus, as AGR loses its oscillatory activity after desheathing, it is particularly important to keep the sheath intact while recording from Int1 and the GM neurons, which for all intents and purposes prevents multiple intracellular recordings. Extracellular recordings from motor nerves, on the other hand, cannot reveal Int1 activity, and do not allow separation of the 4 GMs from one another, a task easily solved by optical imaging with $\mathrm{RH} 795$.

With respect to AGR, our findings explain a previously published, conspicuous finding, namely that AGR activity appeared to be unaffected by other STG neurons despite the fact that it possesses putative postsynaptic structures on neurites in the 
STG (Goeritz et al., 2013). In these experiments, desheathing the ganglion was necessary to locate AGR and its axons, but apparently reduced or abolished the postsynaptic response of AGR. We show that such a response is present in the nondesheathed ganglion and that applying $\mathrm{RH} 795$ does not influence the AGR response. Moreover, RH795 allows the localization of the AGR cell body without desheathing.

Because of the dual character of $\mathrm{RH} 795$ to function as an anatomical marker and as voltage indicator of membrane potential changes, there are multiple applications of this technique in addition to the examples demonstrated in this paper. In general, studies using semi-intact preparations may benefit from this approach as it can be difficult to maintain intracellular somatic recordings from neurons while the musculature is present and allowed to move. Depending on the species used, it may also be difficult to desheath ganglia in semi-intact preparations, given that nerves and connectives must remain intact.

This technique can also be applied to other ganglia in the STNS, like the commissural ganglia, which contains neurons that modulate the activity of STG neurons (Nusbaum and Beenhakker, 2002; Stein, 2009) and are much less characterized. In particular for preliminary studies, using $\mathrm{RH} 795$ may be an expeditious technique to acquire information about populations of modulatory cells, and to characterize the response and location of these neurons.

In summary, $\mathrm{RH} 795$ provides a relatively unique tool for recording many neurons simultaneously in living tissues without the need for removing neural sheaths, and has a plethora of other potential applications not yet investigated. 
Used with permission from Goldsmith CJ, Städele C, Stein W. 2014. Optical imaging of fine neural structures in non-desheathed nervous systems. PLoS One. 9(7): e103459. CC BY 4.0.

Author Contributions: conceived and designed the experiments: CG CS WS. Performed the experiments: CG CS WS. Analyzed the data: CG CS WS. Wrote the paper: CG CS WS. 


\section{REFERENCES}

Ampatzis K, Song J, Ausborn J, El Manira A. 2013. Pattern of Innervation and Recruitment of Different Classes of Motoneurons in Adult Zebrafish. J Neurosci $33: 10875-10886$.

Aramaki S, Hatta K. 2006. Visualizing neurons one-by-one in vivo: Optical dissection and reconstruction of neural networks with reversible fluorescent proteins. Dev Dyn 235:2192-2199.

Baker BJ, Kosmidis EK, Vucinic D, Falk CX, Cohen LB, Djurisic M, Zecevic D. 2005. Imaging brain activity with voltage- and calcium-sensitive dyes. Cell Mol Neurobiol 25:245-282.

Ballo AW, Keene JC, Troy PJ, Goeritz ML, Nadim F, Bucher D. 2010. Dopamine Modulates Ih in a Motor Axon. J Neurosci 30:8425-8434.

Bargmann Cl, Marder E. 2013. From the connectome to brain function. Nat Methods 10:483-490.

Bartos M, Nusbaum MP. 1997. Intercircuit control of motor pattern modulation by presynaptic inhibition. J Neurosci 17:2247-2256.

Beenhakker MP, Blitz DM, Nusbaum MP. 2004. Long-lasting activation of rhythmic neuronal activity by a novel mechanosensory system in the crustacean stomatogastric nervous system. J Neurophysiol 91:78-91.

Beenhakker MP, DeLong ND, Saideman SR, Nadim F, Nusbaum MP. 2005.

Proprioceptor regulation of motor circuit activity by presynaptic inhibition of a modulatory projection neuron. J Neurosci 25:8794-8806. 
Blitz DM, Nusbaum MP. 2011. Neural circuit flexibility in a small sensorimotor system.

Curr Opin Neurobiol 21:544-552.

Briggman KL, Abarbanel HD, Kristan Jr. WB. 2005. Optical imaging of neuronal populations during decision-making. Science (80- ) 307:896-901.

Briggman KL, Abarbanel HD, Kristan Jr. WB. 2006. From crawling to cognition: analyzing the dynamical interactions among populations of neurons. Curr Opin Neurobiol 16:135-144.

Briggman KL, Denk W. 2006. Towards neural circuit reconstruction with volume electron microscopy techniques. Curr Opin Neurobiol 16:562-570.

Briggman KL, Kristan Jr. WB. 2006. Imaging dedicated and multifunctional neural circuits generating distinct behaviors. J Neurosci 26:10925-10933.

Brown TG. 1911. The intrinsic factors in the act of progression in the mammal. Proc $R$ Soc London Ser B, Contain Pap a Biol Character 84:308-319.

Bucher D, Goaillard J-M. 2011. Beyond faithful conduction: short-term dynamics, neuromodulation, and long-term regulation of spike propagation in the axon. Prog Neurobiol 94:307-346.

Bucher D, Johnson CD, Marder E. 2007. Neuronal morphology and neuropil structure in the stomatogastric ganglion of the lobster, Homarus americanus. J Comp Neurol 501:185-205.

Bucher D, Thirumalai V, Marder E. 2003. Axonal dopamine receptors activate peripheral spike initiation in a stomatogastric motor neuron. J Neurosci 23:6866-6875.

Cao G, Platisa J, Pieribone VA, Raccuglia D, Kunst M, Nitabach MN. 2013. Genetically targeted optical electrophysiology in intact neural circuits. Cell 154:904-913. 
Chemla S, Chavane F. 2010. Voltage-sensitive dye imaging: Technique review and models. J Physiol Paris 104:40-50.

Cohen LB, Salzberg BM. 1978. Optical measurement of membrane potential. Rev Physiol Biochem Pharmacol 83:35-88.

Cohen LB, Salzberg BM, Grinvald A. 1978. Optical methods for monitoring neuron activity. Annu Rev Neurosci 1:171-182.

Coleman MJ, Nusbaum MP, Cournil I, Claiborne BJ. 1992. Distribution of modulatory inputs to the stomatogastric ganglion of the crab, Cancer borealis. J Comp Neurol $325: 581-594$.

Daur N, Diehl F, Mader W, Stein W. 2012. The stomatogastric nervous system as a model for studying sensorimotor interactions in real-time closed-loop conditions. Front Comput Neurosci 6.

Daur N, Nadim F, Stein W. 2009. Regulation of motor patterns by the central spikeinitiation zone of a sensory neuron. Eur J Neurosci 30:808-822.

Debrodt B, Bässler U. 1989. Motor neurones of the flexor tibiae muscle in phasmids. Zool Jahrbücher Abteilung für Allg Zool und Physiol der Tiere 93:481-494.

Dickinson PS, Marder E. 1989. Peptidergic modulation of a multioscillator system in the lobster. I. Activation of the cardiac sac motor pattern by the neuropeptides proctolin and red pigment-concentrating hormone. J Neurophysiol 61:833-844.

Diehl F, White RS, Stein W, Nusbaum MP. 2013. Motor circuit-specific burst patterns drive different muscle and behavior patterns. J Neurosci 33:12013-12029. 
Dörr H, Heß D, Gramoll S. 1996. Interstitial voltage and potassium concentration in the mesothoracic ganglion of a stick insect at rest and during neuronal activation. $\mathrm{J}$ Insect Physiol 42:967-974.

Dubuc R, Brocard F, Antri M, Fenelon K, Gariepy JF, Smetana R, Menard A, Le Ray D, Viana Di Prisco G, Pearlstein E, Sirota MG, Derjean D, St-Pierre M, Zielinski B, Auclair F, Veilleux D. 2008. Initiation of locomotion in lampreys. Brain Res Rev $57: 172-182$.

Fenno L, Yizhar O, Deisseroth K. 2011. The development and application of optogenetics. Annu Rev Neurosci 34:389-412.

Ferezou I, Bolea S, Petersen CCH. 2006. Visualizing the cortical representation of whisker touch: voltage-sensitive dye imaging in freely moving mice. Neuron $50: 617-629$.

Fromherz P, Hübener G, Kuhn B, Hinner MJ. 2008. ANNINE-6plus, a voltage-sensitive dye with good solubility, strong membrane binding and high sensitivity. Eur Biophys J 37:509-514.

Galizia CG, Joerges J, Kättner A, Faber T, Menzel R. 1997. A semi-in-vivo preparation for optical recording of the insect brain. J Neurosci Methods 76:61-69.

Goeritz ML, Bowers MR, Slepian B, Marder E. 2013. Neuropilar projections of the anterior gastric receptor neuron in the stomatogastric ganglion of the Jonah crab, Cancer borealis. PLoS One 8:e79306.

Grillner S, Ekeberg Ö, El Manira A, Lansner A, Parker D, Tegnér J, Wallén P. 1998. Intrinsic function of a neuronal network - a vertebrate central pattern generator. Brain Res Rev 26:184-197. 
Grinvald A, Hildesheim R. 2004. VSDI: a new era in functional imaging of cortical dynamics. Nat Rev Neurosci 5:874-885.

Grinvald A, Lieke EE, Frostig RD, Hildesheim R. 1994. Cortical point-spread function and long-range lateral interactions revealed by real-time optical imaging of macaque monkey primary visual cortex. J Neurosci 14:2545-2568.

Gutierrez GJ, Grashow RG. 2009. Cancer borealis stomatogastric nervous system dissection. J Vis Exp 25:e1207.

Harris-Warrick RM. 2011. Neuromodulation and flexibility in Central Pattern Generator networks. Curr Opin Neurobiol 21:685-692.

Hedrich UB, Diehl F, Stein W. 2011. Gastric and pyloric motor pattern control by a modulatory projection neuron in the intact crab Cancer pagurus. J Neurophysiol 105:1671-1680.

Hedrich UB, Smarandache CR, Stein W. 2009. Differential activation of projection neurons by two sensory pathways contributes to motor pattern selection. $\mathrm{J}$ Neurophysiol 102:2866-2879.

Hill ES, Vasireddi SK, Bruno AM, Wang J, Frost WN. 2012. Variable Neuronal Participation in Stereotypic Motor Programs. PLoS One 7:e40579.

Insausti TC, Lazzari CR, Casas J. 2011. The morphology and fine structure of the giant interneurons of the wood cricket Nemobius sylvestris. Tissue Cell 43:52.

Kapuscinski J. 1995. DAPI: a DNA-specific fluorescent probe. Biotech Histochem $70: 220-233$. 
Katz PS, Eigg MH, Harris-Warrick RM. 1989. Serotonergic/cholinergic muscle receptor cells in the crab stomatogastric nervous system. I. Identification and characterization of the gastropyloric receptor cells. J Neurophysiol 62:558-570.

Katz PS, Harris-Warrick RM. 1991. Recruitment of crab gastric mill neurons into the pyloric motor pattern by mechanosensory afferent stimulation. J Neurophysiol 65:1442-1451.

Kim SA, Jun SB. 2013. In-vivo Optical Measurement of Neural Activity in the Brain. Exp Neurobiol 22:158-166.

Kristan Jr. WB, Calabrese RL, Friesen WO. 2005. Neuronal control of leech behavior. Prog Neurobiol 76:279-327.

Lichtman JW, Denk W. 2011. The big and the small: challenges of imaging the brain's circuits. Science (80- ) 334:618-623.

Lichtman JW, Livet J, Sanes JR. 2008. A technicolour approach to the connectome. Nat Rev Neurosci 9:417-422.

Lim DH, Mohajerani MH, LeDue J, Boyd J, Chen S, Murphy TH. 2012. In vivo largescale cortical mapping using channelrhodopsin-2 stimulation in transgenic mice reveals asymmetric and reciprocal relationships between cortical areas. Front Neural Circuits 6.

Livet J, Weissman TA, Kang H, Lu J, Bennis RA, Sanes JR, Lichtman JW. 2007. Transgenic strategies for combinatorial expression of fluorescent proteins in the nervous system. Nature 450:56-62.

El Manira A, Shenoy K. 2009. Motor systems. Curr Opin Neurobiol 19:570-571. 
Manor Y, Nadim F, Abbott LF, Marder E. 1997. Temporal dynamics of graded synaptic transmission in the lobster stomatogastric ganglion. J Neurosci 17:5610-5621.

Marder E, Bucher D. 2007. Understanding circuit dynamics using the stomatogastric nervous system of lobsters and crabs. Annu Rev Physiol 69:291-316.

Mennerick S, Chisari M, Shu HJ, Taylor A, Vasek M, Eisenman LN, Zorumski CF. 2010. Diverse voltage-sensitive dyes modulate GABAA receptor function. J Neurosci 30:2871-2879.

Miller EW, Lin JY, Frady EP, Steinbach PA, Kristan WB, Tsien RY. 2012. Optically monitoring voltage in neurons by photo-induced electron transfer through molecular wires. Proc Natl Acad Sci 109:2114-2119.

Nusbaum MP, Beenhakker MP. 2002. A small-systems approach to motor pattern generation. Nature 417:343-350.

Obaid AL, Loew LM, Wuskell JP, Salzberg BM. 2004. Novel naphthylstyryl-pyridium potentiometric dyes offer advantages for neural network analysis. J Neurosci Methods 134:179-190.

Obaid AL, Salzberg BM. 2009. Optical recording of electrical activity in guinea-pig enteric networks using voltage-sensitive dyes. J Vis Exp.

Pan YA, Livet J, Sanes JR, Lichtman JW, Schier AF. 2011. Multicolor Brainbow imaging in zebrafish. Cold Spring Harb Protoc 2011:pdb. prot5546.

Preuss S, Stein W. 2013. Comparison of two voltage-sensitive dyes and their suitability for long-term imaging of neuronal activity. PLoS One 8:e75678.

Rossignol S, Dubuc R, Gossard JP. 2006. Dynamic sensorimotor interactions in locomotion. Physiol Rev 86:89-154. 
Salzberg BM. 1983. Optical recording of electrical activity in neurons using molecular probes. In: Barker J, McKelvy J, editors. Current Methods in Cellular Neurobiology. New York: John Wiley and Sons, Inc. p 139-187.

Salzberg BM, Davila H V, Cohen LB. 1973. Optical recording of impulses in individual neurones of an invertebrate central nervous system. Nature 246:508-509.

Salzberg BM, Grinvald A, Cohen LB, Davila H V, Ross WN. 1977. Optical recording of neuronal activity in an invertebrate central nervous system: simultaneous monitoring of several neurons. J Neurophysiol 40:1281-1291.

Selverston Al, Moulins M. 1987. The crustacean stomatogastric system: a model for the study of central nervous systems. Springer-Verlag Heidelberg.

Siegel MS, Isacoff EY. 1997. A genetically encoded optical probe of membrane voltage. Neuron 19:735-741.

Smarandache CR, Stein W. 2007. Sensory-induced modification of two motor patterns in the crab, Cancer pagurus. J Exp Biol 210:2912-2922.

Sporns O. 2011. The human connectome: a complex network. Ann N Y Acad Sci 1224:109-125.

Sporns O, Tononi G, Kötter R. 2005. The human connectome: a structural description of the human brain. PLoS Comput Biol 1:e42.

Städele C, Andras P, Stein W. 2012. Simultaneous measurement of membrane potential changes in multiple pattern generating neurons using voltage sensitive dye imaging. J Neurosci Methods 203:78-88.

Stein W. 2009. Modulation of stomatogastric rhythms. J Comp Physiol A Neuroethol Sens Neural Behav Physiol 195:989-1009. 
Stein W, Andras P. 2010. Light-induced effects of a fluorescent voltage-sensitive dye on neuronal activity in the crab stomatogastric ganglion. J Neurosci Methods 188:290294.

Stein W, DeLong ND, Wood DE, Nusbaum MP. 2007. Divergent co-transmitter actions underlie motor pattern activation by a modulatory projection neuron. Eur $\mathrm{J}$ Neurosci 26:1148-1165.

Stein W, Eberle CC, Hedrich UBS. 2005. Motor pattern selection by nitric oxide in the stomatogastric nervous system of the crab. Eur J Neurosci 21:2767-2781.

Stein W, Städele C, Andras P. 2011. Single-sweep voltage-sensitive dye imaging of interacting identified neurons. J Neurosci Methods 194:224-234.

Telkes I, Csoknya M, Buzás P, Gábriel R, Hámori J, Elekes K. 1996. GABAimmunoreactive neurons in the central and peripheral nervous system of the earthworm, Lumbricus terrestris (Oligochaeta, Annelida). Cell Tissue Res 285:463475.

Vedel JP, Moulins M. 1977. A motor neuron involved in two centrally generated motor patterns by means of two different spike initiating sites. Brain Res 138:347-352.

Wang Y, Jing G, Perry S, Bartoli F, Tatic-Lucic S. 2009. Spectral characterization of the voltage-sensitive dye di-4-ANEPPDHQ applied to probing live primary and immortalized neurons. Opt Express 17:984-990.

Weimann JM, Meyrand P, Marder E. 1991. Neurons that form multiple pattern generators: identification and multiple activity patterns of gastric/pyloric neurons in the crab stomatogastric system. J Neurophysiol 65:111-122.

Williamson R, Chrachri A. 2004. Cephalopod neural networks. Neurosignals 13:87-98. 
Wood DE, Nusbaum MP. 2002. Extracellular peptidase activity tunes motor pattern modulation. J Neurosci 22:4185-4195.

Wouterlood FG, Jorritsma-Byham B, Goede PH. 1990. Combination of anterograde tracing with Phaseolus vulgaris -leucoagglutinin, retrograde fluorescent tracing and fixed-slice intracellular injection of Lucifer yellow. J Neurosci Methods 33:207-217.

Zhong G, Droho S, Crone SA, Dietz S, Kwan AC, Webb WW, Sharma K, Harris-Warrick RM. 2010. Electrophysiological characterization of V2a interneurons and their locomotor-related activity in the neonatal mouse spinal cord. J Neurosci 30:170182.

Zochowski M, Wachowiak M, Falk CX, Cohen LB, Lam YW, Antic S, Zecevic D. 2000. Imaging membrane potential with voltage-sensitive dyes. Biol Bull 198:1-21. 


\section{FIGURES}

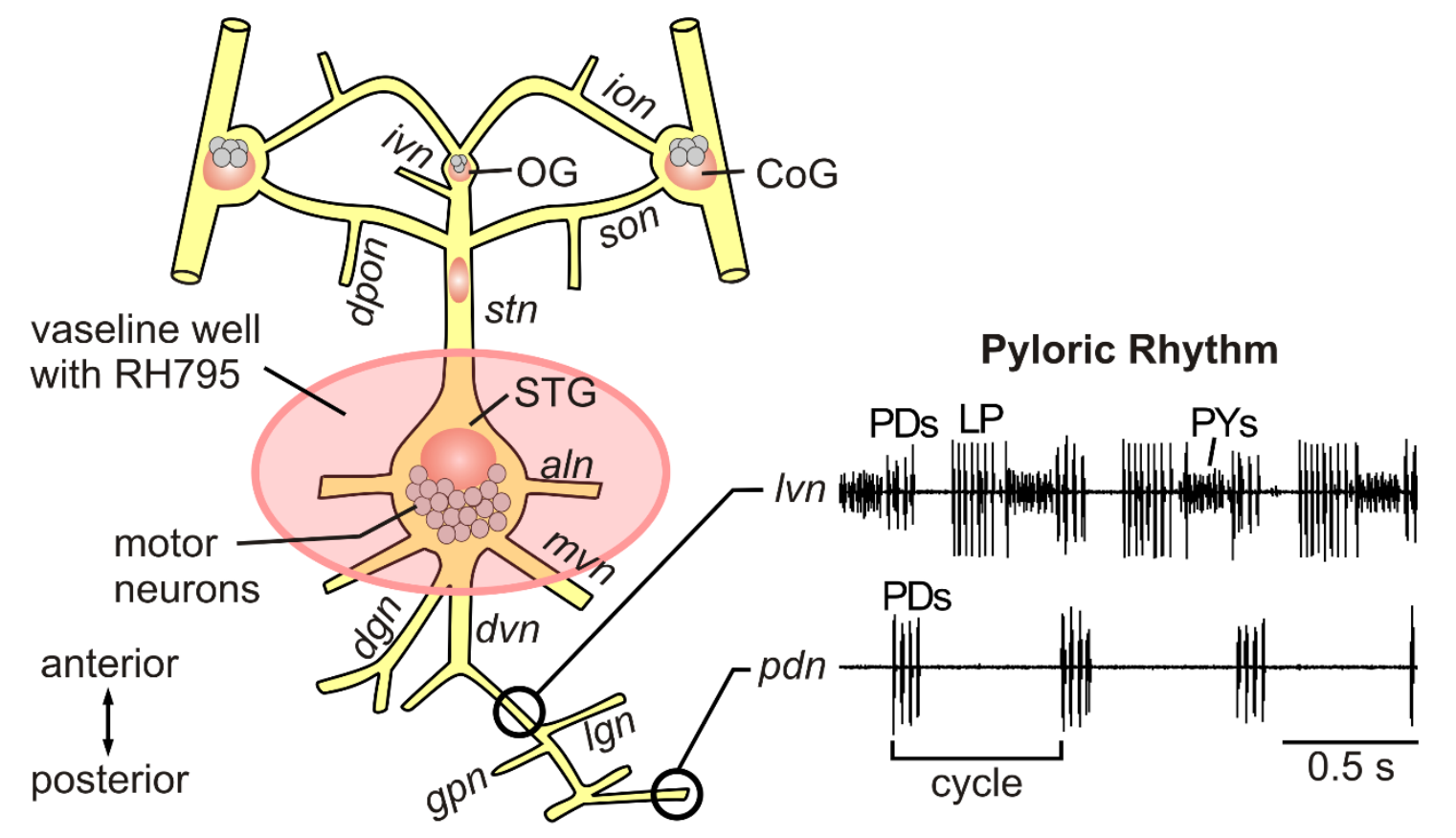

Fig. 1. Schematic overview of the stomatogastric nervous system (STNS) of the crab, Cancer borealis. The pink circle marks the application site for the VSD RH795. The black circles illustrate two different extracellular recording sites and the corresponding neuronal signals. The three main neuron types (PD, LP, PY) participating in the pyloric rhythm can be monitored on the IVn. The pdn selectively shows the activity of the two PD neurons. Grey cell bodies illustrate neurons in the stomatogastric ganglion (STG), the oesophageal ganglion $(\mathrm{OG})$ and the commissural ganglia $(\mathrm{CoG})$. Neurons: PD, pyloric dilator neuron; LP, lateral pyloric neuron; PY, pyloric constrictor neuron. Nerves: ivn, inferior ventricular nerve; ion, inferior esophageal nerve; son, superior esophageal nerve; dpon, dorsal posterior oesophageal nerve; stn, stomatogastric nerve; aln, anterior lateral nerve; $m v n$, median ventricular nerve; dgn, dorsal gastric nerve; $d v n$, dorsal ventricular nerve; Ivn, lateral ventricular nerve; gpn, gastro pyloric nerve; Ign, lateral gastric nerve; $p d n$, pyloric dilator nerve. 


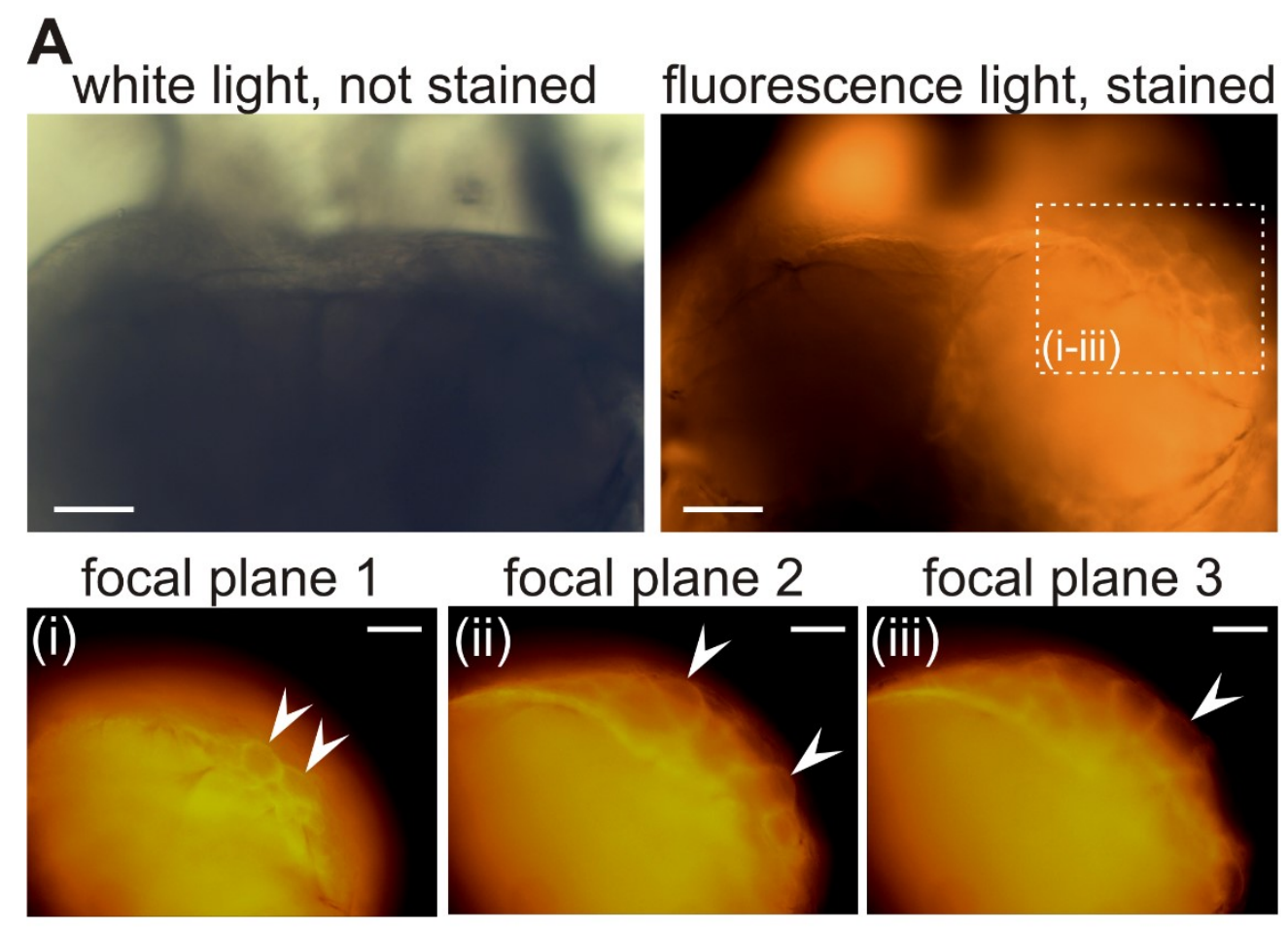

B

white light, not stained

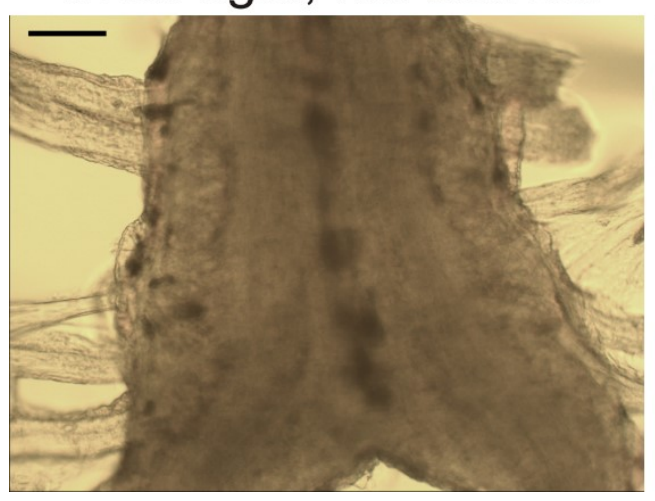

fluorescence light, stained

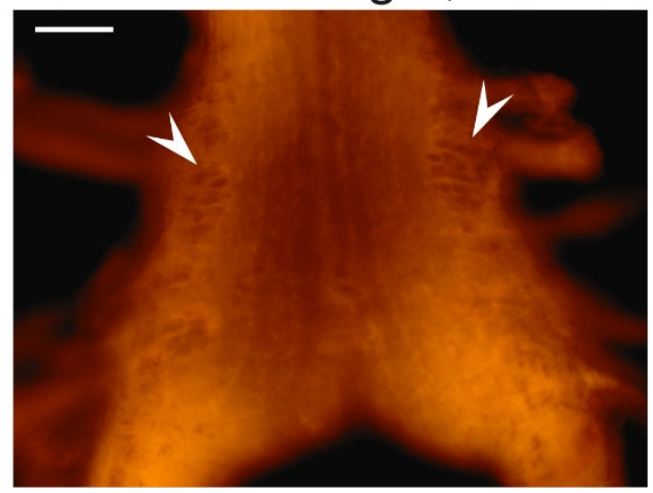

Fig. 2. RH795 stains through the ganglion sheath and allows the visualization of cell body location. A: Metathoracic ganglion of the cricket $G$. sigillatus before (white light) and after staining (fluorescence light). Scale bar is $100 \mu \mathrm{m}$. (i-iii) Higher magnification of the dotted area in the picture above. Changing the focal plane from dorsal (i) to ventral (iii) revealed multiple layers of distinct neurons (see arrows). Scale bar is $50 \mu \mathrm{m}$. B: Subpharyngeal ganglion of the earthworm $E$. hortensis before and after staining with $\mathrm{RH} 795$. Large neuronal somata were visible after staining (see arrows). Scale bar is $100 \mu \mathrm{m}$. 
A Cell visibility before (I) and after STG desheathing (II)

(i)

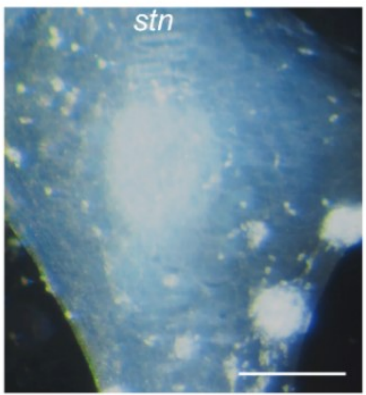

B Visibility change during washout of RH795

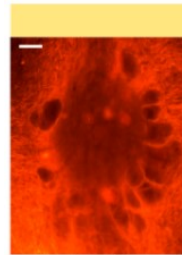

$1 \mathrm{~min}$

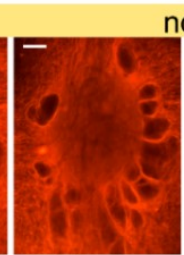

$20 \mathrm{~min}$ non-desheathed

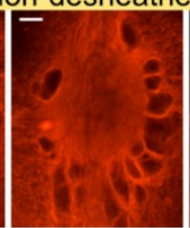

$60 \mathrm{~min}$

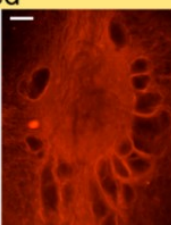

$120 \mathrm{~min}$

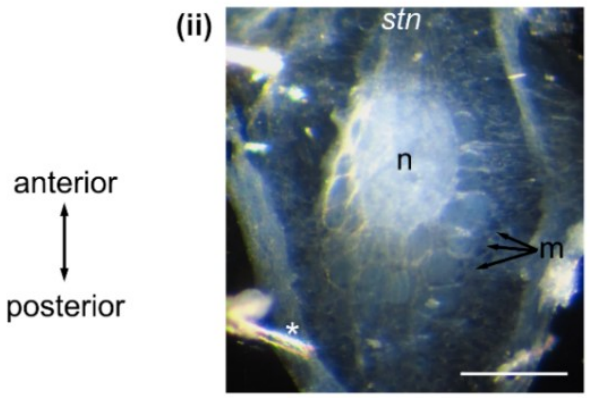

(i)

desheathed

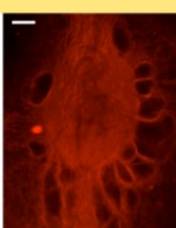

$180 \mathrm{~min}$

post washout

C Axonal stainings
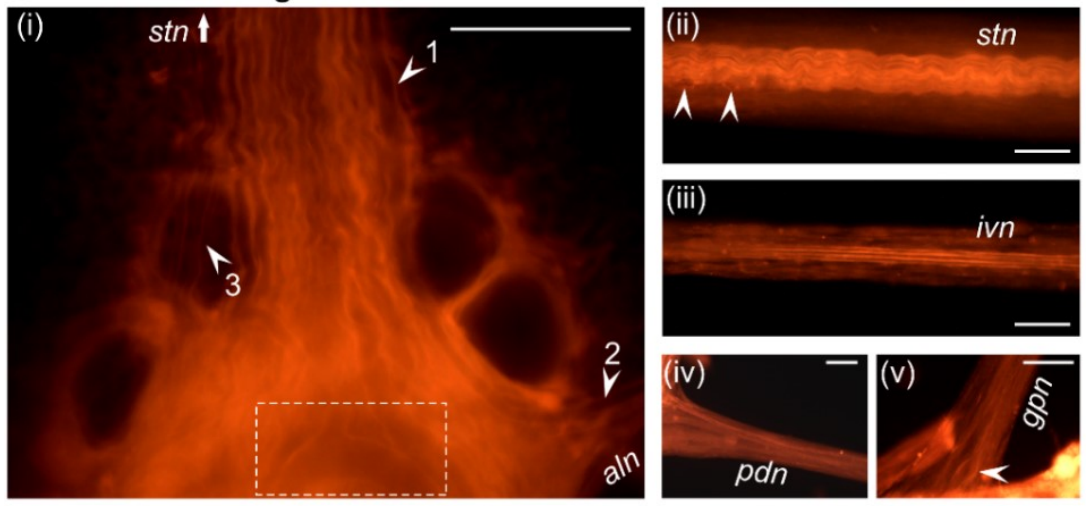

2

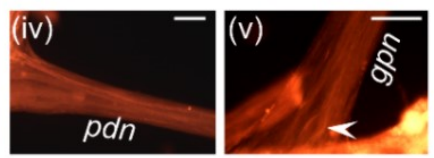

D AGR staining

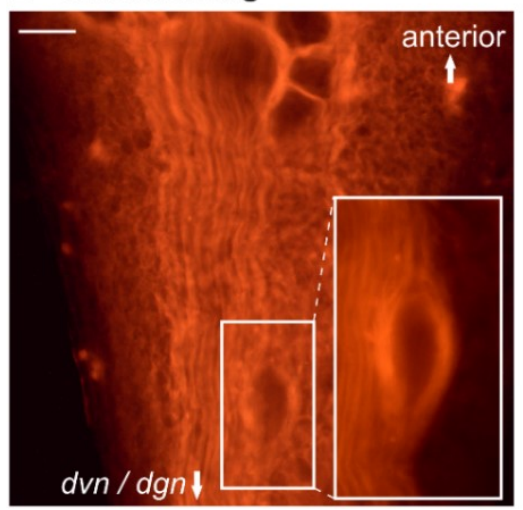

Fig. 3. RH795 staining in the stomatogastric nervous system of the crab, Cancer borealis. A: Cell visibility before (i) and after desheathing (ii) the STG (note that the ganglion was not stained here). Pictures show the same ganglion, illuminated with a 
dark-field condenser and taken using the same light intensity, magnification, and camera settings. $n$, neuropil; m, motor neurons; * minuten pin. Scale bar is $100 \mu \mathrm{m}$. B: RH795 stains STG neurons through the ganglion sheath and causes a clear and longlasting staining of neural structures. Comparison of visibility of the non-desheathed ganglion during $180 \mathrm{~min}$ of washout of the dye (left 5 pictures) and after desheathing (right). Immediately after removing the dye, the sheath still contained a lot of dye (1 min), but contrast and visibility improved quickly. The dye caused a long-lasting staining through the ganglion sheath without excessive residual staining of the sheath. Visibility did not further improve after desheathing the ganglion. Scale bar is $50 \mu \mathrm{m}$. C: RH795 revealed fine neuronal structures like neurites, axons and varicosities. (i) Anterior part of the STG showing individual axons entering the ganglion via the stn (see arrows). Distinct neurites in the STG neuropil could be identified, which is impossible with regular light microscopy. (ii) Middle part of the non-desheathed stn. Individual axons and varicosities (arrows) were visible after staining. (iii) Staining of the ivn, (iv) the pdn and (v) the gpn. Arrow indicates the location of the soma of the gastro-pyloric receptor neuron GPR. Scale bar is $100 \mu \mathrm{m}$. D: The AGR soma shown here stained with RH795 is anatomically isolated from other STG neurons, located more posteriorly than the somata of STG motor neurons. Scale bar is $50 \mu \mathrm{m}$. 
A AGR ff. oscillations disappear after desheathing

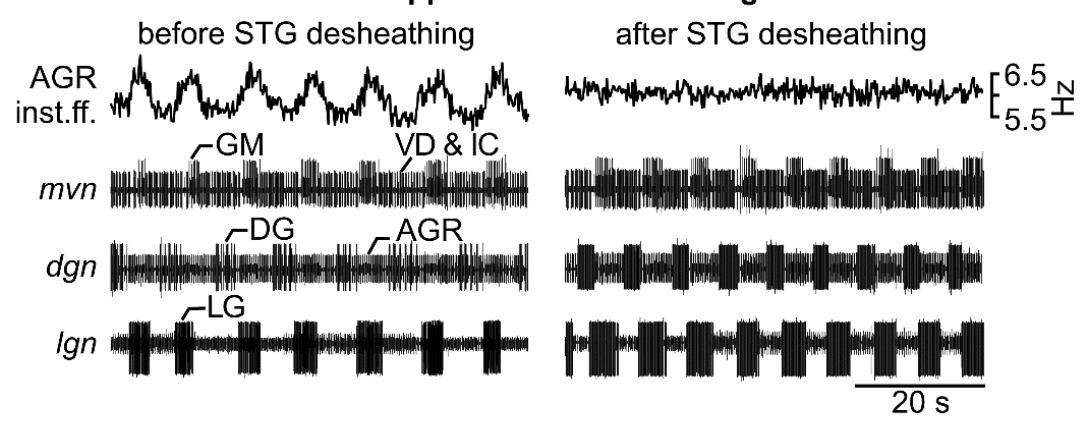

B Phase diagram of AGR inst. ff. and the gastric mill motor neurons
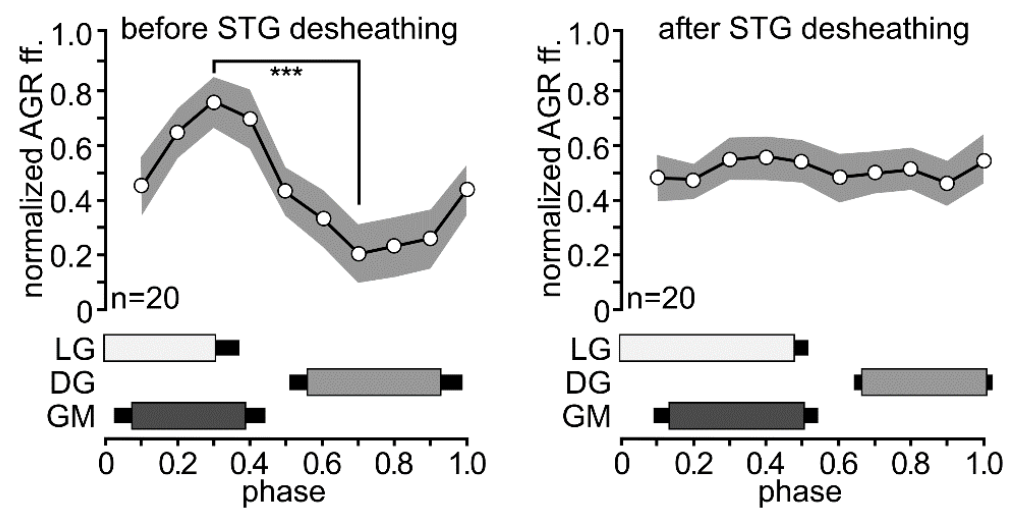

C AGR inst. ff. oscillations are not affected by RH795

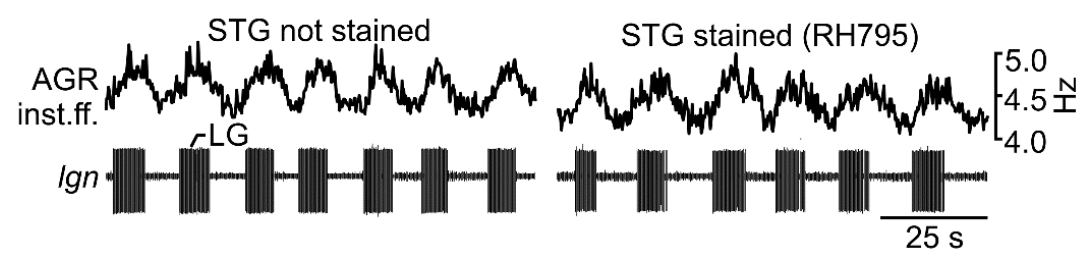

D Phase diagram of AGR inst. ff. and the gastric mill motor neurons
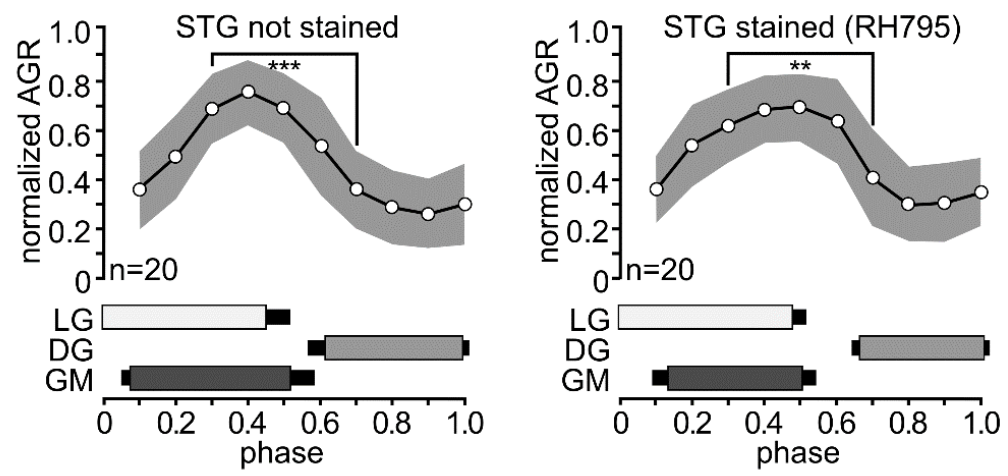

Fig. 4. Desheathing causes subtle changes in neuronal activity. A: Rhythmic changes in AGR inst. ff. disappear after desheathing. During a gastric mill rhythm, AGR inst. ff. (top trace) changed rhythmically. Bottom traces: extracellular recordings of $m v n$, dgn and Ign showing the timing of the gastric mill rhythm. Left: before desheathing; right: after 
desheathing the STG. Raw data are included in supplemental S3. B: Phase diagram of normalized averaged AGR inst. ff. and the gastric mill motor neurons LG, GM and DG before (left) and after (right) desheathing, from the same animal. AGR inst. ff. was binned (bin width 0.1). The grey surface shows the standard deviation. ${ }^{* * *}$ significant difference with $p<0.001$. C: Rhythmic changes in AGR inst. ff. were not affected by RH795. Left: non-desheathed STG, before staining, right: non-desheathed STG after 1 $\mathrm{h}$ staining and 20 minutes washout. D: Phase diagram of the same animal before (left) and after (right) staining with RH795. For detail see (B). Phases 0.3 and 0.7 were significantly different. ${ }^{* * *} p<0.001$ before staining and ${ }^{* *} p<0.01$ after staining. There was no significant difference between the mean values of all phases before and after staining (see text for details). 
A
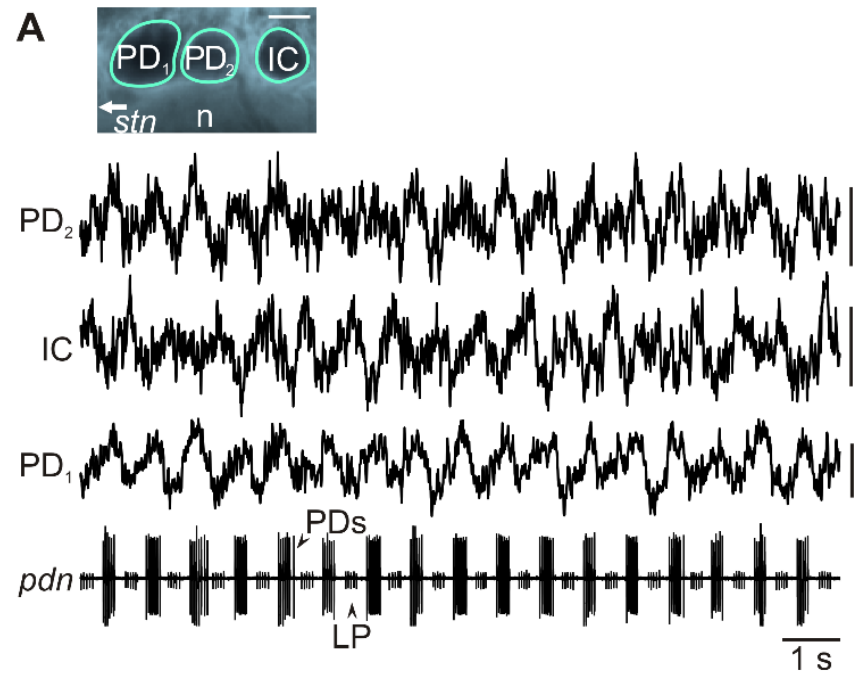

B Cycle-triggered average

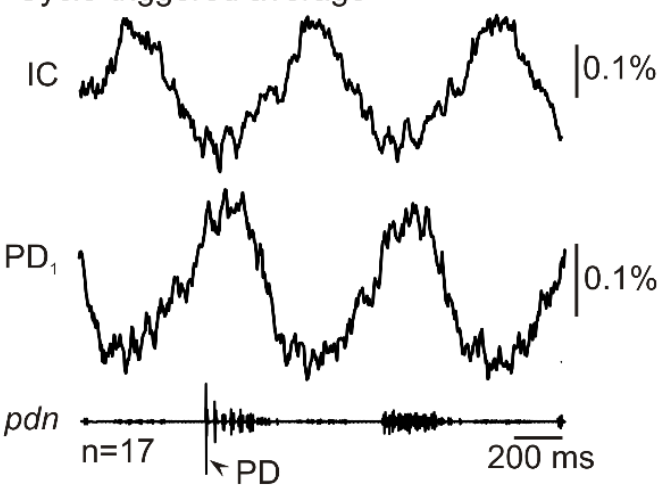

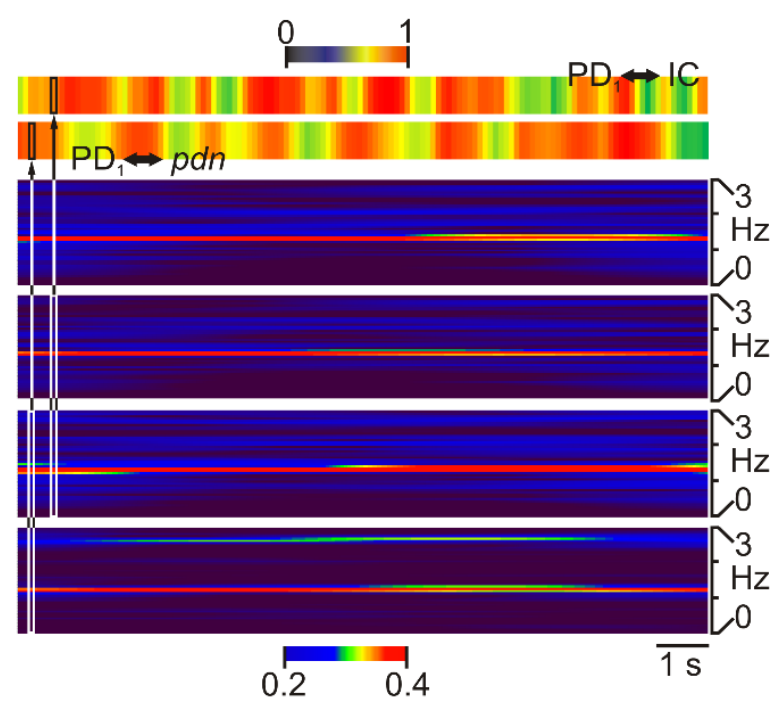

C Spike-triggered average

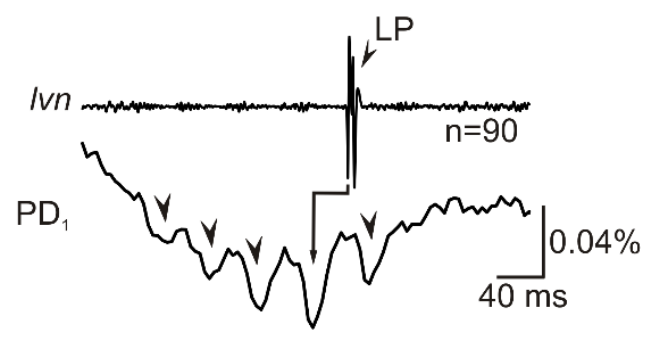

Fig. 5. Optical imaging through the ganglion sheath. A: Left, Single-sweep simultaneous optical recording of IC and the two PD neurons, along with an extracellular recording of the pdn. In this particular recording the pdn also shows the action potentials of LP. Top: Photo showing the three somata selected for recording. Scale bar is $50 \mu \mathrm{m} ; \mathrm{n}$, neuropil. PDs and IC showed rhythmic and alternating changes in their fluorescence. Vertical scale bars are $0.04 \%$. Right: spectrogram showing the major frequency components of optical and extracellular recordings. Warmer colors indicate higher power. Top traces, correlation of frequency distribution $(1-10 \mathrm{~Hz})$ for each time point for PD and pdn, as well as for PD and IC. There was a high correlation of the frequency components at all points in time. Note the different time scale (slightly compressed to fit page). B: Cycletriggered average of PD and IC, plus pdn, showing the phase dependence of the optical signal. The first PD spike in each cycle was used for triggering. C: LP spike-triggered average of Ivn and PD optical recording showing LP-timed synaptic inhibition in PD and its temporal dynamics. 

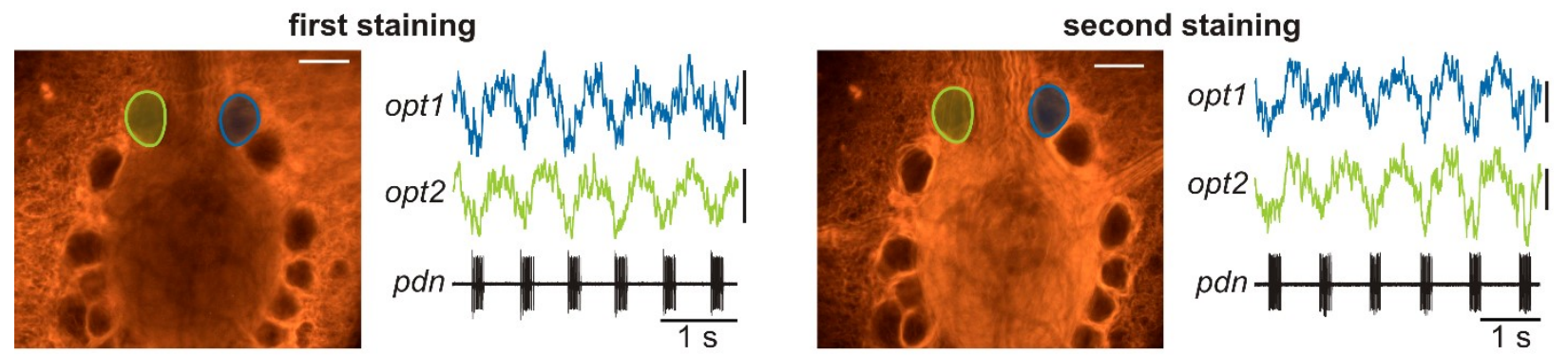

Fig. 6. Repetitive staining permits long-term experiments. Photos with regions of interest and simultaneous single-sweep recording of two pyloric neurons, plus pdn. Left: first staining; right: second staining. Recordings were taken roughly 5 hours apart from one another. Staining duration was 1 hour in each case. Scale bars: $100 \mu \mathrm{m}$ (photo) and $0.1 \%$ (optical recordings). 

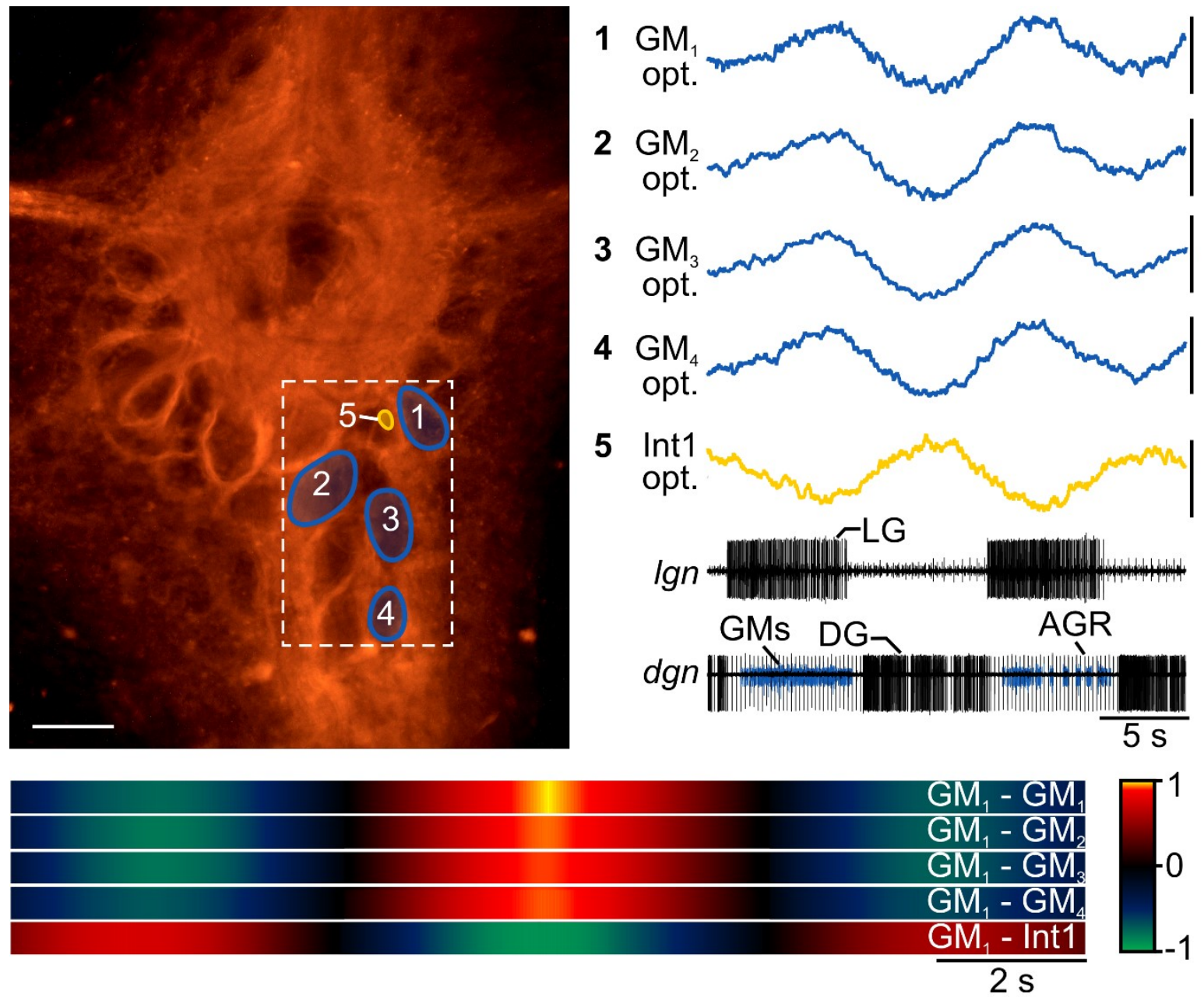

Fig. 7. RH795 allows the simultaneous recording of gastric mill neurons through the ganglion sheath. Left: photo of STG with 5 regions of interest representing the locations of the $4 \mathrm{GM}$ neurons and Int1. Actual optical recording region indicated by dotted-white lines. Scale bar is $100 \mu \mathrm{m}$. Right: simultaneous optical recording of GMs and Int1. Scale bars are $0.4 \%$. Bottom traces: extracellular recordings of Ign and dgn, showing the burst activities of LG, DG and GMs. Note that GM spikes (blue) are small, preventing their individual identification on the extracellular recording. Bottom: Waveform correlation of GMs and Int1, showing high synchrony of the $4 \mathrm{GMs}$ and antiphasic correlation of GMs and Int1. Colors code correlation factor (1: strong correlation; 0 : no correlation; -1 : strong antiphasic correlation). $\mathrm{GM}_{1}$ was used as a reference for all correlations, with the top trace showing the $\mathrm{GM}_{1}$ autocorrelation. 
CHAPTER II: SPATIAL DISTRIBUTION OF INTERMINGLING POOLS OF PROJECTION NEURONS WITH DISTINCT TARGETS: A 3-DIMENSIONAL ANALYSIS

\title{
OF THE COMMISSURAL GANGLIA IN CANCER BOREALIS
}

\begin{abstract}
Projection neurons play a key role in carrying long-distance information between spatially distant areas of the nervous system and in controlling motor circuits. Little is known about how projection neurons with distinct anatomical targets are organized, and few studies have addressed their spatial organization at the level of individual cells. In the paired commissural ganglia (CoGs) of the stomatogastric nervous system of the crab Cancer borealis, projection neurons convey sensory, motor, and modulatory information to several distinct anatomical regions. While the functions of descending projection neurons (dPNs) which control downstream motor circuits in the stomatogastric ganglion are well-characterized, their anatomical distribution as well as that of neurons projecting to the labrum, brain, and thoracic ganglion have received less attention. Using cell membrane staining, we investigated the spatial distribution of CoG projection neurons in relation to all CoG neurons. Retrograde tracing revealed that somata associated with different axonal projection pathways were not completely spatially segregated, but had distinct preferences within the ganglion. Identified dPNs had diameters larger than $70 \%$ of CoG somata and were restricted to the most medial and anterior $25 \%$ of the ganglion. They were contained within a cluster of motor neurons projecting through the same nerve to innervate the labrum, indicating that soma position was independent of function and target area. Rather, our findings suggest that CoG neurons projecting to a variety of locations follow a generalized rule:
\end{abstract}


for all nerve pathway origins, the soma cluster centroids in closest proximity are those whose axons project down that pathway.

\section{Introduction}

Neuronal circuits are composed of many neurons organized in spatial arrangement that support computation and flow of information in the nervous system. Analyses of neuropilar projection patterns and soma locations in a number of systems have demonstrated that sensory and motor structures in the brain are highly organized, and preserved across individuals of a given species. In some invertebrates this applies even to the level of individual cells, providing detailed maps of the locations of identified neurons or classes of neurons and how they are clustered (Borst, 2014; Brandt et al., 2005; Burrows, 1996; Busch et al., 2009; Hsu and Bhandawat, 2016; Jefferis et al., 2007; Wolff et al., 2015). The spatial architecture of nervous system regions other than sensory and motor structures is much less studied, potentially because complex brain functions may depend on less organized nervous system structures. Determining the anatomy of regions that integrate sensory information and control motor functions, including the number of involved neurons as well as their sizes and locations, is an essential step toward the characterization and identification of the involved neurons. Descending projection neurons (dPNs) are involved in the control and selection of motor circuits. Their actions are best studied in rhythmic motor systems such as central pattern generator (CPG) circuits. CPGs govern many behaviors and the most vital functions of the body such as walking, breathing and chewing, as well as locomotion and saccadic eye movements (Briggman and Kristan, 2008; Marder and Bucher, 2001; Selverston, 2010; Stein, 2009). In both vertebrates and invertebrates, CPGs are under 
continuous control by descending inputs that, in addition to their direct synaptic actions, may release a plethora of different neuromodulators in a paracrine fashion. This allows CPGs to generate a variety of behaviors ranging from categorically different to variants of a single motor act within the same behavioral category (Barlow and Estep, 2006;

Briggman and Kristan, 2008; Dickinson, 2006; Kiehn, 2006; Stein, 2009). Although there has been an effort to understand the structures underlying descending control and their function in motor pattern output in various systems (Goulding, 2009; Grillner, 2003; Lemon, 2008; Orger et al., 2008), a detailed description of the anatomical organization of distinct classes of dPNs is lacking.

The CPGs in the stomatogastric nervous system of the crab, Cancer borealis, provide a convenient system to study descending control of motor circuits. The pyloric (filtering) and gastric mill (chewing) CPGs in the stomatogastric ganglion (STG) are controlled by dPNs located in the paired commissural ganglia (CoGs) (Stein, 2009). The CoGs act as hubs for integrating information from the supra-esophageal ganglion ('brain') and the thoracic ganglion (Kirby and Nusbaum, 2007). CoG neurons are involved in controlling several motor activities, including cardiac, esophageal, gastric mill and pyloric rhythms (Meyrand and Marder, 1991; Meyrand et al., 1991; Nusbaum and Beenhakker, 2002). They also process sensory information from multiple modalities (Blitz et al., 2004; Blitz et al., 2008; Diehl et al., 2013; Hedrich et al., 2011; Hedrich et al., 2009). Several dPNs that innervate the STG have been identified (Blitz et al., 1999; Coleman and Nusbaum, 1994; Norris et al., 1994; 1996), with distinct activities, cotransmitters and effects on the STG motor patterns (Nusbaum and Beenhakker, 2002; Stein, 2009), allowing us to study a functionally diverse set of neurons with single-cell 
resolution. However, little information is available regarding the anatomical organization of the CoGs with respect to the positions of neurons and their overall distribution in the ganglion.

The CoGs are bilaterally symmetrical ganglia, hypothesized to be derived from the tritocerebrum or mandibular neuromere (Vilpoux et al., 2006). It has been estimated in other species to contain around 390 neurons (Wiersma, 1957). The dPNs reach the unilateral STG via the stomatogastric nerve (stn; fig. 1), by projecting through one of two pathways, either the inferior (ion) or the superior esophageal nerve (son). We used these features to selectively stain dPNs and identify their soma locations in the CoG performing a combination of retrograde axonal tracing, whole-ganglion fluorescent imaging and 3D reconstruction. Additionally, among the few hundred neurons in the ganglion, we show the location of ascending neurons to the brain and descending neurons to the thoracic ganglion, facilitating future investigation on the functions of CoG neurons. Moreover, when considered with these populations of ascending neurons and other ganglionic landmarks, our analyses of soma location and size provide a guide for the spatial distribution of dPNs.

\section{Methods}

\section{Dissection}

Adult crabs (Cancer borealis) were delivered from The Fresh Lobster Company (Boston, MA) or Ocean Resources Inc. (Sedgwick, ME). Crabs were kept in tanks with artificial sea water made from artificial sea salt (salt content $\sim 1.025 \mathrm{~g} / \mathrm{cm}^{3}$; Instant Ocean Sea Salt Mix, Blacksburg, VA). Tanks were kept at a temperature of $11^{\circ} \mathrm{C}$ and a 12-hour light-dark cycle. Animals were anesthetized on ice for 20 minutes before 
dissection. We used isolated nervous systems to perform all of our experiments. As described previously (Blitz and Nusbaum, 1997), the nervous system was pinned down in a silicone elastomer-lined (ELASTOSIL RT-601, Wacker, Munich, Germany) Petri dish and continuously superfused $(7-12 \mathrm{ml} / \mathrm{min})$ with $C$. borealis saline $\left(10-13^{\circ} \mathrm{C}\right)$. Physiological crab saline consisted of: $\mathrm{NaCl}, 440 \mathrm{mM} ; \mathrm{KCl}, 11 \mathrm{mM} ; \mathrm{MgCl}_{2}{ }^{*} 6 \mathrm{H}_{2} \mathrm{O}, 26$ $\mathrm{mM}$; $\mathrm{CaCl}_{2}, 13 \mathrm{mM}$; trisma base, $10 \mathrm{mM}$; maleic acid, $5 \mathrm{mM}(\mathrm{pH} 7.4-7.6)$.

\section{Staining of neuronal membranes}

The voltage-sensitive dye Di-4-ANEPPDHQ was used to indiscriminately stain neural membranes. Di-4-ANEPPDHQ is a lipophilic fluorescent dye that preferably stains neuronal membranes in the stomatogastric nervous system (Goldsmith et al., 2014) and highlights cell somata. A 5 mM stock solution was prepared by diluting $1 \mathrm{mg}$ dye in 300 $\mu \mathrm{l}$ of DMSO, aliquotting for single-staining use, and kept in darkness at $-20^{\circ} \mathrm{C}$. Immediately before bath-application, the stock solution was diluted 1:1 with pluronic acid F-127 (20\% solution; Biotium, Hayward, CA) DMSO solution, and mixed with saline to the final concentration of $50 \mu \mathrm{M}$. A petroleum jelly well was built around the desheathed CoG and 50-100 $\mu \mathrm{l}$ of Di-4-ANEPPDHQ were bath-applied to the well. The dye was applied for 30-60 minutes, after which the petroleum jelly well was removed and the whole preparation was bathed in cooled $\left(10-13^{\circ} \mathrm{C}\right)$ saline for the remainder of the experiment.

\section{Retrograde labeling}

A petroleum jelly well was constructed around the nerve that contained the axons to be retrogradely labeled (fig. 1B). The saline was removed from the well and first rinsed 3-5 times with ultrapure water. The nerve was then transected, the well was filled with $\mathrm{NiCl}_{2}$ 
or $\mathrm{CoCl}_{2}$ (5\%-8\%; Sigma, St. Louis, MO), or a mix of the two, and the preparation was incubated at $4{ }^{\circ} \mathrm{C}$ for $1-5$ days. For long incubation periods ( $>2$ days) we supplemented the saline with Penicillin-Streptomycin (Sigma) at $10 \mathrm{mg} / \mathrm{L}$ to prevent bacterial contamination. After incubation, the $\mathrm{NiCl}_{2}$ was removed from the well and replaced with saline. The well was then removed, and the entire Petri dish was rinsed ( $3-5$ times) with normal saline. 25-30 drops of saturated dithiooximide (Sigma) solution were placed in the dish ( $\sim 1$ drop for every $\mathrm{ml}$ of saline) to precipitate the Nickel. After a 20-30 min application, the dish was washed several times with normal saline. Following this treatment, the $\mathrm{NiCl}_{2}$-filled somata appeared dark blue. The CoG was then desheathed and counterstained with Di-4-ANEPPDHQ to visualize all other CoG neurons. Nonneuronal dye affinity was low enough to visibly exclude any sheath staining from subsequent analyses (Goldsmith et al., 2014; Preuss and Stein, 2013). Optical imaging and picture processing For describing the 3D structure of the CoG, we used a 5 mega pixel color CMOS camera (TCA-5.0C, Ample Scientific LLC, Norcross, GA, USA) and TSView software (Version 7.3.1.7, Tucsen Imaging Technology Co., Fujian, China). Fluorescent excitation light was provided by a narrowband LED with $470 \mathrm{~nm}$ (CoolLED, Yorktown Heights, NY) and fluorescence emission was detected using a quadband filter cube (Semrock, Inc, Rochester, NY). Excitation light intensities and imaging exposure time varied and were adjusted to the individual preparation. We used a $10 \times$ objective (UMPlanFL N, NA 0.30, WD 3.3 mm, cc=water; Olympus Corporation, Tokyo, Japan) mounted on an upright epifluorescence microscope (modified BX51, Scientifica, East Sussex, UK). The photos were taken at a spatial resolution of $1994 \times 2592$ pixels every 2 
$\mu \mathrm{m}$ in the dorsoventral axis. The stack of photos for each preparation was imported to and manipulated in Amira 3D analysis software (version 6.0.0, FEI, Visualization Sciences Group, http://www.fei.com/software/amira-3d-for-life-sciences/, RRID:SCR_014305). Directionally, soma 3D locations were quantified along the anteroposterior (A-P), mediolateral (M-L), and dorsoventral (D-V) axes. Soma sizes were measured in the focal plane where the diameter was maximal. The measurements were exported as a data file (csv, coma separated value) for further analysis in Matlab. Figures and data analysis

To compare soma location across preparations, we first calculated the CoG average dimensions (A-P, M-L, and D-V axes). The A-P length of the ganglion was measured as the distance between the anterior border of the neuropil and the most posterior neuron. The M-L length was measured as the distance between the most medial neuron and the most lateral neuron. The $\mathrm{D}-\mathrm{V}$ extent of the ganglion was measured as the distance between the center of the most dorsal and ventral somata. The CoG shape in two dimensions was represented using an ellipse with major and minor axis lengths given by the average A-P and $M-L$ lengths, respectively. This average ganglion is used as reference for comparing ganglia across preparations. The position of each cell in a particular ganglion was mapped onto its corresponding position in the average ganglion using the proportionality between the size of that particular ganglion and the size of the average ganglion.

For the two-dimensional distribution plots we created a grid of bins shaded with respect to density of somata in each bin, placed within the average ganglion represented by the ellipse. Bin size is given by the corresponding scale bars. To 
investigate the overlap between the distinct clusters of neurons we calculated an average contour for each cluster by extracting in each preparation an individual polygon outlining the spread of the stained neurons of that particular cluster. Then an average contour was created by interpolating the average distances from the average center to the vertices of the polygons. For the two CoG neurons projecting axons through the inferior oesophageal nerve (ion) to the stn (Coleman et al., 1995; Norris et al., 1996) (denoted as 'ion dPNs'), we instead used the smallest rectangle that enclosed the two cells, using the $M-L$ and A-P distances as sides of the rectangle.

To describe the relative location of ion dPNs with respect to landmarks in the CoG, we first performed a translation operation centering the ganglion on the landmark in consideration. Either the neuropil or the largest cell (L-cell) was used (Robertson and Moulins, 1981). The Euclidian distances between the landmark and the somata were determined along with the corresponding angle in the A-P and M-L directions. In this representation each vector denotes the position (distance and angle) of the corresponding soma with respect to the landmark.

To characterize the distances from projection pathway origin to the centroid of soma clusters we first determined the projection pathway origin locations in each preparation. The ion and son pathway origin location were determined as the exit point of axon bundle at the ganglion border. Since the entire lateral portion of the CoG borders the commissure, we defined the thoracic ganglion and brain pathway origins as the points where axons were visible at the most anterior and posterior borders of the CoG, respectively. For centroid-to-origin measurements, distances were determined from the centroids to each individual (non-averaged) projection pathway origin. 
For statistical analysis, Excel (version 2010-2013 for Windows, Microsoft), MATLAB, version R2014b (http://www.mathworks.com/products/matlab/, RRID:SCR_001622), or SigmaPlot version 11.0 (Systat Software, San Jose, CA) were used. Data are given as mean \pm SD unless otherwise noted. "N" denotes the number of CoGs used. In all figures significance is indicated using * $(p<0.05)$. Statistical tests utilized were paired t-test, Pearson correlation coefficient, Mann-Whitney U rank-sum test, and Kruskal-Wallis one-way ANOVA on ranks using the Student-Newman-Keuls test for multiple comparisons. Final figures were prepared with MATLAB and CorelDraw, version X7 for Windows (http://www.coreldraw.com, RRID:SCR_014235).

\section{Results}

\section{General structure of the commissural ganglion}

Gaining insight into the anatomical features of the CoG neuronal population was at the core of our investigation. Wiersma (1957) estimated the number of CoG cells in the crayfish, Procambarus clarkii, at about 390 , and follow up studies used this estimate to give an approximate number of cells in other crustacean species. C. borealis is a prime system for studying motor pattern modulation and selection, as it both controls downstream motor circuits and integrates information from several regions of the nervous system (Nusbaum and Beenhakker, 2002; Stein, 2009). However, neither the number nor the overall organization of CoG neurons have been determined.

To establish an anatomical description of the CoG and its neurons, we first characterized ganglion size, number of cells, soma diameters and locations. In this system, endogenous markers with neuronal specificity have yet to be established. Thus, we exogenously applied a lipophilic fluorescent dye (Di-4-ANEPPDHQ) to the CoGs and 
acquired images at several focal depths ( $2 \mu \mathrm{m}$ steps, see Materials and Methods), resulting in a stack of images (fig. 2A). Soma boundaries were determined by tracing the cell membrane in three dimensions. Since each soma covered several focal planes, the size and location of somata were measured in the focal plane with the largest cell diameter. Regions without detectable somata and very dense staining were defined as neuropil.

In many invertebrate ganglia, the somata cover or are arranged around a neuropil- a region of densely clustered neurites (Richter et al., 2010). Fig. 2B shows a three-dimensional (3D) reconstruction of the somata of one CoG. Darker shading represents somata located more dorsally and lighter shading more ventrally located ones. In all ganglia stained ( $\mathrm{N}=51$ ganglia), there was a clear distinction between neuropil areas and areas with somata. The neuropil region was typically located in the most anteromedial section of the ganglion (marked with an "x" in fig. 2C). In contrast, the posterior parts of the ganglion were comprised mostly of neuronal somata. To determine the anteroposterior (A-P) length of the ganglion we thus used the anterior border of the neuropil and the most posterior neuron. The mediolateral (M-L) length was measured as the distance between the most medial neuron and the most lateral neuron. The dorsoventral (D-V) extent of the ganglion was measured as the distance between the center of the most dorsal and ventral somata. The center of each neuron was estimated as the mid-point of the soma in the $D-V$ plane where its maximal diameter occurred. On average, the ganglion dimensions (in $\mu \mathrm{m}$ ) were $487.77 \pm 49.09$, $606.03 \pm 54.5$, and $105.84 \pm 27.76$ for the $M-L, A-P$, and $D-V$ axes, respectively $(N=51)$. The total number of CoG somata ranged from 89-220 somata per ganglion (average: 
$151.63 \pm 32.39$ somata, $N=51$; total of 7,729 somata) and was thus lower than in the estimates of all previous studies. Neither soma number nor soma size was correlated to ganglion size ( $p>0.9$ for both).

To characterize the spatial distribution of CoG somata, we first approximated the CoG shape in two dimensions using an ellipse with major and minor axis lengths given by the average A-P and M-L lengths, respectively. We then mapped the somata of all preparations onto the average ellipse using a proportionality relationship (see Material and Methods). Next, we created a 2D density plot displaying the density of somata at different locations in the ganglion, with lighter regions corresponding to lower ( $\sim 5$ somata per bin) and darker regions corresponding to higher ( $\sim 50$ somata per bin) soma densities (fig. 3A; $N=51 ; 7729$ somata). The anteromedial portion of the ganglion was always devoid of somata and was exclusively comprised of neuropil. Somata were distributed throughout the remainder of the ganglion, with the highest density in the middle of the ganglion and a slight shift in the posterolateral direction. Soma density decreased towards the borders of the ganglion.

Soma diameter within each ganglion covered a wide-range $(2.5-136.97 \mu \mathrm{m})$ as indicated by the histogram shown in fig. 3B $(16.93 \pm 11.68 \mu \mathrm{m} ; \mathrm{N}=51)$, with the highest occurrence at $12 \mu \mathrm{m}$, corresponding to $23 \%$ of all somata ( $\mathrm{N}=51 ; 1808$ of 7729 somata). There was an obvious skew towards smaller diameters, and this feature was consistent across preparations. Somata with diameters smaller than $20 \mu \mathrm{m}$ represented $76.4 \%$ (5911 of 7729 somata) of the distribution, with an average diameter of $12.24 \pm 3.79 \mu \mathrm{m}$ $(\mathrm{N}=51)$. The remaining somata included medium size somata $(20-50 \mu \mathrm{m} ; 21.2 \%$, with an average diameter of $27.98 \pm 7.01 \mu \mathrm{m}$ ) and $2.3 \%$ with somata diameters $>50 \mu \mathrm{m}$ 
(70.42 $\pm 7.01 \mu \mathrm{m} ; 175$ of 7729 somata; fig. 3B, inset). To determine a relationship between soma size and location in the CoG, we plotted the spatial distribution of the soma diameter along the A-P, M-L, and D-V axes (figs. 3C-E). Most small-diameter cells were distributed approximately equally on the A-P axis, with the highest density between 200 and $600 \mu \mathrm{m}$ (fig. 3C). In the M-L direction they were found between 300 and $500 \mu \mathrm{m}$ (fig. 3D). In contrast, larger cells were located rather centrally, with posterior and medial shifts (fig. 3C,D). Yet, in general, there was a higher density of CoG somata towards the anterior and lateral portions of the ganglion. There was no obvious D-V preference according to size; most somata were located in the dorsal half of the ganglion (fig. 3E). Smaller somata ( $<20$ micron diameter) were mostly found centrally in the A-P, M-L and D-V axes, with a tendency to more dorsal locations. No statistically significant relationship was found between soma diameter and location throughout the CoG.

Descending projection neuron distribution in the commissural ganglion The CoG hosts various types of neurons, including local interneurons, stomatogastric motor neurons and long-distance PNs (Meyrand et al., 1994). Among the latter there are dPNs that innervate the CPG circuits in the STG and play an important role in motor pattern selection and plasticity. While the transmitter content and physiological actions of several dPNs have been identified, their spatial organization in the CoG is not well known.

To study the soma location of these dPNs in the CoG, we retrogradely filled their axonal projections from the STG with $\mathrm{NiCl}_{2}$ (see Materials and Methods) and counterstained the ganglion with Di-4-ANEPPDHQ to visualize all other CoG neurons. 
Coleman et al. (1992) identified up to 20 neurons in each CoG that project axons to the STG. These axons project either through the ion or the son before they enter the unilateral stn (see fig. 1). In many sensory and motor systems, neurons are organized somatotopically, with somata organized in distinct clusters depending on their projection pathways (Schieber, 2001). Alternatively, there is evidence that somatotopy may also result from functional congruency, independent of projection pathway (Zullo and Hochner, 2011). To address this question, we studied dPNs spatial organization separately for each pathway. First, we selectively stained dPNs projecting to the STG via the son by backfilling the stn and transecting the ipsilateral ion to prevent labeling of non-son dPNs (inset in fig. 4A). We obtained an average of $4.3 \pm 1.3$ somata per ganglion (ranging from 3 to 8 son dPNs labeled, $\mathrm{N}=12$ ). Soma diameters ranged from 16 to $45 \mu \mathrm{m}$ (fig. $4 \mathrm{~A})$, with an average of $33.6 \pm 6.9 \mu \mathrm{m}(\mathrm{N}=12)$. The son $\mathrm{dPNs}$ were located primarily in the medioposterior quadrant of the CoG (fig. 4B). Along D-V direction, on average, son dPNs were located $37.7 \pm 20.2 \mu \mathrm{m}$ from the dorsal surface of the ganglion (fig. 4C). No son dPNs were found ventral of $90.1 \mu \mathrm{m}$.

To identify the locations of the dPNs that innervate the STG via the ion, we backfilled the stn axons after transection of the son (fig. 4D, inset). Modulatory commissural neurons 1 and 5 (MCN1 and MCN5) are the sole dPNs projecting via the ion (Coleman et al., 1995; Norris et al., 1996). Our staining thus selectively marked MCN1 and MCN5. Accordingly, we consistently stained two somata (8 of 10 stainings; a single cell in the two remaining stainings) per ganglion. Soma diameters ranged from 29.94 to $45.67 \mu \mathrm{m}$ and were on average $37.15 \pm 4.34 \mu \mathrm{m}$ (fig. $4 \mathrm{D}, \mathrm{N}=10$ ). They were distinct from most other somata in the ganglion as their diameters differed by more than 
a standard deviation from the mean of all somata $(16.93 \pm 11.68 \mu \mathrm{m}$, see also fig. $3 \mathrm{~B})$. Similar to the son dPNs, somata were preferentially located in the medioposterior quadrant of the ganglion (fig. 4E). On average their D-V location was found $33.89 \pm 11.47$ $\mu \mathrm{m}$ from the dorsal surface of the ganglion. Additionally, given that there are only two ion dPNs, we designated the most posterior ion $\mathrm{dPN}$ as 'ion $\mathrm{dPN} 1$ ' and the other as 'ion dPN2'. We found a consistent difference in the D-V locations of the two ion dPNs: the ion dPN2 was located more dorsally than the ion dPN1 ( $p<0.05, N=8$; paired t-test; fig. 4C). No significant differences in location were found when comparing the locations of the ion dPNs with those of the son dPNs (Mann-Whitney $U=43.0, n$ (ion dPNs) $=8$, $n($ son $\mathrm{dPNs})=12, P=0.728)$.

Across all preparations, there appeared to be overlap between the locations of the son and of the ion dPNs (fig. 4B, C,E), suggesting a cell organization that is independent of projection pathway. To examine the extent of this overlap we obtained contour lines for each preparation, outlining the clusters of son and ion dPNs, respectively (see Materials and Methods). The average contours for all preparations along with the standard deviations indicate that the ion dPNs cluster is mostly contained within the son dPNs cluster (fig. 4F), showing that indeed there is overlap in soma location of dPNs that innervate STG motor circuits via distinct pathways.

\section{Organization of projection neuron clusters}

The spatial distribution of the ion dPNs and son dPNs showed that ion dPNs are contained within the son dPNs cluster, suggesting that soma location is dependent on projection target rather than on projection pathway. If this were true, other neurons projecting through the same pathways but with distinct targets should have spatially 
distinct clusters. The ion bifurcates after leaving the CoG: the continuation of the ion which connects to the stn and the labral nerve, which projects anteriorly towards the labrum. While the latter most likely contains the axons of motor neurons innervating the labral muscles, the first contains the dPNs MCN1 and MCN5 that innervate the STG. We used this feature to test whether the somata of projection neurons that innervate the STG have distinct locations from those of neurons projecting through the labral nerve, i.e. whether or not assemblages of neurons sharing similar projection pathways but with distinct targets overlap in the localization of their somata.

For this, we transected the ion between the CoG and labral nerve and backfilled all ion axons, including STG projection neurons and labral motor neurons. Soma diameters ranged from 10 to $57 \mu \mathrm{m}$ (fig. $5 \mathrm{~A}$ ), with an average of $31.1 \pm 9.7 \mu \mathrm{m}(\mathrm{N}=9)$. We obtained an average of $11.8 \pm 4.5$ somata per ganglion (ranging from 7 to $21, \mathrm{~N}=9$ ). Somata were located mostly in the medial portions of the CoG, with a slight preference for the posterior area (fig. 5B). Furthermore, no distinct clusters for ion dPNs and other labral nerve neurons were obvious, suggesting overlap of ion dPNs and labral nerve motor neurons. In fact, when we compared the averaged contours of ion $\mathrm{dPNs}$ and ion dPNs plus labral nerve there was almost complete overlap in their 2D location (fig. 5C). The spatial distribution of the ion dPN plus labral nerve somata along the D-V axis (fig. $5 \mathrm{D})$ shows that on average somata were found $45.0 \pm 15.1 \mu \mathrm{m}(\mathrm{N}=9)$ from the dorsal surface of the ganglion. Other than the fact that ion dPN somata were located slightly more dorsally than most of the labral nerve somata, there was no obvious segregation into distinct clusters. 
Our results did not reveal any spatial segregation between neurons according to pathways or targets. To test whether this was generally true for CoG projection neurons, we expanded upon the work of Kirby and Nusbaum (Kirby and Nusbaum, 2007) and mapped soma locations of neurons projecting to the brain and the thoracic ganglion (TG) with respect to the son, ion, and labral nerve soma distributions already described.

To label somata projecting to the TG we retrogradely filled axons with $\mathrm{CoCl}_{2}$ in the large commissure between CoG and TG. On average, $27 \pm 8.8$ somata $(\mathrm{N}=4 ; 17-38$ somata) were labeled. As previously reported (Kirby and Nusbaum, 2007), most somata were found lateral and distributed equally along the A-P axis (fig. 6B). The coefficients of variation for the A-P (0.11) and M-L (0.3) directions were low, indicative of little variability between animals. Soma diameters ranged from 11.1 to 95.5 microns $(31.3 \pm 15.2 \mu \mathrm{m}, \mathrm{N}=4)$. This included a large number of medium sized somata $(20-50 \mu \mathrm{m}$, $62 \%$ of the distribution), with 3 to 7 characteristically larger somata $(50-100 \mu \mathrm{m}, 25 \%$ of the distribution; fig. 6A). A more thorough examination of the relationship between soma diameter with location demonstrated that larger somata were located more posteriorly in relation to smaller somata (fig. $6 \mathrm{C}$ ). However, there was no obvious preference of location according to size when considering the $M-L$ and $D-V$ axes (figs. 6D,E).

Using the same approach, we labeled CoG neurons projecting to the brain. Backfills stained on average $45.7 \pm 14.3$ somata per ganglion (range 14-66 somata; $\mathrm{N}=11$ ). Most of the brain-projecting neurons were of small to medium size ranging from 8 to $50 \mu \mathrm{m}$ comprising $98 \%$ of the somata $(17.3 \pm 5.5 \mu \mathrm{m}, \mathrm{N}=11$, fig. $6 \mathrm{~F})$. Consistently one large cell with average diameter of $96.1 \pm 11.5 \mu \mathrm{m}(\mathrm{N}=11)$ was located in the posteromedial portion of the ganglion. This cell had previously been identified (L-cell; 
(Robertson and Moulins, 1981)). Its average location is depicted with a triangle in fig. 6G. The somata of brain-projecting neurons were consistently located in the posterior portion of the ganglion with no preference for M-L or D-V axes (fig. $6 \mathrm{H}-\mathrm{J}$ ). Their location was consistent across animals with small variability $(C V=0.28$ and $C V=0.19$ for $A-P$ and M-L, respectively). In combination, the contour plots for all clusters (fig. 7) show that somata of projections to the TG were homogeneously distributed throughout the lateral portion of the CoG. Neurons projecting to the brain were consistently clustered in the more posterior regions of the ganglion, overlapping with the posterior range of the TG cluster. However, there was less overlap with son dPNs and even less with ion dPNs clusters. Additionally, the centers of mass ('centroids') of the clusters (markers in fig. 7) seemed conspicuously positioned nearby their projection pathway origins (i.e. where their axonal bundles are first distinguished from the ganglion itself). We tested this by quantifying the relationship of soma cluster centroid location with their projection pathway origin. The angular distribution of projection pathway origins in relation to the center of the averaged ganglion revealed a consistency in origins across preparations (fig. $8 \mathrm{~A}$; bin size 5 degrees; $\mathrm{N}=10$ ). When comparing projection pathway origins (averages shown as six-sided stars, fig. 8B) with the averaged soma cluster centroids (fig. 7,8B), we found that the most proximal cluster to a given pathway was the cluster whose axons were associated with a given pathway (fig. 8C-F). Spatial preferences of $d P N s$ In some arthropods, soma locations are well preserved across individual animals for a given species (Burrows, 1996). Our data showed the locations of different soma clusters in the ganglion, but they also indicated considerable variability between animals. For 
example, staining of the two ion dPNs (MCN1 and MCN5) showed the area where these neurons can be found, but it appears that this area varies in location and size in different animals (fig. 4F). The soma locations in the downstream STG motor circuits vary substantially (Bucher et al., 2007), as do the neuropilar projections of the descending PNs in the STG. To test whether the soma locations of the CoG neurons innervating the STG also show variability, we quantified the soma spread in different CoG clusters along the A-P and M-L directions. We calculated the coefficient of variation (CV) using the contour analysis done earlier (fig. 7). While the outer dimensions of the ganglion were rather constant $(C V=0.09$ for $A-P, 0.1$ for $M-L, 0.26$ for D-V directions), we found considerably larger CV's for the son dPNs (CV=0.48 for A-P, and 0.63 for M-L directions). Similarly, there was substantial variability of the ion $\mathrm{dPNs}$ cluster ( $C V=0.63$ and $C V=0.87$ in the A-P and $M-L$ directions, respectively). The collective cluster of the ion $\mathrm{dPNs}$ along and labral motor neurons displayed less variability (A-P: $0.25, M-L: 0.16)$ than the ion $\mathrm{dPN}$ cluster by itself. Thus, the location of MCN1 and MCN5 was more variable than that of the cluster they belong to, indicating that these two dPNs do not have a fixed position within that cluster.

Our analysis so far was in reference to the boundaries of the ganglion, and did not include the possibility of spatial rules within the ganglion itself, such as relative locations, i.e. distance to and orientation towards other cells. Therefore, we selected conspicuous features of the ganglion as landmarks and determined the relative positions of the ion dPNs to the landmarks. We chose the center of the neuropil and the center of the largest cell of the ganglion, the L-cell (Robertson and Moulins, 1981), as landmarks and studied the relative distances of the two ion dPNs (MCN1 and MCN 5) 
with respect to these references (fig. 9A). We first calculated the distance and angle of both ion $\mathrm{dPNs}$ with respect to the neuropil (fig. 9B; $\mathrm{N}=8$ with $16 \mathrm{PN}$ somata). The vector distance was on average $294.3 \pm 52.1 \mu \mathrm{m}$, and the angle of the two ion dPNs spanned only a $36.8^{\circ}$ range (from $231.9^{\circ}$ to $268.7^{\circ}$; average angle of $248.4 \pm 12.6$ degree). This acute angular span measured across several preparations suggests that the neuropil and ion dPNs share at least a directional spatial relationship. Using the L-cell as reference, the average vector distance was $114.9 \pm 41.9 \mu \mathrm{m}$, with a wider span of the ion dPNs angular distribution $244.8^{\circ}$ (ranging from $1.7^{\circ}$ to $246.5^{\circ}$; average angle 127.5 \pm 68.9 degrees, fig. $9 \mathrm{C}$ ). Thus, the variability of the angular positions of the ion dPNs with respect to the neuropil was considerably less than that with respect to the Lcell, i.e. while the ion $\mathrm{dPNs}$ can be located almost anywhere around the L-cell, they are confined into a much smaller angular range when the neuropil is used as a landmark. Additionally, the variability of the distances using the neuropil as landmark was less than the variability obtained when using the $L$-cell as landmark $(C V=0.18$ and $C V=0.36$, respectively).

To test whether distances between dPNs scale with their distances to the landmarks we measured the distances between the ion $\mathrm{dPNs}$ and the two landmarks in each preparation. We found several distances that scaled with one another: the distance from neuropil to the L-cell, for example, scaled with the distance of ion dPN1 to the L-cell. There was a significant negative correlation between neuropil - ion dPN1 and L-cell - ion dPN1 distances $(R=-0.75, p=0.03$; fig. $9 D, N=8)$, i.e. in preparations where the ion dPN1 - neuropil distance was large, the ion dPN1 - L-cell distance was small. Taking the ion dPN1 as reference, we found a positive correlation between ion dPN1- 
neuropil and ion $\mathrm{dPN} 1-\mathrm{dPN} 2$ distances, although not significant $(\mathrm{R}=0.671$ and $\mathrm{p}=0.069$ (fig. 9E). Similar results were obtained when using the neuropil as reference and its distances to ion $\mathrm{dPN} 1$ and to ion $\mathrm{dPN} 2(\mathrm{R}=0.672$ and $\mathrm{p}=0.068$; fig. 9F). All other combinations of pairs of distances showed no significant correlation, and very low Rvalues.

In summary, if one ion dPN is found far away from the neuropil, the other ion $\mathrm{dPN}$ is far away from the neuropil too, and at a greater distance from the other dPN, meaning that the distances scale. In contrast, if the L-cell is far away from the neuropil, it can be found closer to ion dPN1.

\section{Discussion}

The commissural ganglia: Centers for controlling divergent neuronal outputs Strategically positioned between the brain and the thoracic and stomatogastric motor circuits, the CoGs of $C$. borealis and other decapod crustaceans play an important role in integrating sensory input, processing information from ascending and descending pathways, and sending commands to downstream motor centers (Blitz et al., 2004; Nusbaum and Beenhakker, 2002; Stein, 2009). Similar to other motor control centers (Jefferis et al., 2007; Tanaka et al., 2004; Travagli et al., 2006), the CoGs integrate sensory information from various modalities, including mechano- and proprioceptive sensory structures (Beenhakker and Nusbaum, 2004; Smarandache and Stein, 2007), and descending command signals from the brain (Hedrich and Stein, 2008). The general anatomical features of how motor control centers are organized with respect to the localization of distinct neuronal classes is often unknown. This is in contrast to many sensory neuropils and motor centers, which have rather consistent anatomical features 
across individuals of the same species or even between related species (Brandt et al., 2005; Burrows, 1996; Hsu and Bhandawat, 2016). To address this issue, we used retrograde tracing experiments in combination with whole-ganglion imaging to provide a three-dimensional perspective of soma location in the CoGs. In contrast to the numerically larger motor control centers in vertebrates, CoG neurons and their functions can be identified individually. For example, several command-like CoG neurons (the dPNs) have distinct and identifiable modulatory actions on the downstream motor centers in the STG (Blitz et al., 1999; Coleman and Nusbaum, 1994; Norris et al., 1994; 1996), while other CoG neurons affect the cardiac output (Robertson and Moulins, 1981) or may provide information to thoracic motor centers about the status of CoG and STG activity (Kirby and Nusbaum, 2007).

The general morphology of the CoG was similar across animals and resembled the canonical structure of other invertebrate ganglia with a large neuropil area surrounded by somata. However, our results indicate a considerably smaller number of CoG somata than previously reported, with a maximum of 220 somata in a single CoG. Wiersma (1957) reported 390 neurons in each CoG of the red swamp crayfish, Procambarus clarkii. However, this number was obtained by estimating the total number of neurons from single slides rather than by detailed counts in the ganglion. Since then a number of publications have suggested upwards of 500 neurons per CoG in $C$. borealis (Kirby and Nusbaum, 2007; Marder et al., 1987), but none of them have provided a systematic cell count. Our approach may yield a conservative measurements of CoG soma number, because the dorsal to ventral resolution of the fluorescent staining was 2 microns. The measured number of somata may thus underestimate the 
real number since somata with diameters less than 2 microns may have not been detected. This did not affect the measurements of dPN location and number, as these neurons possess comparatively larger soma diameters and were stained separately by retrograde labeling. Moreover, the distribution of soma diameters (fig. 3B) suggests that somata with diameters less than 2 microns comprise less than $3 \%$ of the total population. Finally, it is well known that soma number varies between species. Even in the relatively well conserved STG, the number of neurons differs between species (Bucher et al., 2007). For example, the STG of $C$. borealis has 26 neurons and that of Homarus americanus has 30 , despite the fact that their activities are almost identical (Stein et al., 2016).

The number of backfilled dPNs we obtained was lower than in a previous study (Coleman et al., 1992). This apparent difference is unlikely to have resulted from technical problems during the retrograde labeling, since it was specific to staining of the son dPNs. In contrast, the same staining protocol consistently revealed the same number of ion dPNs as previously reported. Moreover, when we traced backfilled axons in the opposite direction and counted retrogradely labeled STG neurons, we consistently obtained the same number (7) as previously reported (Coleman et al., 1992). Differently from previous studies, which used Biocytin, we carried out our experiments using $\mathrm{NiCl}_{2}$. Biocytin has been implicated to cross gap-junctions (Marx et al., 2012), with the potential of staining additional neurons indirectly via their coupling to the backfilled neurons. Biocytin staining may thus overestimate the number of somata. $\mathrm{NiCl}_{2}$, on the contrary, does not cross gap junctions, providing a more conservative estimate of the number of descending neurons. 
Clustering and variability of projection neuron location

Sensory inputs and motor outputs are often represented as organized maps in the nervous system. This fundamental organization of processing has been shown on a macroscopic level in large brain regions such as the vertebrate sensory and motor cortices (Bednar and Wilson, 2015; Graziano, 2016), but also on the level of individual neurons in several invertebrate species (Borst, 2014; Burrows, 1996; Chiang et al., 2011; Hemmi and Tomsic, 2012; Wolff et al., 2015). While sensory or motor maps can be organized as a topographic representation of the body, they are often also organized by function. For example, in the olfactory system of insects and mammals, the somata of secondary sensory neurons are arranged in glomeruli of neurons that process information from olfactory receptor neurons of the same type that are spatially distributed (Grosse-Wilde et al., 2011; Haase et al., 2010; Hallem and Carlson, 2006; Homberg et al., 1988; Lofaldli et al., 2010; Shen et al., 2013; Xu et al., 2003). Likewise, swim interneurons in zebrafish spinal cord are spatially clustered and recruited depending on swim speed (Ausborn et al., 2012). In some invertebrates, there are detailed maps for soma location in all or parts of the ganglia. Notably, leech ganglia have been described in exquisite detail (Briggman and Kristan, 2006; Muller et al., 1981).There are examples demonstrating variability in soma location, as is the case in the crustacean STG (Bucher et al., 2007). This differs strongly from the canonical, very stereotyped activity generated in the STG (Bucher et al., 2005). The motor neuron neurites and the axon terminals of the descending PNs that innervate them have no fixed organization in the STG either (Bucher et al., 2007). The reason for this variability is unclear, but it may be related to the fact that differently from most other aarthropod 
ganglia, the STG is not bilaterally symmetric and may thus not require a strict spatial segregation. Rather, the STG collects information from the bilaterally symmetric CoGs and distributes motor commands equally to the two sides of the stomach.

In contrast to sensory and motor structures, very little is known about how functionally diverse neurons intermingle in motor control centers, and how consistent these anatomical features are when comparing across individuals of the same species. We found that soma locations in the CoGs also varied between individuals, although certain preferences where maintained. There are several potential reasons for the observed variability. Though not much has been elucidated in regards to CoG ontogeny, certain developmental restrictiaons have been shown to impact the neuronal anatomy. For example, sensory input can influence how the nervous system develops, and distinct inputs may differentially target particular groups of neurons (Burrows, 1996). In locusts, new neurons can even be formed in the brain well into late-embryonic development. There is some ambiguity on the ontogenetic origins of the CoGs, but CoG formation may be a result of the fusion of the tritocerebral and mandibular neuromeres (Harzsch, 2002; 2003). It is conceivable that neurons associated with different neuromeres or their precursors are differentially influenced by developmental pressures and extrinsic inputs to the two neuromeres during development, yielding distinct anatomical fusion layouts in individual animals.

There are also functional reasons as to how and why variability in soma position might arise. While not much is known about the activity patterns of most CoG neurons (i.e. neurons projecting to the labrum, brain, and TG), dPN functions are consistent across animals (Stein, 2009). The responses of dPNs to sensory stimulation, as well as 
their effects on the STG motor circuits and axonal projection patterns are well-preserved in all animals. Thus, variability in soma position does not appear to affect the functioning of CoG PNs. CoG neurons, like many invertebrate neurons, are unipolar or pseudounipolar. Their somata may thus not necessarily be active components in signal processing, similar to the variably located STG neurons (Bucher et al., 2007). Most processing in unipolar neurons is achieved at the level of the neurites, with only electrotonic remnants of activity reaching the somata (Burrows, 1996). Along with the passive role in neuronal processing, there is a general lack of synapses on the somata of unipolar and pseudounipolar neurons. This is in contrast with cells having bipolar and multipolar morphologies (e.g. pyramidal neurons in cortex or hippocampus), which require signals to pass through the soma to communicate information from their dendritic fields to their axonal projections. In these neurons, the properties of the somatic compartment and synapses thereon will influence the cells' outputs (Williams and Stuart, 2003). Consequently, soma morphology and location may matter for signal propagation in the latter scenarios. Furthermore, while in sensory structures the projection pathways and soma locations seem to be critical, our data indicate that this may not be the case for centers that process sensory information and control motor circuits. To maintain functional consistency of CoG neurons in different individuals, it is at least understood that neuritic structure and positioning are pivotal when considering proximity to functional partners. Neurites of the dPN MCN1, for example, are consistently compartmentalized with its presynaptic partners, even though soma location varies (Swallie et al., 2015). This indicates that variability in soma location may be the rule and not the exception in these types of neural centers. In consideration of 
this, soma location differences coinciding with maintenance of functional output across animals supports the hypothesis that many solutions may exist to the same problem. This concept has recently been highlighted by the fact the functional output of a circuit is more important than the specific contributing physiological properties, such as synaptic of intrinsic properties of neurons. For example, vastly different combinations of ion channel conductances can lead to the same functional output and the permissible parameter space is evident in the large variability of these conductances between animals of the same species (Drion et al., 2015; Gutierrez et al., 2013). The advent of new genetic and molecular tools (Clark et al., 2008; Garcia et al., 2015; Krenz et al., 2014; Moroz et al., 2008; Schulz et al., 2007; Temporal et al., 2014) will further allow testing this concept by determining genetic cell identities and the cellular distribution of receptors and ion channels. Our study supports that this concept also applies to anatomical structures. Alternatively, variable soma locations may indicate a 'good enough' solution, where the variability of soma location is small enough to not affect functional output.

\section{Preferential locations of projection neurons}

The locations of certain dPNs were independent of function or target area. For example, MCN1 and MCN5 were part of the cluster of neurons that project axons through the ion. This cluster also contains the motor neurons of the labrum and the esophageal motor neuron, i.e. neurons with a different target area and function. MCN1 and MCN5's positions were variable even within this cluster, indicating that while the general area where they can be found is preserved, the precise location of distinct modulatory neurons is not. The strongest preference for soma location was found when comparing 
clusters according to projection pathway. The center of mass of the clusters belonging to different projection pathways were preferentially closer to the origin of their projection pathway than to others. For example, dPNs projecting from the CoG to the STG were restricted to about the most medial $25 \%$ of ganglion. Understanding the organization of neuronal structures is both a major technical challenge and a major undertaking in unraveling the integrative properties of the nervous system. This is particularly true for areas of the nervous system with apparent unstructured or variable organization. Relatively little is known about the functional and anatomical organization of descending neurons in motor control (but see Hsu and Bhandawat (2016)), despite their importance for motor pattern selection and modulation (Berkowitz et al., 2010; Card, 2012;

Deliagina et al., 2008; Dubuc and Grillner, 1989; Herberholz and Marquart, 2012; Kozlov et al., 2014; Le Ray et al., 2011; Nusbaum, 2013; Schuster, 2012; Stein, 2009). This is in part because of a dichotomy between functional and anatomical studies: functional studies are often concerned with one type or a particular group of neurons, while anatomical studies may not or only superficially address neuronal function. This is particularly true for motor control regions in larger and less accessible nervous systems. In contrast, invertebrate systems such as the crustacean stomatogastric nervous system allow the identification of distinct individual motor command neurons and their anatomy. Our results indicate that, at least in the stomatogastric nervous system, somata of individual dPNs are not rigidly fixed in relation to other cells when comparing across different CoGs. We found several general features which unify dPN somata in the CoGs: they are located within a narrow range of soma diameters, and are larger than most non-dPN somata. They also have a preferred location within the ganglion 
and, at least for the two ion dPNs, we found a specific distance from and angle to the neuropil. Used together, these features may provide a guide to locate dPNs. This should be particularly useful for studies which require access to the molecular or physiological properties of one or several dPNs. For example, intracellular recordings with sharp microelectrodes first require the finding and the identification of cells, a laborious, time-consuming and often daunting task in a ganglion with several hundred neurons. Similarly, techniques like optical imaging which allow for simultaneous recording of whole neuronal populations require background knowledge of the distribution of neuronal populations within the ganglion.

Rather than absolute coordinates, landmarks can be helpful in localizing particular pools of neurons. Neuroanatomical atlases represent the structural properties of the nervous system in a common spatial framework. Unlike their geographical counterparts, however, anatomical objects exist in large numbers, one in each animal's nervous system, and no two of them are identical. While the general features of the nervous system in a particular species resemble each other, specific neuronal structures differ in location, size and shape. One way to deal with this issue is to provide a frame of reference based on averaged features from a number of animals (Brandt et al., 2005). Another approach is to instead use landmarks within each ganglion and to create a frame of reference that scales with the landmark distances. Indeed, our results demonstrate that the latter was superior to locate $\mathrm{dPNs}$ in the CoG. For example, we found that the distance between the largest cell (the L-cell) and the neuropil was inversely related to the distance between the neuropil and dPN, making neuropil and Lcell useful landmarks when attempting to localize the dPNs. 
Used with permission from Follmann R, Goldsmith CJ, Stein W. 2017. Spatial distribution of intermingling pools of projection neurons with distinct targets: A 3D analysis of the commissural ganglia in C. borealis. J Comp Neurol. 525:1827-1843. Copyright 2017 Wiley Periodicals, Inc.

Author Contributions: study concept and design: RF, CG, WS; Acquisition of data: RF, CG; Analysis and interpretation of data: RF, CG, WS; Drafting of the article: RF, CG, WS; Critical revision of the article for important intellectual content: RF, CG, WS. Statistical analysis: RF. 


\section{REFERENCES}

Ausborn J, Mahmood R, El Manira A. 2012. Decoding the rules of recruitment of excitatory interneurons in the adult zebrafish locomotor network. Proc Natl Acad Sci U S A 109(52):E3631-3639.

Barlow SM, Estep M. 2006. Central pattern generation and the motor infrastructure for suck, respiration, and speech. J Commun Disord 39(5):366-380.

Bednar JA, Wilson SP. 2015. Cortical Maps. Neuroscientist:1073858415597645.

Beenhakker MP, Nusbaum MP. 2004. Mechanosensory activation of a motor circuit by coactivation of two projection neurons. J Neurosci 24(30):6741-6750.

Berkowitz A, Roberts A, Soffe SR. 2010. Roles for multifunctional and specialized spinal interneurons during motor pattern generation in tadpoles, zebrafish larvae, and turtles. Front Behav Neurosci 4:36.

Blitz DM, Beenhakker MP, Nusbaum MP. 2004. Different sensory systems share projection neurons but elicit distinct motor patterns. J Neurosci 24(50):1138111390.

Blitz DM, Christie AE, Coleman MJ, Norris BJ, Marder E, Nusbaum MP. 1999. Different proctolin neurons elicit distinct motor patterns from a multifunctional neuronal network. J Neurosci 19(13):5449-5463.

Blitz DM, Nusbaum MP. 1997. Motor pattern selection via inhibition of parallel pathways. J Neurosci 17(13):4965-4975.

Blitz DM, White RS, Saideman SR, Cook A, Christie AE, Nadim F, Nusbaum MP. 2008. A newly identified extrinsic input triggers a distinct gastric mill rhythm via activation of modulatory projection neurons. J Exp Biol 211(Pt 6):1000-1011. 
Borst A. 2014. Neural Circuits for Motion Vision in the Fly. Cold Spring Harb Symp Quant Biol 79:131-139.

Brandt R, Rohlfing T, Rybak J, Krofczik S, Maye A, Westerhoff M, Hege HC, Menzel R. 2005. Three-dimensional average-shape atlas of the honeybee brain and its applications. The Journal of Comparative Neurology 492(1):1-19.

Briggman KL, Kristan WB. 2008. Multifunctional pattern-generating circuits. Annu Rev Neurosci 31:271-294.

Briggman KL, Kristan WB, Jr. 2006. Imaging dedicated and multifunctional neural circuits generating distinct behaviors. J Neurosci 26(42):10925-10933.

Bucher D, Johnson CD, Marder E. 2007. Neuronal morphology and neuropil structure in the stomatogastric ganglion of the lobster, Homarus americanus. J Comp Neurol 501(2):185-205.

Bucher D, Prinz AA, Marder E. 2005. Animal-to-animal variability in motor pattern production in adults and during growth. J Neurosci 25(7):1611-1619.

Burrows M. 1996. The Neurobiology of an Insect Brain. New York: Oxford University Press.

Busch S, Selcho M, Ito K, Tanimoto H. 2009. A map of octopaminergic neurons in the Drosophila brain. The Journal of Comparative Neurology 513(6):643-667.

Card GM. 2012. Escape behaviors in insects. Curr Opin Neurobiol 22(2):180-186. 
Chiang AS, Lin CY, Chuang CC, Chang HM, Hsieh CH, Yeh CW, Shih CT, Wu JJ, Wang GT, Chen YC, Wu CC, Chen GY, Ching YT, Lee PC, Lin HH, Hsu HW, Huang YA, Chen JY, Chiang HJ, Lu CF, Ni RF, Yeh CY, Hwang JK. 2011. Threedimensional reconstruction of brain-wide wiring networks in Drosophila at singlecell resolution. Current biology : CB 21(1):1-11.

Clark MC, Khan R, Baro DJ. 2008. Crustacean dopamine receptors: localization and G protein coupling in the stomatogastric ganglion. J Neurochem 104(4):1006-1019.

Coleman MJ, Meyrand P, Nusbaum MP. 1995. A switch between two modes of synaptic transmission mediated by presynaptic inhibition. Nature 378(6556):502-505.

Coleman MJ, Nusbaum MP. 1994. Functional consequences of compartmentalization of synaptic input. J Neurosci 14(11 Pt 1):6544-6552.

Coleman MJ, Nusbaum MP, Cournil I, Claiborne BJ. 1992. Distribution of modulatory inputs to the stomatogastric ganglion of the crab, Cancer borealis. J Comp Neurol 325(4):581-594.

Deliagina TG, Beloozerova IN, Zelenin PV, Orlovsky GN. 2008. Spinal and supraspinal postural networks. Brain Res Rev 57(1):212-221.

Dickinson PS. 2006. Neuromodulation of central pattern generators in invertebrates and vertebrates. Curr Opin Neurobiol 16(6):604-614.

Diehl F, White RS, Stein W, Nusbaum MP. 2013. Motor circuit-specific burst patterns drive different muscle and behavior patterns. J Neurosci 33(29):12013-12029.

Drion G, O'Leary T, Marder E. 2015. Ion channel degeneracy enables robust and tunable neuronal firing rates. PNAS 112(38):E5361-E5370. 
Dubuc R, Grillner S. 1989. The role of spinal cord inputs in modulating the activity of reticulospinal neurons during fictive locomotion in the lamprey. Brain Res 483(1):196-200.

Garcia VJ, Daur N, Temporal S, Schulz DJ, Bucher D. 2015. Neuropeptide receptor transcript expression levels and magnitude of ionic current responses show cell type-specific differences in a small motor circuit. PMID:18495892 35(17):67866800.

Goldsmith CJ, Städele C, Stein W. 2014. Optical imaging of neuronal activity and visualization of fine neural structures in non-desheathed nervous systems. PLoS ONE 9(7):e103459.

Goulding M. 2009. Circuits controlling vertebrate locomotion: moving in a new direction. Nat Rev Neurosci 10(7):507-518.

Graziano MS. 2016. Ethological Action Maps: A Paradigm Shift for the Motor Cortex. Trends in cognitive sciences 20(2):121-132.

Grillner S. 2003. The motor infrastructure: from ion channels to neuronal networks. Nat Rev Neurosci 4(7):573-586.

Grosse-Wilde E, Kuebler LS, Bucks S, Vogel H, Wicher D, Hansson BS. 2011. Antennal transcriptome of Manduca sexta. Proc Natl Acad Sci U S A 108(18):7449-7454.

Gutierrez GJ, O'Leary T, Marder E. 2013. Multiple mechanisms switch an electrically coupled, synaptically inhibited neuron between competing rhythmic oscillators. Neuron 77(5):845-858. 
Haase A, Rigosi E, Trona F, Anfora G, Vallortigara G, Antolini R, Vinegoni C. 2010. Invivo two-photon imaging of the honey bee antennal lobe. Biomed Opt Express 2(1):131-138.

Hallem EA, Carlson JR. 2006. Coding of odors by a receptor repertoire. Cell 125(1):143-160.

Harzsch S. 2002. From stem cell to structure: neurogenesis in the CNS of decapod crustaceans. The crustacean nervous system: Springer. p 417-432.

Harzsch S. 2003. Ontogeny of the ventral nerve cord in malacostracan crustaceans: a common plan for neuronal development in Crustacea, Hexapoda and other Arthropoda? Arthropod structure \& development 32(1):17-37.

Hedrich UB, Diehl F, Stein W. 2011. Gastric and pyloric motor pattern control by a modulatory projection neuron in the intact crab Cancer pagurus. J Neurophysiol 105(4):1671-1680.

Hedrich UB, Smarandache CR, Stein W. 2009. Differential activation of projection neurons by two sensory pathways contributes to motor pattern selection. $\mathrm{J}$ Neurophysiol 102(5):2866-2879.

Hedrich UB, Stein W. 2008. Characterization of a descending pathway: activation and effects on motor patterns in the brachyuran crustacean stomatogastric nervous system. J Exp Biol 211(Pt 16):2624-2637.

Hemmi JM, Tomsic D. 2012. The neuroethology of escape in crabs: from sensory ecology to neurons and back. Curr Opin Neurobiol 22(2):194-200.

Herberholz J, Marquart GD. 2012. Decision Making and Behavioral Choice during Predator Avoidance. Front Neurosci 6:125. 
Homberg U, Montague RA, Hildebrand JG. 1988. Anatomy of antenno-cerebral pathways in the brain of the sphinx moth Manduca sexta. Cell Tissue Res 254(2):255-281.

Hsu CT, Bhandawat V. 2016. Organization of descending neurons in Drosophila melanogaster. Sci Rep 6:20259.

Jefferis GS, Potter CJ, Chan AM, Marin EC, Rohlfing T, Maurer CR, Jr., Luo L. 2007. Comprehensive maps of Drosophila higher olfactory centers: spatially segregated fruit and pheromone representation. Cell 128(6):1187-1203.

Kiehn O. 2006. Locomotor circuits in the mammalian spinal cord. Annu Rev Neurosci 29:279-306.

Kirby MS, Nusbaum MP. 2007. Central nervous system projections to and from the commissural ganglion of the crab Cancer borealis. Cell Tissue Res 328(3):625637.

Kozlov AK, Kardamakis AA, Hellgren Kotaleski J, Grillner S. 2014. Gating of steering signals through phasic modulation of reticulospinal neurons during locomotion. Proc Natl Acad Sci U S A 111(9):3591-3596.

Krenz WD, Parker AR, Rodgers EW, Baro DJ. 2014. Dopaminergic tone persistently regulates voltage-gated ion current densities through the D1R-PKA axis, RNA polymerase II transcription, RNAi, mTORC1, and translation. Frontiers in cellular neuroscience 8:39.

Le Ray D, Juvin L, Ryczko D, Dubuc R. 2011. Chapter 4--supraspinal control of locomotion: the mesencephalic locomotor region. Prog Brain Res 188:51-70. 
Lemon RN. 2008. Descending pathways in motor control. Annu Rev Neurosci 31:195218.

Lofaldli BB, Kvello P, Mustaparta H. 2010. Integration of the antennal lobe glomeruli and three projection neurons in the standard brain atlas of the moth heliothis virescens. Frontiers in systems neuroscience 4:5.

Marder E, Bucher D. 2001. Central pattern generators and the control of rhythmic movements. Curr Biol 11(23):R986-996.

Marder E, Calabrese RL, Nusbaum MP, Trimmer B. 1987. Distribution and partial characterization of FMRFamide-like peptides in the stomatogastric nervous systems of the rock crab, Cancer borealis, and the spiny lobster, Panulirus interruptus. J Comp Neurol 259(1):150-163.

Marx M, Gunter RH, Hucko W, Radnikow G, Feldmeyer D. 2012. Improved biocytin labeling and neuronal 3D reconstruction. Nat Protoc 7(2):394-407.

Meyrand P, Marder E. 1991. Matching neural and muscle oscillators: control by FMRFamide-like peptides. J Neurosci 11(4):1150-1161.

Meyrand P, Simmers J, Moulins M. 1991. Construction of a pattern-generating circuit with neurons of different networks. Nature 351(6321):60-63.

Meyrand P, Simmers J, Moulins M. 1994. Dynamic construction of a neural network from multiple pattern generators in the lobster stomatogastric nervous system. J Neurosci 14(2):630-644. 
Moroz LL, Kohn A, Garcia V, Bentley-Sloan J, Citarella M, Yu F, Farmerie W, Schulz D, Bucher D. 2008. Neuronal transcriptome of the crab Cancer borealis: Brain, stomatogastric ganglion and identified neurons Neuroscience Meeting Planner Washington, DC: Society for Neuroscience, 2008 Online:30.38/D20.

Muller KJ, Nicholls JG, Stent GS. 1981. Neurobiology of the Leech: Cold Spring Harbor Laboratory.

Norris BJ, Coleman MJ, Nusbaum MP. 1994. Recruitment of a projection neuron determines gastric mill motor pattern selection in the stomatogastric nervous system of the crab, Cancer borealis. J Neurophysiol 72(4):1451-1463.

Norris BJ, Coleman MJ, Nusbaum MP. 1996. Pyloric motor pattern modification by a newly identified projection neuron in the crab stomatogastric nervous system. $J$ Neurophysiol 75(1):97-108.

Nusbaum MP. 2013. Modulatory Projection Neurons. In: Binder MD, Hirokawa, N., Windhorst, U., editor. Encyclopedia of Neuroscience Springer. $p$ http://www.springerreference.com/docs/html/chapterdbid/117087.html.

Nusbaum MP, Beenhakker MP. 2002. A small-systems approach to motor pattern generation. Nature 417(6886):343-350.

Orger MB, Kampff AR, Severi KE, Bollmann JH, Engert F. 2008. Control of visually guided behavior by distinct populations of spinal projection neurons. Nat Neurosci 11(3):327-333.

Preuss S, Stein W. 2013. Comparison of two voltage-sensitive dyes and their suitability for long-term imaging of neuronal activity. PLoS ONE 8(10):e75678. . 
Richter S, Loesel R, Purschke G, Schmidt-Rhaesa A, Scholtz G, Stach T, Vogt L, Wanninger A, Brenneis G, Döring C. 2010. Invertebrate neurophylogeny: suggested terms and definitions for a neuroanatomical glossary. Front Zool $7(1): 29$.

Robertson RM, Moulins M. 1981. A corollary discharge of total foregut motor activity is monitored by a single interneurone in the lobster Homarus gammarus. J Physiol (Paris) 77(8):823-827.

Schieber MH. 2001. Constraints on somatotopic organization in the primary motor cortex. J Neurophysiol 86(5):2125-2143.

Schulz DJ, Goaillard JM, Marder EE. 2007. Quantitative expression profiling of identified neurons reveals cell-specific constraints on highly variable levels of gene expression. Proc Natl Acad Sci U S A 104(32):13187-13191.

Schuster S. 2012. Fast-starts in hunting fish: decision-making in small networks of identified neurons. Curr Opin Neurobiol 22(2):279-284.

Selverston Al. 2010. Invertebrate central pattern generator circuits. Philos Trans R Soc Lond B Biol Sci 365(1551):2329-2345.

Shen K, Tootoonian S, Laurent G. 2013. Encoding of mixtures in a simple olfactory system. Neuron 80(5):1246-1262.

Smarandache CR, Stein W. 2007. Sensory-induced modification of two motor patterns in the crab, Cancer pagurus. J Exp Biol 210:2912-2922.

Stein W. 2009. Modulation of stomatogastric rhythms. J Comp Physiol A 195(11):9891009. 
Stein W, Städele C, Smarandache-Wellmann CR. 2016. Evolutionary aspects of motor control and coordination: the central pattern generators in the crustacean stomatogastric and swimmeret systems. In: Schmidt-Rhaesa A, Harzsch S, Purschke G, editors. Structure and evolution of invertebrate nervous systems: Oxford University Press. $p$ in press.

Swallie SE, Monti AM, Blitz DM. 2015. Anatomical Organization of Multiple Modulatory Inputs in a Rhythmic Motor System. PLoS ONE 10(11):e0142956.

Tanaka NK, Awasaki T, Shimada T, Ito K. 2004. Integration of chemosensory pathways in the Drosophila second-order olfactory centers. Curr Biol 14(6):449-457.

Temporal S, Lett KM, Schulz DJ. 2014. Activity-dependent feedback regulates correlated ion channel mRNA levels in single identified motor neurons. Current biology : CB 24(16):1899-1904.

Travagli RA, Hermann GE, Browning KN, Rogers RC. 2006. Brainstem circuits regulating gastric function. Annu Rev Physiol 68:279.

Vilpoux K, Sandeman R, Harzsch S. 2006. Early embryonic development of the central nervous system in the Australian crayfish and the Marbled crayfish (Marmorkrebs). Dev Genes Evol 216(4):209-223.

Wiersma CA. 1957. On the number of nerve cells in a crustacean central nervous system. Acta Physiol Pharmacol Neerl 6:135-142.

Williams SR, Stuart GJ. 2003. Voltage-and site-dependent control of the somatic impact of dendritic IPSPs. The Journal of neuroscience 23(19):7358-7367. 
Wolff T, lyer NA, Rubin GM. 2015. Neuroarchitecture and neuroanatomy of the Drosophila central complex: A GAL4-based dissection of protocerebral bridge neurons and circuits. The Journal of Comparative Neurology 523(7):997-1037.

Xu F, Liu N, Kida I, Rothman DL, Hyder F, Shepherd GM. 2003. Odor maps of aldehydes and esters revealed by functional MRI in the glomerular layer of the mouse olfactory bulb. Proc Natl Acad Sci U S A 100(19):11029-11034. 


\section{FIGURES}
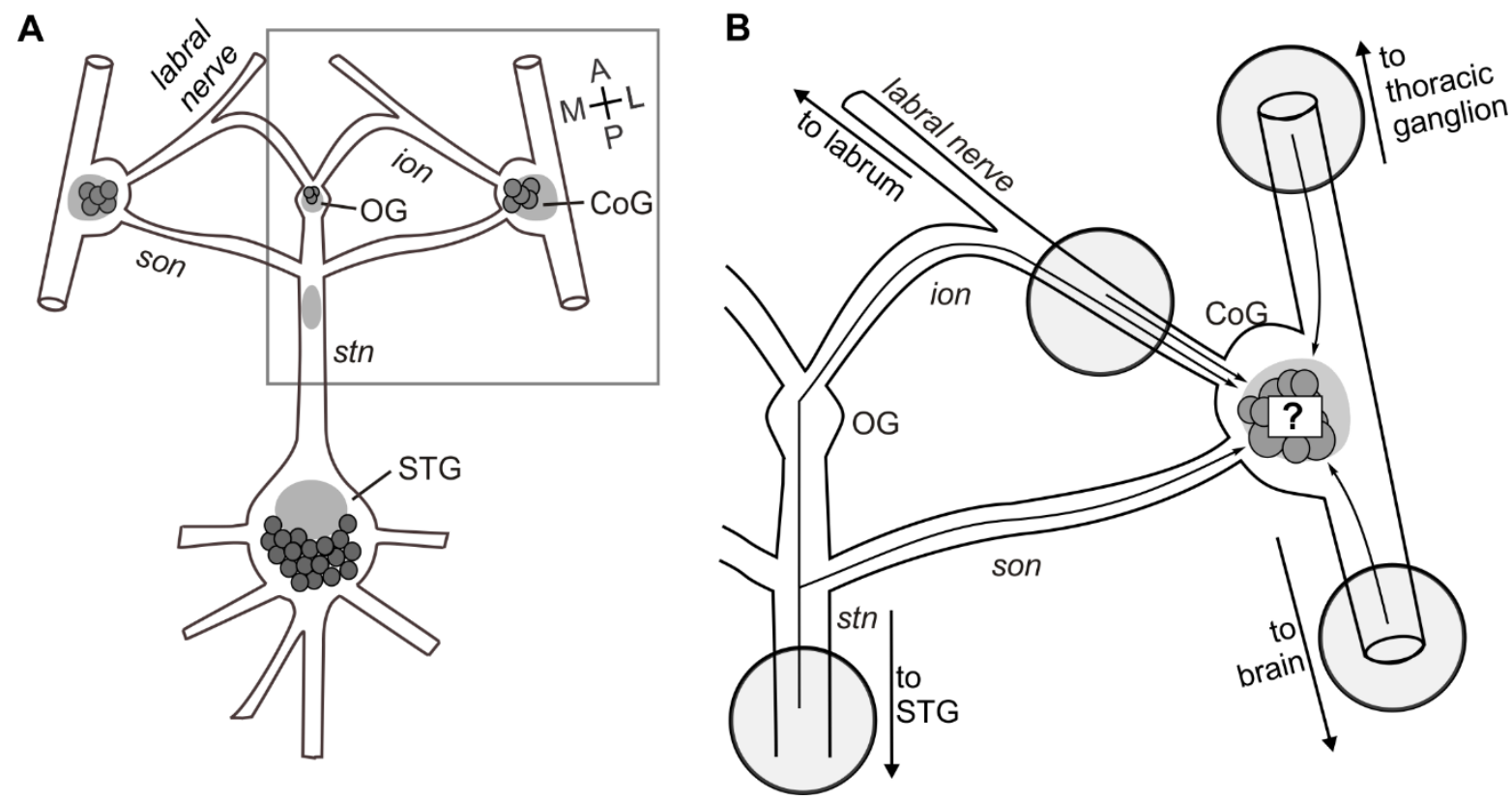

Fig. 1. Schematic representation of the Cancer borealis STNS. A: The STNS is comprised of four ganglia: the OG, the bilaterally paired CoGs, and the STG. B: Magnification of the boxed area in A, showing CoG somata location and arrows indicating their projection pathways. Circles indicate regions where axons were backfilled. STNS = stomatogastric nervous system; OG = esophageal ganglion; CoG = commissural ganglion; STG = stomatogastric ganglion; ion = inferior esophageal nerve; son = superior esophageal nerve; stn = stomatogastric nerve; $\mathrm{A}=$ anterior; $\mathrm{P}=$ posterior; $\mathrm{M}=$ medial; $\mathrm{L}=$ lateral. 

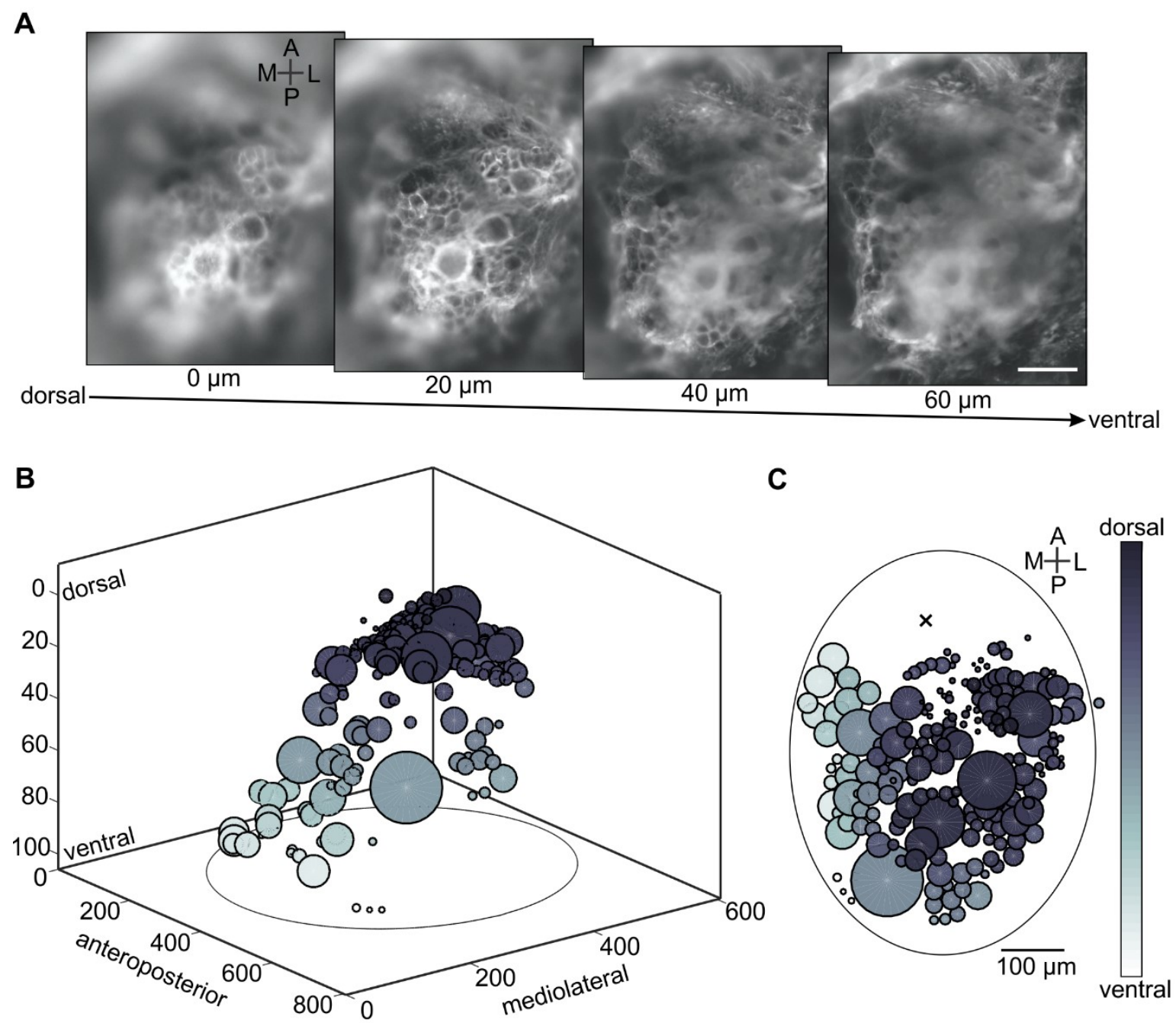

Fig. 2. Description of CoG neurons location in a single preparation. A: Stack of four images of CoG somata with the same anteroposterior (A-P) and mediolateral (M-L) location at different focal depths (depth in the dorsoventral (D-V) direction as indicated). Circular regions with high-contrast staining indicate borders of individual somata. Scale bar, $100 \mu \mathrm{m}$. B: 3D representation of soma locations. Same ganglion as shown in A. Circles represent somata. Circle diameters are proportional to soma diameters and the different shades indicate $\mathrm{D}-\mathrm{V}$ depth from dorsal (darker) to ventral (lighter). C: $2 \mathrm{D}$ projection of the same ganglion on the M-L - A-P plane. Ganglion boundaries (represented by the ellipse) are defined by the outermost regions containing somata or neuropil (centroid marked with ' $X$ '). 
A

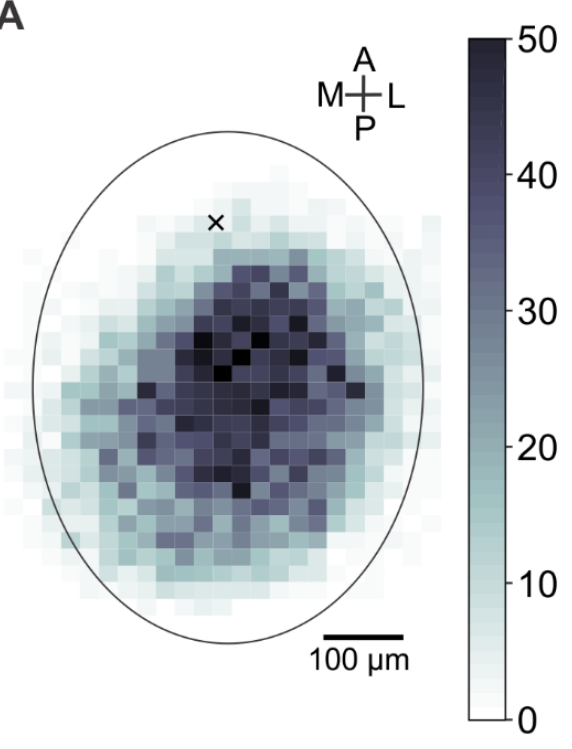

C

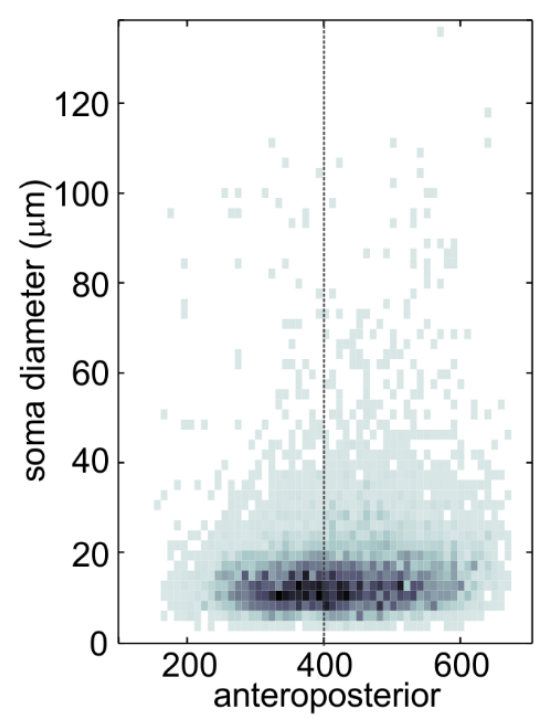

B

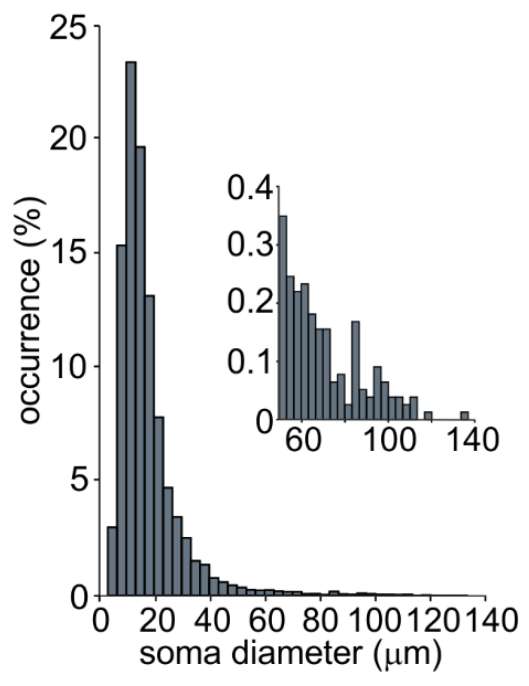

D

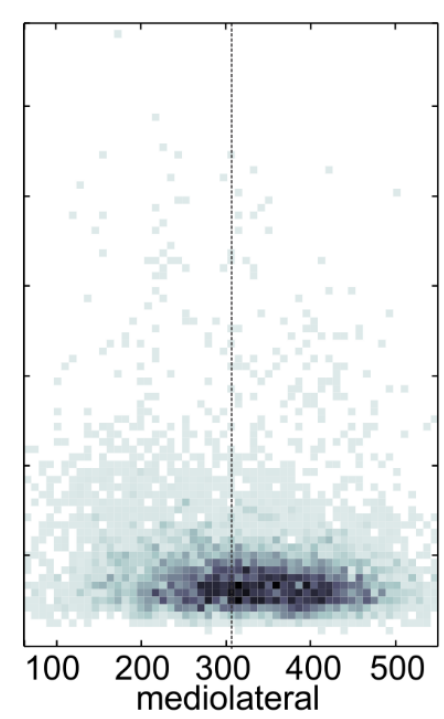

E

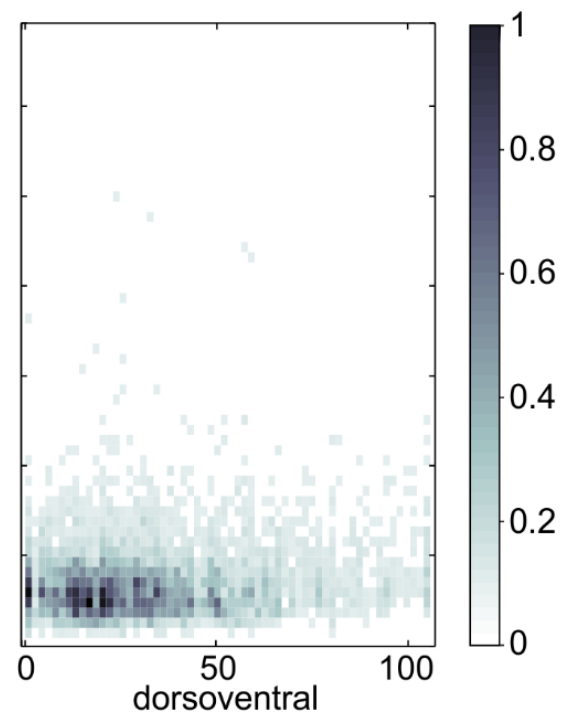

Fig. 3. General description of CoG somata. A: 2D density plot showing the distribution of CoG soma locations for all preparations $(\mathrm{N}=51)$. Lighter and darker shades correspond to lower and higher soma densities, respectively, ranging from 0 to 50 somata per bin (bin size, 20x20 $\mu \mathrm{m}$ ). The neuropil centroid is marked with ' $X$ '. B: Histogram of soma diameters for all ganglia. The inset shows the details for somata with large diameter $(>50 \mu \mathrm{m})$. C-E: Density plots showing the preferred locations of somata with different diameters along the A-P (C), M-L (D), and D-V (E) axes. Dotted lines in C and D indicate the positions of the major and minor axes of the ellipse in A, respectively. Different shades correspond to normalized density of somata for C, D and E. 
A

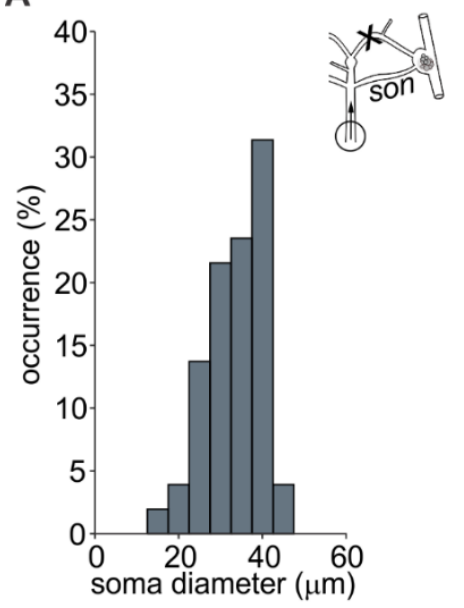

D

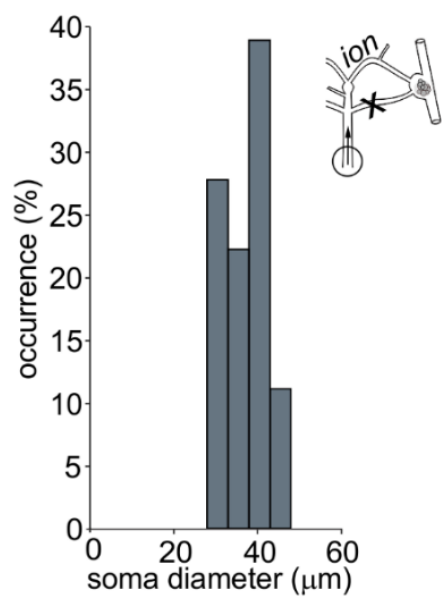

B

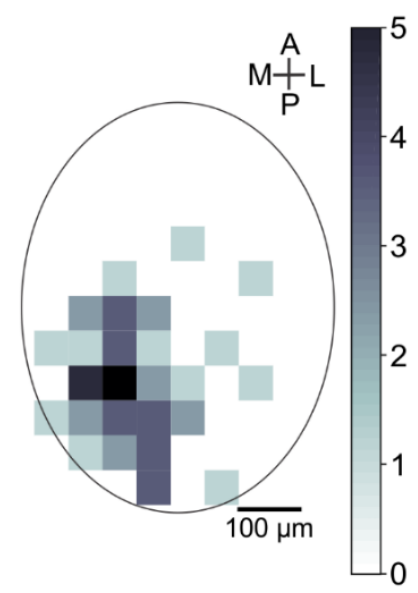

E

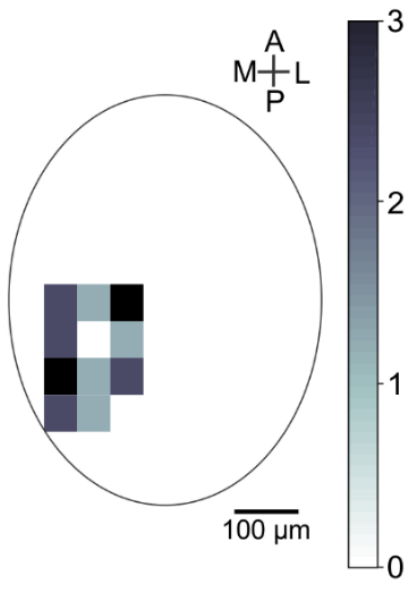

C

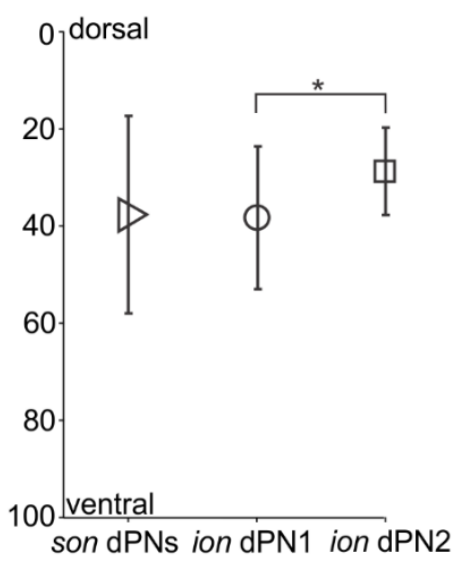

$\mathrm{F}$

Fig. 4. CoG neurons projecting to the STG are mostly posteriorly and medially located. A: Histogram of son PN soma diameter distribution $(N=12)$, inset: schematic of the backfilling of the stn (shaded circle) and transecting the ion $(\mathrm{X})$ to prevent labeling of non-son PNs. B: 2D density plot showing the distribution of son PNs location in the ganglion represented by the ellipse. Number of somata in each bin is as indicated by

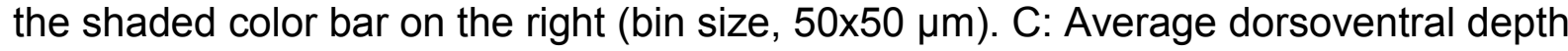
positions of the son $\mathrm{dPNs}$, ion $\mathrm{dPN} 1$, and ion $\mathrm{dPN} 2$ as indicated by the triangle, circle and square symbols, respectively, with the corresponding standard deviation. D: ion $\mathrm{dPN}$ soma diameter distribution $(\mathrm{N}=10)$. Inset: schematic showing where the stn was backfilled (circle). The son was transected (X) to prevent labeling of non-ion PNs. E: 2D density plot showing the distribution of ion dPNs locations. The number of somata in each bin is as indicated by the shaded bar on the right (bin size, $50 \times 50 \mu \mathrm{m}$ ). F: Average contours of son (light grey) and ion (darker rectangle) with corresponding standard deviations (dotted and dashed, respectively). The ion dPNs cluster is almost completely contained within the son dPN cluster in the 2D projection. Additionally, they overlap along the $\mathrm{D}-\mathrm{V}$ direction $(\mathrm{C})$. The asterisk highlights a consistent difference in the $\mathrm{D}-\mathrm{V}$ locations of the two ion dPNs $(p<0.05, N=8)$. 
A

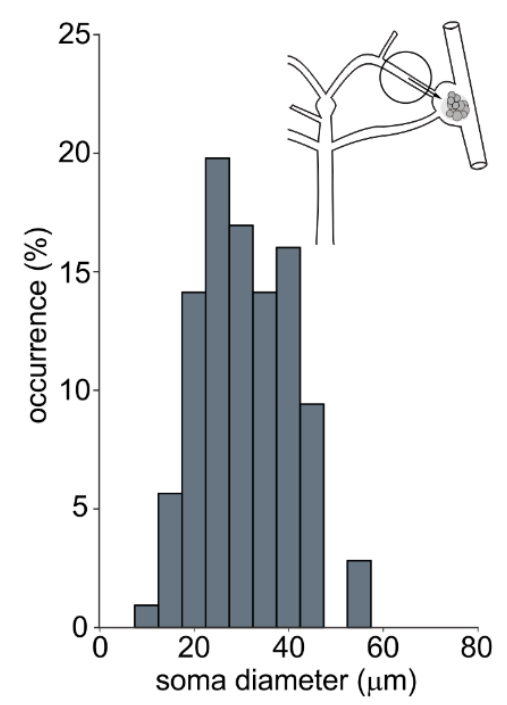

C

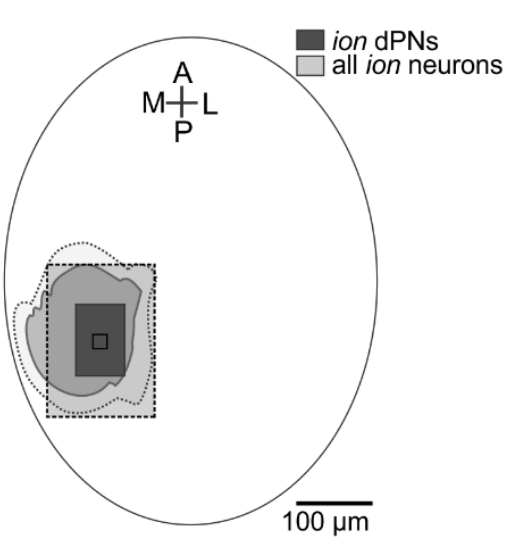

B

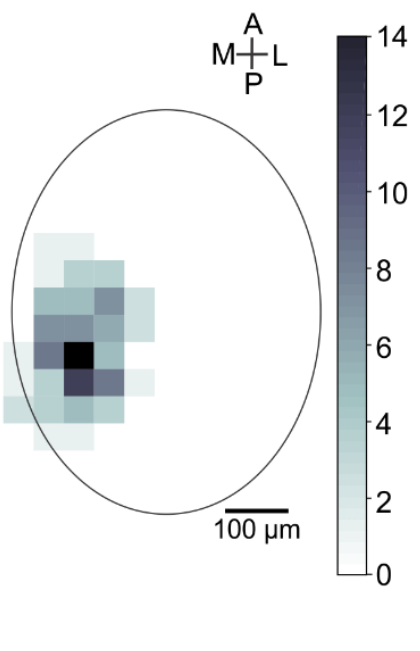

D

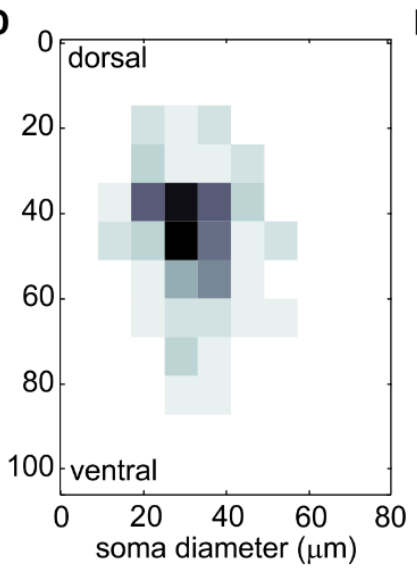

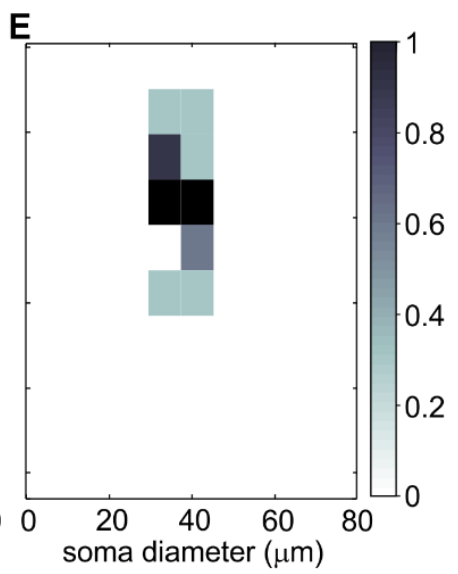

Fig. 5. Neurons with distinct functions and targets overlap in soma localization. A: Histogram of soma diameters for backfilled CoG neurons $(\mathrm{N}=9)$ projecting via the ion, including the two dPNs projecting to the STG (MCN1 and MCN5) and the neurons projecting through the labral nerve. Inset: backfill location (circle). B: 2D density plot showing ion projecting CoG neurons mostly located in medial portions of the ganglion, with a slight preference for the posterior direction. Number of somata in each bin is as indicated by the shaded bar (bin size, 50x50 $\mu \mathrm{m}, \mathrm{N}=9$ ). C: The averaged 2D contours (solid lines) of all CoG neurons projecting axons through the ion (light grey) and only dPNs projecting to the STG (dark grey) overlap, plus standard deviation. D,E: 2D density plots of soma diameter and D-V location show that all CoG neurons projecting via the ion included more ventral locations than dPNs projecting via the ion to the STG alone $(D, E ;$ bins size $40 \times 40 \mu \mathrm{m})$. Different shades of the bar on the right correspond to normalized density of somata for $D$ and $E$. 
A

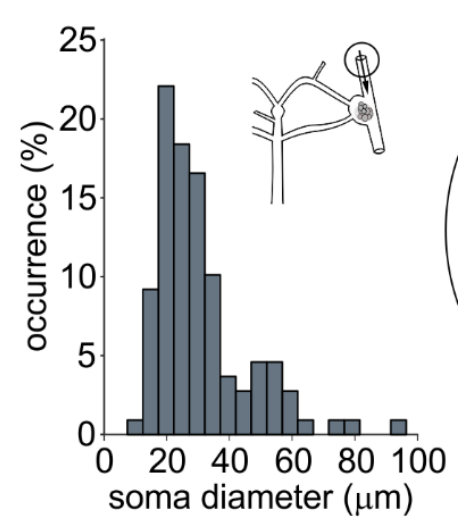

B
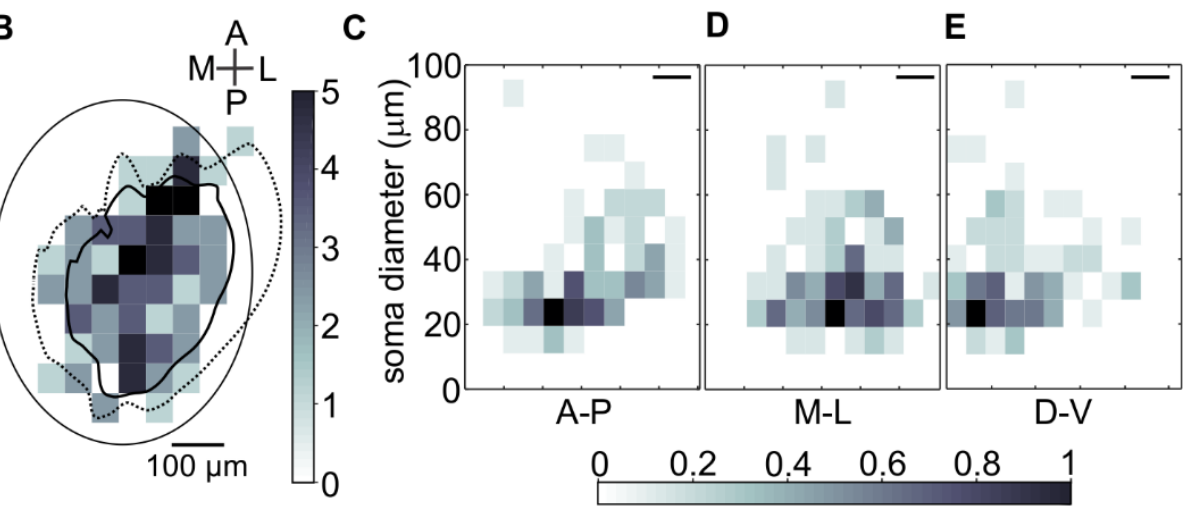

F

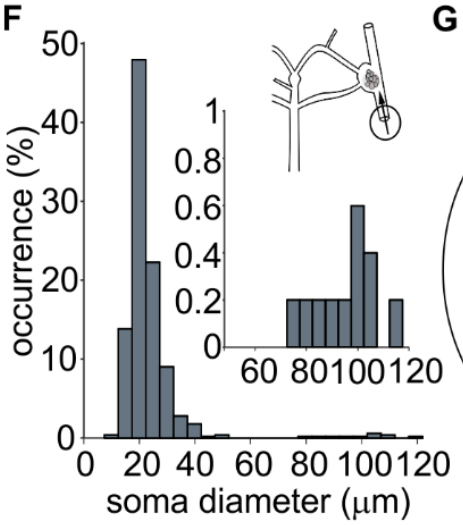

G

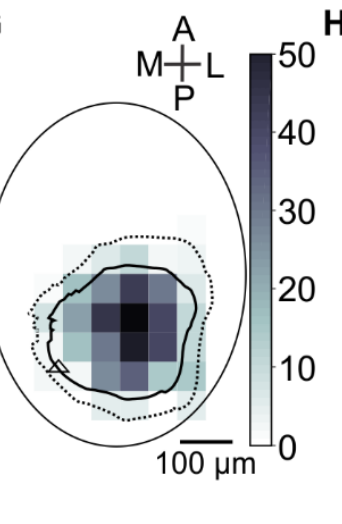

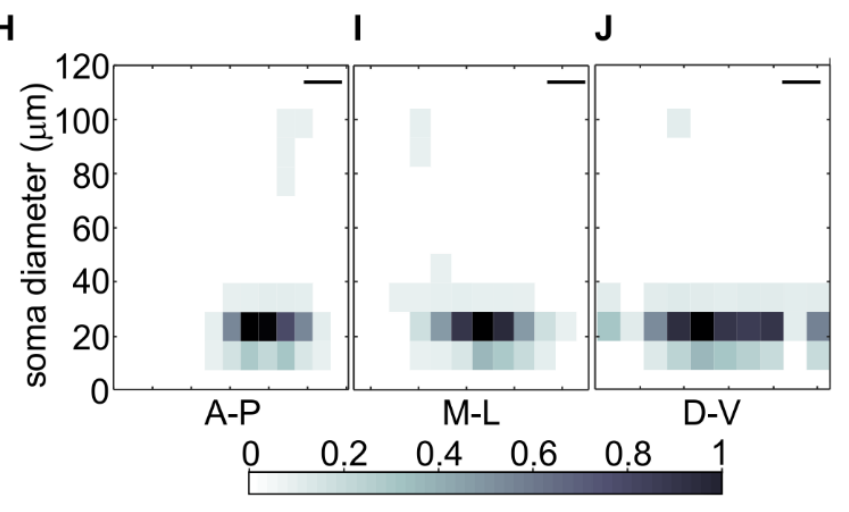

Fig. 6. Soma distribution of CoG neurons projecting to the thoracic ganglion (TG) and brain. A: Histogram of soma diameters for neurons projecting to the $T G(N=4)$. Inset: backfill location. B: 2D density plot showing the distribution of soma locations projecting to the TG. Number of somata in each bin is as indicated by the shaded bar (bin size, $50 \times 50 \mu \mathrm{m}, \mathrm{N}=4$ ). Average contour (solid line) and corresponding standard deviation (dashed line) contours are overlaid on each plot. C-E: Density plots showing the preferred locations of TG cluster somata with different diameters along the A-P (C), M-L $(D)$, and D-V (E) axes. Different shades of the bar on the right correspond to normalized density of somata for C- E. F: Histogram of soma diameters for neurons projecting to the brain $(\mathrm{N}=11)$. Inset top right: backfill location. Inset lower right: details for the larger diameter somata $(>50 \mu \mathrm{m})$. G: 2D density plot showing the distribution of soma locations of neurons projecting to the brain. The shaded bar gives the number of somata per bin (bin size, $50 \times 50 \mu \mathrm{m}, \mathrm{N}=11$ ). Average contour (solid line) and corresponding standard deviation (dashed line) are overlaid on the distribution. The triangle shows the average location of the L-cell. H-J: Density plots showing the preferred locations of neurons projecting to the brain with different diameters along the A-P $(H), M-L(I)$, and D-V $(J)$ axes. Different shades correspond to normalized density of somata for $\mathrm{H}, \mathrm{I}$ and J. Scale bars, $100 \mu \mathrm{m}(\mathrm{C}, \mathrm{D}, \mathrm{H}, \mathrm{I})$ and $20 \mu \mathrm{m}(\mathrm{E}, \mathrm{J})$. 


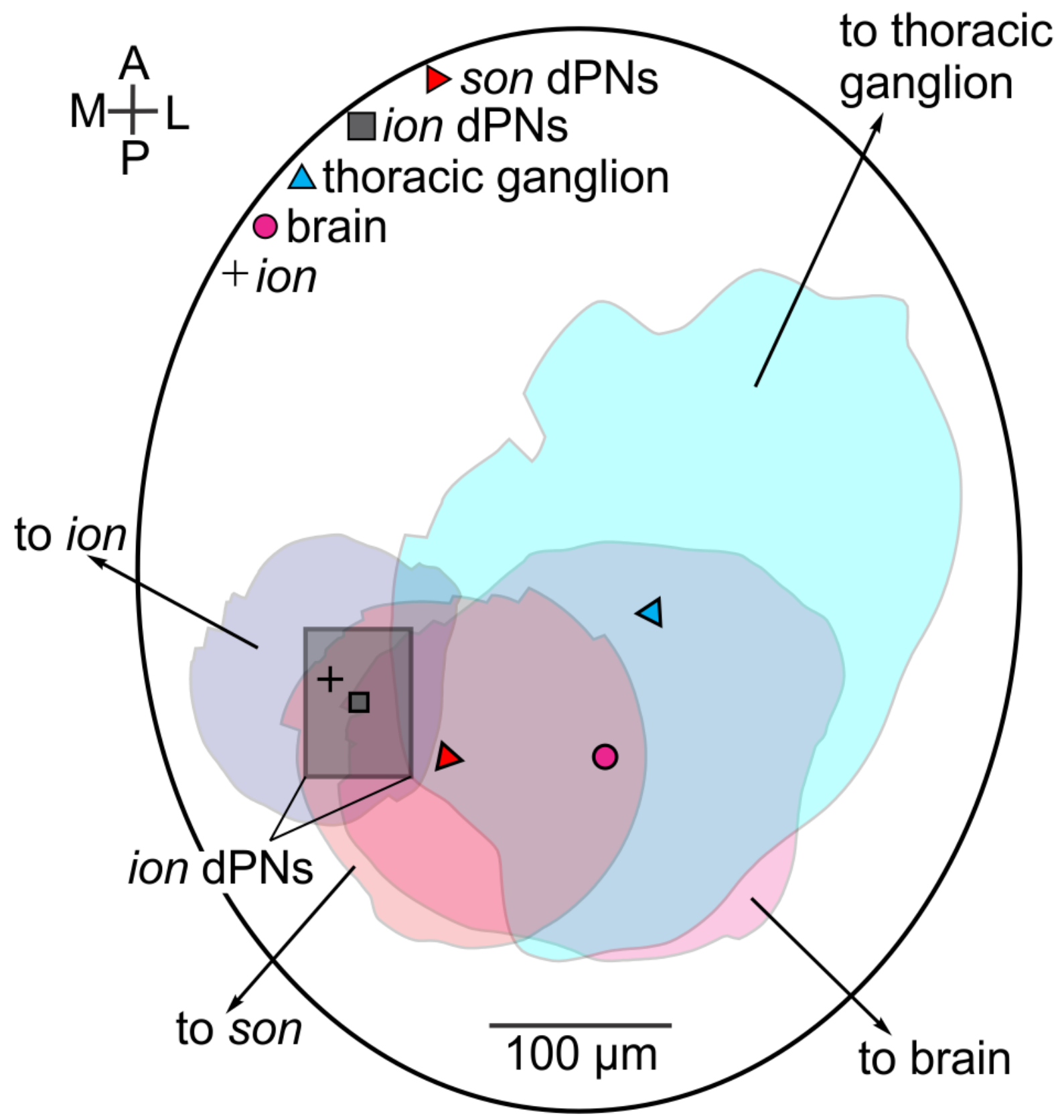

Fig. 7. CoG neurons projecting via different pathways overlap in their locations, but have distinct spatial preferences. Averaged contours from all pathways studied are arranged on the same plot as labeled, with the respective centroids indicated by the symbols: son projecting dPNs $(\boldsymbol{\nabla})$, ion projecting $\mathrm{dPNs}(\boldsymbol{\varpi})$, all ion projecting CoG neurons $(+)$, thoracic ganglion $(\boldsymbol{\Lambda})$ and brain $(\bullet)$. 
A
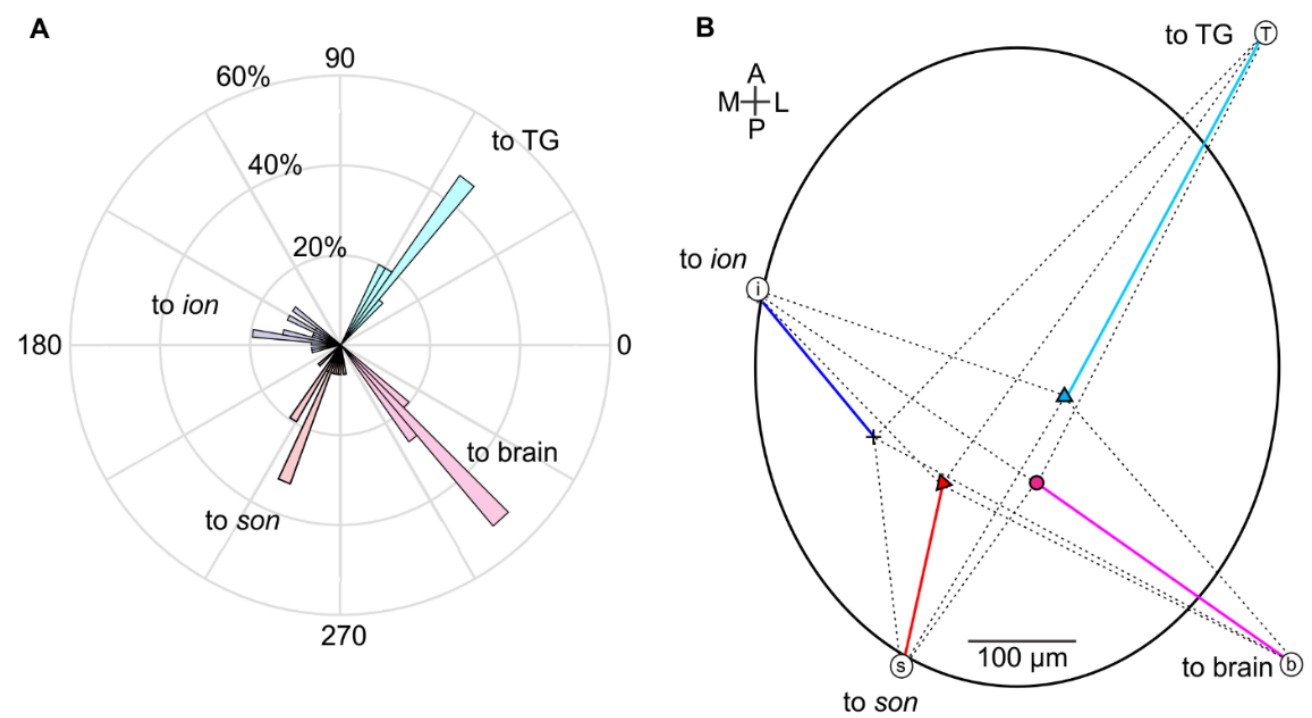

C

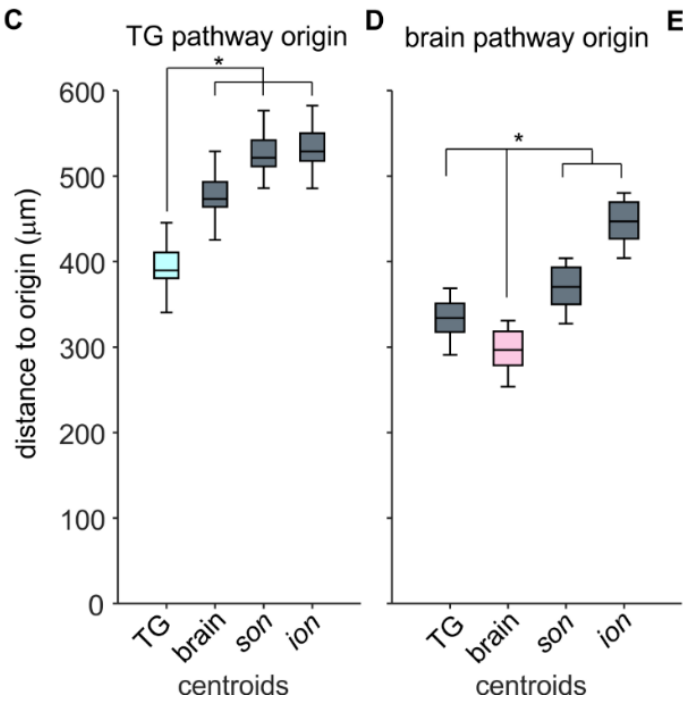

B ion pathway origin

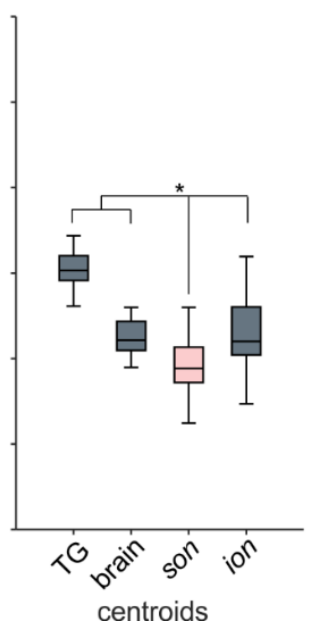

Fig. 8. Soma cluster centroids are spatially weighted towards their respective pathway origins. A: Normalized angular distribution of nerve pathway origins relative to the center of the average ganglion (bin size 5 degrees, plot radius corresponds to occurrence (\%), $\mathrm{N}=15$ ). B: Average location of nerve pathway origins (open circles (o) with respective pathway initial) and soma cluster centroids (son $(\boldsymbol{\nabla})$, ion $(+)$, TG $(\mathbf{\Delta})$ and brain $(\bullet))$. The shortest distance between a given nerve pathway origin and each soma cluster centroid is shown as a solid line and in color, longer distances as grey dashed lines. C-F: Comparisons of distance measurements from each soma cluster centroid to each pathway origin. The most proximally located soma cluster to a given pathway origin was always the cluster whose axons project through that pathway (TG projection pathway origin: $(H(3)=43.552$, $p<0.001$; brain projection pathway origin: $(H(3)=48.240, p<0.001$; son dPNs projection pathway origin: $(\mathrm{H}(3)=34.835, \mathrm{p}<0.001$; ion $\mathrm{dPN}$ s projection pathway origin: $(\mathrm{H}(3)=31.104$, $\mathrm{p}<0.001$; all shown pairwise comparisons $\mathrm{p}<0.05$ (Kruskal-Wallis one-way analysis of variance on ranks). 
A

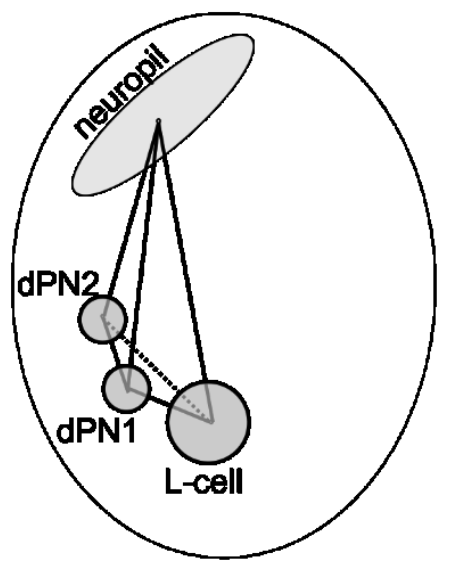

D

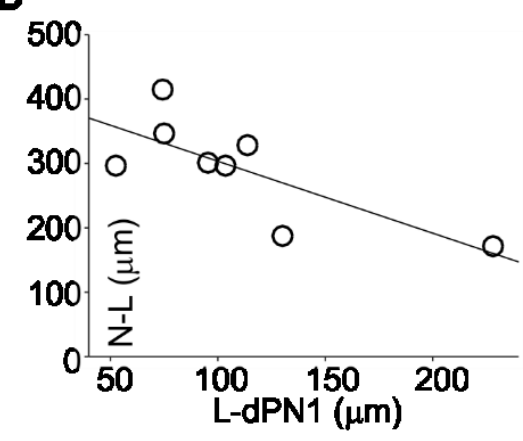

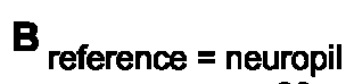

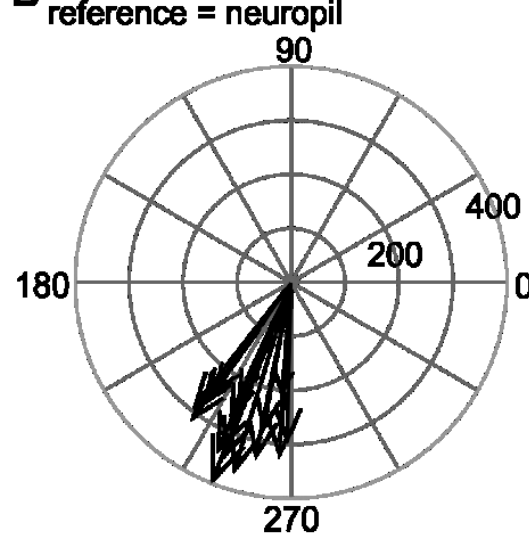

$\mathbf{E}$

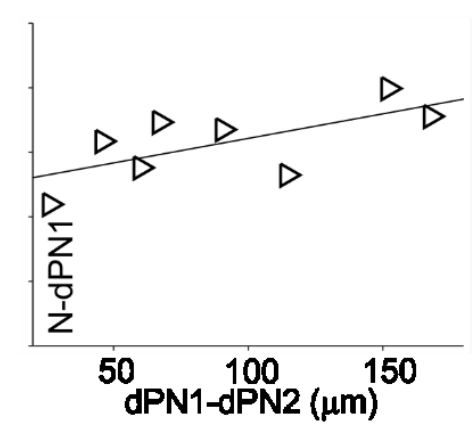

C $_{\text {reference }}=$ L-cell

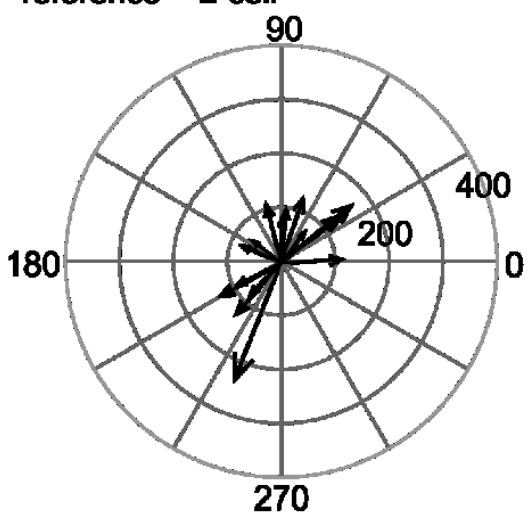

$\mathbf{F}$

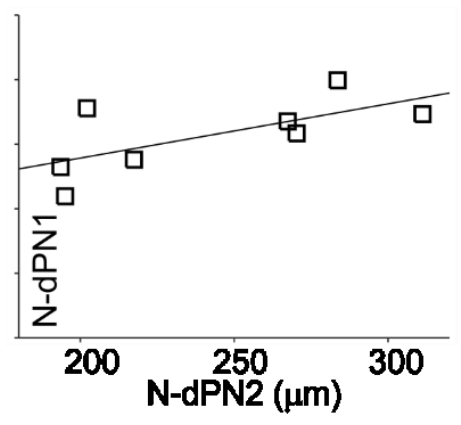

Fig. 9. Variability of dPNs location within the CoG. A: Schematic representation of the positions of ion dPN1 and ion dPN2 with respect to the L-cell and the neuropil. B: Vector angles and distances for both ion dPNs in relation to the neuropil. C: Vector angles and distances for both ion $\mathrm{dPNs}$ in relation to the L-cell. D: The distance between the neuropil and the L-cell was negatively correlated with the distance between L-cell and ion dPN1 $(R=-0.75, p<0.03)$. E: The distance between the neuropil and ion dPN1 was positively correlated to the ion dPN1 and ion dPN2, although not significant ( $R=0.671$ and $p=0.069)$. $F$ : The distance between the neuropil and ion $d P N 1$ was positively correlated to the neuropil and ion $\mathrm{dPN} 2$, although not significant $(\mathrm{R}=0.672$ and $p=0.068$ ). 
CHAPTER III: A PREMOTOR NETWORK ENCODES MULTIMODAL INFORMATION AND CONTROLS MOTOR OUTPUT VIA A COMBINATORIAL CODE

\section{Abstract}

A ubiquitous feature of the nervous system is the quick processing of simultaneously arriving sensory inputs from different modalities. While also a pivotal task for executing proper behaviors, the mechanisms neural networks use to encode distinct sensory modalities and to control motor activity remain little understood. We studied the role of premotor network activity in multimodal information encoding and motor output control in the crustacean stomatogastric nervous system. Using multi-neuron imaging of the commissural ganglion premotor region, we found that chemosensory and mechanosensory modalities were represented by overlapping, yet distinct neuronal populations (network participation), with different proportions of excited and inhibited neurons (network activation). Each modality elicited functionally different variants of the downstream pyloric motor pattern. Simultaneous (bimodal) sensory stimulation was represented by a distinct combination of premotor neurons across the network. Independent of sensory condition, though, both network activation and participation predicted changes in the pyloric output observed, demonstrating that a combinatorial code of premotor network activation and participation is used for sensory modality distinction and control of motor output.

\section{Introduction}

Integrating information from multiple sensory modalities and producing appropriate motor outputs are vital functions of the nervous system, and the neural networks underlying these two functions are tightly linked in both vertebrates and invertebrates. 
From a traditional perspective, individual senses are first integrated separately and subsequently combined at numerous multimodal convergence zones, including cortical and subcortical regions (Ghazanfar and Schroeder, 2006), as well as multimodal association areas (Rauschecker et al., 1995; Ehmer and Gronenberg, 2002; Schroeder and Foxe, 2005). More recent observations of multimodal responses in once-considered modality-specific regions, however, suggest that multimodal interactions also occur at earlier stages of sensory processing (Fu et al., 2003; Hackett and Schroeder, 2009). There is also increasing behavioral and physiological evidence from single-cell, tracing, and neuroimaging studies supporting multimodal processing as a ubiquitous function of the nervous system, rather than as a localized feature (Driver and Noesselt, 2008).

While responses to individual sensory modalities are often well-characterized, little is known about how networks of neurons process multimodal information, and how this effectively leads to behavioral responses. Multimodal interactions occur in upstream motor control circuits (Reig and Silberberg, 2014). Convergence of multimodal information has also been shown in downstream premotor circuits such as the brainstem and spinal cord (Juvin et al., 2016; Kardamakis et al., 2016), and the insect thoracic ganglia (Burrows and Pflüger, 1988; Stein and Schmitz, 1999; Stein et al., 2006) that directly initiate and modulate behavioral actions (Getting and Dekin, 1985; Meredith and Stein, 1985; Kozlov et al., 2009). Several hypotheses exist for the encoding of multimodal information by neural networks, which typically involve distinct neuronal firing rates (e.g. rate code) (Koulakov et al., 2007; Pennartz, 2009) or patterns of neuronal activation and participation (e.g. combinatorial code) (Osborne et al., 2008; Rohe and Noppeney, 2016) across the network(s) to explain how different modalities 
are represented. How such multimodal information is translated for the control of motor output remains ambiguous.

Small motor circuits, such as central pattern generators, are particularly useful in the study of mechanisms for control and modulation of behavioral output (Nusbaum and Beenhakker, 2002; Kozlov et al., 2009; Selverston, 2010). These well-characterized multifunctional building blocks of rhythmic behaviors are found in both vertebrates and invertebrates (Calabrese and De Schutter, 1992; Kiehn, 2006; Briggman and Kristan, 2008; Katz, 2016), and can produce a variety of distinct outputs in different sensory and modulatory conditions (Xin et al., 1996; Marder and Thirumalai, 2002; Berkowitz et al., 2010; Harris-Warrick and Johnson, 2010). Their activity is controlled by descending modulatory projection neurons that are embedded in upstream premotor networks (Norris et al., 1996; Nusbaum and Blitz, 2012; Daghfous et al., 2016; Yeh et al., 2017). These premotor networks integrate sensory information from a variety of modalities, and relay the integrated information to the motor circuits via the descending projection neurons that control behaviorally relevant and functionally distinct motor acts (Kiehn, 2006; Le Ray et al., 2011; Schnell et al., 2017; Sen et al., 2017), and continuously modulate ongoing behaviors (Nusbaum and Blitz, 2012; Kozlov et al., 2014). The direct link between multimodal encoding and motor control in premotor networks provides a means to investigate multimodal encoding in the immediate context of behavioral output, and may provide a model for more complex multimodal brain areas further upstream.

It is well established that sensory pathways activate multiple descending projection neurons simultaneously, and that this has functional consequences for 
downstream motor output, like switching from ingestion to egestion in Aplysia feeding (Morgan et al., 2002), and forward to backward walking in Drosophila (Sen et al., 2017). Nonetheless, it is unknown how premotor networks, in which the projection neurons are embedded, respond to and encode multimodal information. In the crustacean stomatogastric nervous system, a small number of descending projection neurons participate in a premotor network that resides within the paired commissural ganglia (CoGs), each of which contain less than 220 neurons (Follmann et al., 2017)), and process information from different sensory modalities (Blitz et al., 2004; Hedrich et al., 2009; Stein, 2009). We used these advantageous features to investigate the coding mechanism for multiple sensory modalities presented both individually (unimodal inputs) and simultaneously (bimodal input). Specifically, we selectively activate the chemosensory inferior ventricular neurons (IVs) and the mechanosensory ventral cardiac neurons (VCNs) with physiologically-relevant stimulation parameters (Beenhakker et al., 2004; Hedrich and Stein, 2008). Both modalities elicit functionally distinct motor patterns in the downstream central pattern generators of the stomatogastric ganglion (STG), via their actions in the CoG premotor network (Blitz et al., 2004; Hedrich et al., 2009). Each sensory modality causes a distinct response in a small set of identified descending projection neurons (Beenhakker and Nusbaum, 2004; Hedrich et al., 2009; Stein, 2009), inducing modulatory effects on both the pyloric (filtering food) and gastric mill (chewing) rhythms in the STG (Sigvardt and Mulloney, 1982; Cazalets et al., 1987; Blitz et al., 2004). While individual descending projection neurons can act as command-like neurons and alter motor output via their firing patterns (Nusbaum et al., 1992), sensory stimulation always recruits multiple projection 
neurons (Hedrich et al., 2009). Moreover, selective manipulation of the few identified projection neurons cannot recreate all aspects of the observed sensory-induced motor pattern changes (Hedrich et al., 2009), suggesting that additional portions of the network play an important role in mediating the sensorimotor effects.

Using multi-neuron imaging with a voltage-sensitive fluorescent dye, we show that the chemosensory and mechanosensory modalities are represented by distinct premotor network responses. Primarily, these differences were exhibited in the activation, i.e. the difference in the proportion of excited and inhibited neurons, and the participation (the specific neurons involved) of the premotor network. Bimodal input was encoded by a set of neurons distinct from either unimodal condition. Independently of sensory condition, however, changes in network activation and participation were correlated with several parameters of the pyloric output. Our findings support a premotor combinatorial code for multimodal representation and motor pattern control: one in which network activation and participation are distinct between sensory modalities, and both of these network measures relate to changes in downstream motor output.

\section{Methods}

Animals

Adult Jonah crabs (Cancer borealis) were acquired from The Fresh Lobster Company (Boston, MA) or Ocean Resources Inc. (Sedgwick, ME). Crabs were kept in tanks with artificial seawater (salt content $\sim 1.025 \mathrm{~g} / \mathrm{cm}^{3}$; Instant Ocean Sea Salt Mix, Blacksburg, VA), at a temperature of $11^{\circ} \mathrm{C}$ and a 12-hour light-dark cycle. Animals were 
anesthetized on ice for 30 minutes before dissection. We used isolated nervous systems to perform all of our experiments (Blitz and Nusbaum, 1997).

\section{Solutions and reagents}

For electrophysiological experimentation, nervous systems were continuously superfused $(7-12 \mathrm{ml} / \mathrm{min})$ with chilled $\left(10-13^{\circ} \mathrm{C}\right) \mathrm{C}$. borealis saline, consisting of (all from Sigma, St. Louis, MO): $\mathrm{NaCl}, 440 \mathrm{mM} ; \mathrm{KCl}, 11 \mathrm{mM} ; \mathrm{MgCl}_{2}{ }^{*} 6 \mathrm{H}_{2} 0,26 \mathrm{mM} ; \mathrm{CaCl}_{2}, 13$ $\mathrm{mM}$; trisma base, $10 \mathrm{mM}$; maleic acid, $5 \mathrm{mM}(\mathrm{pH}$ 7.4-7.6).

Anterograde tracing of sensory pathways

A petroleum jelly well was constructed around inferior ventricular (ivn) and dorsal posterior esophageal (dpon) nerves that contained the IV and VCN axons, respectively. Saline was removed from each well and replaced with filtrated water. The nerves were then transected, and wells filled with dye (for ivn well: $8 \%$ nickel(II) chloride; dpon well: cobalt(II) chloride and nickel(II) chloride (5:2; 5\%-8\%; Sigma, St. Louis, MO). The preparation was then incubated at $4{ }^{\circ} \mathrm{C}$ for $2-4$ days. Saline was supplemented with Penicillin-Streptomycin (Sigma) at $10 \mathrm{mg} / \mathrm{L}$ to prevent bacterial contamination. After incubation, the well was removed, and the dish was rinsed with saline. For precipitation, 25-30 drops of saturated dithiooximide (Sigma) solution were added. Terminals filled with nickel(II) chloride appeared dark blue (IV neurons) and the dye mixture for VCN fills appeared red.

Application of a voltage-sensitive dye

The lipophilic voltage-sensitive dye Di-4-ANEPPDHQ (Thermo Fisher Scientific) was bath-applied to stain neuronal membranes (Goldsmith et al., 2014). $5 \mathrm{mM}$ stock solutions in dimethyl sulfoxide were aliquotted for single use, and kept at $-20^{\circ} \mathrm{C}$. 
Immediately before application, solutions were diluted 1:1 with pluronic acid F-127 (20\% solution; Biotium, Hayward, CA) dimethyl sulfoxide solution, and mixed with saline to a final concentration of $50 \mu \mathrm{M}$. A petroleum jelly well was built around the desheathed CoG (fig. 1) and the dye was applied for 30-60 minutes, after which the petroleum jelly well was removed, and the preparation was continuously superfused with cooled (10-13 $\left.{ }^{\circ} \mathrm{C}\right)$ saline for the remainder of the experiment.

\section{Stimulation of sensory nerve pathways}

Mechanosensory: VCNs were activated by stimulating the dpon extracellularly with single stimulus trains of $1 \mathrm{~Hz}$ or $15 \mathrm{~Hz}$ stimulation frequency (Hedrich and Stein, 2008). Chemosensory: The IV neurons were activated by stimulating the ivn extracellularly with single stimulus trains of $1 \mathrm{~Hz}$ or $40 \mathrm{~Hz}$ stimulation frequency (Beenhakker et al., 2004). Petroleum jelly wells were built around the dpon and the ivn. For each nerve, one of two stainless-steel stimulation electrodes was placed inside the petroleum jelly compartment, and the other was placed outside. Stimulation commands were generated in Spike2 (version 7.13; Cambridge Electronic Design) and converted to analog signals via a Power 1401 digital-analog converter (Cambridge Electronic Design).

\section{Optical imaging}

For recording fluorescence changes the MiCam02 imaging system and BV-ANA software (Brain-Vision Analyzer, Version 11.08.20; SciMedia Ltd, Tokyo, Japan) were used (spatial resolution=192×128 pixels; frame rate=250 frames/second). Individual recordings lasted 20 seconds and were repeated many times in a given experiment. Excitation light was provided by a narrowband LED with $525 \mathrm{~nm}$ (CoolLED, Yorktown Heights, NY), and fluorescence emission was filtered through a quadband filter cube 
(Semrock, Inc., Rochester, NY). Excitation light intensities varied and were adjusted to the individual preparation. We used a 20x objective (UMPlanFL N, NA 0.30, WD 3.3 mm, cc=water; Olympus Corporation, Tokyo, Japan) mounted on an upright epifluorescence microscope (modified BX51, Scientifica, East Sussex, UK).

\section{Extracellular nerve recordings}

Spiking activities of the pyloric neurons were acquired via extracellular recordings of motor nerves posterior to the STG (fig. 1A, schematic): we used the lateral ventricular nerve (Ivn) for LP activity, the pyloric dilator nerve ( $p d n)$ for PD activity, and the pyloric constrictor nerve (pyn) for PY activity. We used petroleum jelly wells and subsequent measurements of field potential changes between two stainless steel wires (one inside and one outside of each well) to extracellularly record action potentials. The differential signal was recorded, filtered, and amplified with an AC differential amplifier (A-M Systems Modell 1700, Carlsborg, WA, USA). Files were recorded, saved and analyzed using Spike2 Software (version 7.11; CED, Cambridge, UK).

Data analysis and figure construction Imaging and electrophysiological data were analyzed with the Brain-Vision Analyzer software (BVAna SciMedia Ltd, Tokyo, Japan), Spike2 (version 7.13; CED) and custommade MATLAB (version R2014b, MathWorks) scripts. Final figures were prepared with MATLAB and CorelDraw (version X7 for Windows, http://www.coreldraw.com). For spreadsheet analysis, Excel (version 2010-2013 for Windows, Microsoft) and R (version 3.3.1, The R Foundation for Statistical Computing) were used. Data are given as mean \pm SD unless otherwise noted. "N" denotes the number of ganglia used; " $n$ " denotes the number of neurons or trials. Significance is indicated using ${ }^{*}(p<0.05) ;{ }^{* *}(p<0.01)$; 
$\left.{ }^{* * *}(p<0.001)\right)$. Statistical tests were paired t-test, Pearson correlation coefficient, twosample Kolmogorov-Smirnov (KS) test, Tukey-Kramer test in conjunction with one-way ANOVA. Details of each individual test used can be found in the figure legends to which they pertain.

Imaging analysis

For comparing changes in fluorescence of the entire imaged region (spatial averaging) of the different sensory inputs stimulation (fig. 2C), we used the average whole field change in fluorescence during stimulation and pre-stimulation. This included the averaged signals of all imaged neurons.

We measured the action potential ('spike' or 'firing') frequencies of all imaged neurons to detect sensory-induced responses in CoG neurons. Voltage-sensitive dyes are sufficiently fast to detect sub-millisecond events (Obaid et al., 2004). Individual cell traces and contours were extracted using BV-ANA software, and further analysis was executed in MATLAB. Traces were processed with a drift removal based on six order polynomial fit, and spike threshold was based on the median of the signal (Quiroga et al., 2004).

For rate coding analyses (fig. 2E), we calculated in each experiment for each condition, the ratio between spike frequency during stimulation (stimON) and immediately preceding stimulation (stimPRE). For each condition, the distribution of the frequency ratios of all neurons in a given preparation was plotted as a histogram (Bin size: 0.1). Distributions were normalized to the maximum count for each experiment. Frequency ratio distributions were then averaged across preparations, and tested for differences between conditions using the Kolmogorov-Smirnov (K-S) test. Ratios>1 indicate 
neuronal activity increases ('excitation'), ratios $<1$ indicate activity decreases ('inhibition'), and ratios $=1$ indicate no change in spike frequency. Ratios were used to categorize individual neurons as "excited", "inhibited", or "non-responsive" for analysis later (fig. 5C-G). Pairwise comparisons for these categorizations were made between IV and VCN conditions for each CoG, and subsequently averaged across ganglia.

Neurons were defined as unimodal when their activities changed in response to stimulation of only one of the two sensory modalities. Neurons that responded to both sensory modalities were defined as multimodal. Neurons that did not respond to either stimulation were defined as "non-participant" (fig. 5A).

For the spatial distribution analysis (fig. 6), we calculated the angle from the Cartesian coordinates and used the angular variance as a measure of directional variability. To normalize the distances for each ganglion, we determined a polygon contour around the outer neurons, found the centroid, and calculated the ratio between the distance from neuron to centroid and distance from border to centroid, $\mathrm{dratio}_{\mathrm{i}}=\mathrm{d}($ neuron, centroid $) / \mathrm{d}($ border, centroid $)$.

For premotor network participation (see fig. 7E and fig. 9), we obtained the proportion of premotor neurons that share a similar response type to two of the sensory conditions, while the response to the remaining condition differs. For example, a neuron that was excited by IV stimulation, but inhibited by both VCN and bimodal stimulations would be a unique participant for the IV condition, while a neuron that was excited by IV, VCN, and bimodal stimulations is not a unique participant and was omitted from the analysis. 
Premotor network activation (see fig. 7D and fig. 10) was calculated as the difference between the proportion of excited neurons and inhibited neurons. If network activation is positive, then a larger proportion of neurons were excited (a net excitation) by a given sensory condition; if network activation is negative, then a larger proportion of neurons were inhibited by a given sensory condition (a net inhibition).

For the expected map responses (congruency under bimodal conditions, fig. 11), we considered nine response combination scenarios given the three potential neuronal responses (excited, inhibited, and non-responsive) to unimodal sensory input. Seven of these can be used to generate clear expectations of bimodal stimulation effects and to test whether neuronal responses to bimodal stimulation are the additive result of the responses observed during unimodal stimulation: excitatory responses to both unimodal inputs will summate to an excitatory bimodal response (IVex $\left./ \mathrm{VCN}_{\mathrm{ex}}=\mathrm{CO}_{\mathrm{ex}}\right)$. An inhibitory response to both unimodal inputs will yield an inhibited bimodal response $\left(\mathrm{IV}\right.$ inh $\left./ V N_{\text {inh }}=\mathrm{CO}_{\text {inh }}\right)$, and no response to either stimulus should not result in a response to bimodal stimulation (IVNR/VCNNR=CONR). An excitatory response to one unimodal input and no response to the other will summate to an excitatory response for bimodal stimulation ( $\left(\mathrm{V}_{\mathrm{ex}} / \mathrm{VCN}_{\mathrm{NR}}=\mathrm{CO}_{\mathrm{ex}}\right.$; IV $\left.\mathrm{VNR}_{\mathrm{NR}} / \mathrm{VCN}_{\mathrm{ex}}=\mathrm{CO}_{\mathrm{ex}}\right)$, and an inhibitory response to one input with no response to the other will yield an inhibitory bimodal response $\left(I V_{\text {inh }} / V_{C N}=C_{\text {inh }}\right.$; IV $\left.V_{N R} / V C N_{\text {inh }}=C_{\text {inh }}\right)$. Two scenarios $\left(I V_{\text {ex }} / V C_{\text {inh }}\right.$ and $I V_{\text {inh }} / V_{C N}$ ex $)$ are inconclusive, because the additive outcome cannot directly be predicted as it depends on the weighting of excitation and inhibition. We used spatial maps of the previously characterized neuronal responses to create spatial maps of the expected outcomes (fig. 11). A Boolean-based comparison of the additive expectations with those 
data acquired for multimodal responses was used to demonstrate the proportion of the network that did not respond additively ("no match").

Motor pattern analysis

For the motor neuron analysis a custom-written program for Spike2 was used to determine pyloric rhythm activities. Pyloric neuron activity measurements were calculated as follows: firing frequency as the number of action potentials spikes divided by the interval of time (s) measured; burst duration as the time (s) from the first to the last spike in a burst; spikes per burst as the number of spikes counted in a burst; intraburst firing frequency as the number of spikes in a burst (minus 1) divided by the burst duration (s); and the cycle period was determined by calculating period between the onset of 2 successive pyloric dilator (PD) neuron bursts. Measurements were plotted as the normalized change (norm. $\Delta$ ) between stimPRE and stimPOST (fig. 8). These same measures were used in the correlation analyses with premotor network activation (fig. 7).

For pyloric motor output uniqueness measures, we calculated the absolute difference in the normalized change between two sensory conditions. These were then plotted against the corresponding similarities in network participation between two conditions (fig. 6).

\section{Results}

The CoG premotor region processes information from multiple sensory modalities The chemosensory (IV) and mechanosensory (VCN) pathways are known to modulate downstream STG motor patterns, and these effects are mediated by the CoG premotor network (Beenhakker and Nusbaum, 2004; Hedrich et al., 2009; Stein, 2009). Despite 
this, no direct anatomical evidence for a convergence of both sensory pathways in the CoGs exists. We first traced IV and VCN axon projections to the CoG using backfill stainings (see Materials and Methods) to determine whether neurons from both pathways arborize in the CoG. Backfills of the VCN pathway were started at the dorsal posterior esophageal nerve (dpon, fig. 1), through which the VCNs innervate the CoG (Beenhakker et al., 2004). IV neuron backfills were carried out via the inferior ventricular nerve (ivn, fig. 1), through which the two IV neuron axons project from the brain to the stomatogastric nervous system (Christie et al., 2004). As previously suggested by electrophysiological data (Beenhakker et al., 2004; Christie et al., 2004; Hedrich and Stein, 2008), IV fibers entered the CoG via the inferior esophageal nerve (ion) and the superior esophageal nerve (son), and the VCN fibers entered via the son. The neurites of both pathways terminated in the neuropil of the CoG (fig. $2 \mathrm{~A}$, dotted area), a region associated with overlapping innervation from other sensory and modulatory pathways, as well as from ascending thoracic and descending brain fibers (Wiersma, 1957; Kirby and Nusbaum, 2007; Follmann et al., 2017). Despite morphological variation in CoG size and shape (see (Follmann et al., 2017)), IV and VCN fibers consistently overlapped within the neuropil $(\mathrm{N}=6)$.

Previous studies demonstrated that IV and VCN pathways influence the activities of the same four identified descending premotor neurons (Blitz et al., 2004; Hedrich et al., 2009). While some monosynaptic connections have been identified, most sensory effects on the premotor neurons and the downstream motor patterns are due to polysynaptic connectivity and in response to longer-lasting and continuous stimuli (Beenhakker et al., 2004; Hedrich et al., 2009). This suggests that the activity of the 
local premotor network is essential in mediating the collective premotor and motor responses. To describe the sensory response of the CoG premotor region, we employed multi-neuron imaging with fast voltage-sensitive dyes, while selectively stimulating IV and VCN pathways (fig. 1 for schematic). A key advantage of this technique is the combination of high spatial and temporal resolution (Goldsmith et al., 2014). The cell bodies of premotor neurons innervating the STG are restricted towards the medial-posterior region of the CoG (Follmann et al., 2017). We thus focused on this region (fig. $2 \mathrm{~A}$ and fig. 1, dashed rectangles), and averaged the optical signals of all cell bodies in this area for an initial assessment of the premotor network response (fig. 2B). Both IV and VCN modalities induced changes in fluorescence over the course of the stimulus train (IV=40 Hz stimulation frequency; $V C N=15 \mathrm{~Hz}$ stimulation frequency; fig. 2C), reflecting the change of activity within the premotor network in response to each sensory stimulation. The magnitude of the VCN-induced response was consistently greater than that elicited by IV stimulation ( $N=12, p<0.05$, paired t-test; fig. $2 \mathrm{D})$, suggesting that IV and VCN modalities are processed differently by the CoG premotor network.

To test whether these differences were indicative of a distinct recruitment of neuronal sub-populations, or rather of different neuronal response intensities within the same population (or combinations thereof), we examined CoG network activity with single-neuron resolution. Fig. 3 compares the single-neuron activity of a CoG using simultaneous intracellular and optical recordings (raw traces in fig. 3A). Waveform averages were similar between optical and intracellular recordings at both high and low spike frequencies (fig. 3B). Only 6 out of the 114 spikes recorded in this analysis were 
mis-detected in the optical recording (95\% accuracy, fig. 3C), reaffirming the reliability of VSD imaging in representing neuronal activity (see Preuss et al., 2013). Using a low frequency $(1 \mathrm{~Hz})$ stimulation protocol to test for short-latency connections, we found neurons that were reliably excited during only IV or VCN stimulation, and some neurons that were excited by both modalities (stimulated at different times, examples shown in fig. 4A). There were no sensory-specific differences in mean CoG neuron response latency (fig. 4B, $N=11, p=0.19$, unpaired two-sample t-test), and no difference in the distribution of latencies across all CoG neurons $(N=11, n=129$ neurons, $p=0.14$, twosample Kolmogorov-Smirnov test; fig. 4B). However, on average, fewer neurons were excited by IV stimulation ( $4.2 \pm 2.3$ neurons/ganglion) than by VCN stimulation $(7.5 \pm$ 5.6 neurons/ganglion; $N=11$, fig. $4 \mathrm{C}$ ), complementing the results from the averaged network responses above (fig. 2D).

When we used physiologically relevant stimulation frequencies (a single 6second train of either $40 \mathrm{~Hz}$ (IV) or $15 \mathrm{~Hz}$ (VCN) (Beenhakker et al., 2004; Hedrich and Stein, 2008)), significantly more neurons were excited by either stimulation (IV: $34.9 \pm 7.1$ neurons/ganglion; VCN: $39.3 \pm 9.5$ neurons/ganglion; $N=11, p<0.001$, paired t-test; fig. 4C), suggesting that polysynaptic interactions in the local premotor network contribute to sensory processing. Fig. 2D shows non-averaged traces of eight neurons from the same preparation that responded to one or both sensory modalities (see also fig. 1D). Importantly, some neurons were differentially affected by both modalities. For example, neuron 6 was unaffected by IV stimulation, but excited during stimulation of the VCN pathway. Neurons 4 and 5 were excited by both modalities, but with different firing frequencies, and neuron 3 was inhibited by VCN stimulation (fig. 4D, asterisk). These 
findings are consistent with a premotor combinatorial coding scheme, but thus far cannot refute the additional use of a network rate code.

Similarities in the premotor spike frequency distributions between modalities contradict a network rate code

While rate codes exist for unisensory parameter distinction (Wang et al., 2008), it is unclear how encoding occurs when the same network processes more than one sensory modality. A rate coding mechanism would predict that the same CoG neurons are recruited by IV and VCN stimulation, with the distinguishing information between modalities being encoded in the firing frequency responses of these neurons. For a rate code to exist, the CoG premotor network distribution of firing responses should thus differ between modalities. Across all conditions, neurons, and ganglia, CoG neuron firing frequencies ranged from 0.33 to $14 \mathrm{~Hz}(\mathrm{~N}=12)$. Most $\mathrm{CoG}$ neurons were spontaneously active even in the absence of sensory stimuli, at rates of $0.33-6.8 \mathrm{~Hz}$ $(\mathrm{N}=12)$. When we compared the range of firing frequencies associated with IV and VCN conditions, we found no differences: $0.33-14.00 \mathrm{~Hz}$ (IV) and 0.33-13.80 Hz (VCN). More importantly, there was no difference in the distribution of firing frequency changes induced by each condition. Fig. 4E shows the distribution of normalized frequency changes for both pathways, using the ratio of CoG neuron firing frequency (frequency ratio) during stimulation (stimoN) to the frequency preceding stimulus presentation (stim $P R E$, compare fig. 1). Over the entire network ( $N=12, n=878$ neurons), there was no difference in this distribution ( $N=12, p=0.144, K S$ test; fig. $4 \mathrm{E})$, indicating that no distinct firing frequencies and thus no network rate code exists for distinguishing between sensory modalities. 
Modality-specific neurons and distinct premotor network activation support a combinatorial code for multimodal representation

Most premotor neurons (>98\%) participated in sensory processing, and less than $2 \%$ did not respond to stimulation of either pathway (fig. 5A,B). The large number of participating neurons supports the idea that most of the premotor network contributes to sensory encoding, but also suggests overlap of the circuits processing each input. Indeed, the majority of neurons (about $80 \%$, fig. 5B) were multimodal responders, i.e. they responded (with excitation or inhibition) to both IV and VCN stimulation. About $18 \%$ of the neurons were unimodal responders (fig. 5B), responding to only one of the two pathways. Fig. 5A shows the spatial map of unimodal and multimodal neurons from one CoG. Across ganglia, we consistently found a larger proportion of multimodal than unimodal neurons (fig. $5 B ; N=12, F(2,22)=5075.48$, $p<0.001$, repeated measures ANOVA, Tukey-Kramer post-hoc test). Moreover, the variability associated with these proportions was low across preparations, i.e. there was a consistent subset of neurons that were specific to only one or the other modality despite the high degree of overlap in the CoG neurons responding to each modality.

Up to this point, we deemed a neuron responsive to IV and VCN stimulation if it deviated from its control firing frequency (stimPRE), but the categorical responses (excitation versus inhibition) were not specifically analyzed. If a combinatorial encoding scheme was present, the proportions of excited and inhibited neurons should differ between modalities. Specifically, if multimodal neurons exhibited categorically different responses (excitation versus inhibition) between modalities, this would provide a means for distinctive representation of sensory information on top of the unique participation 
already demonstrated by the presence of unimodal responders. To determine whether such differences in neuronal activity were present, we categorized neurons as "excited" or "inhibited", depending on whether their firing frequencies increased or decreased, respectively, in response to IV and VCN stimulation. We generated activity maps of neurons that were excited, inhibited, or "non-responsive" (no change in firing frequency) to IV (fig. 5C, left) and VCN (fig. 5C, right) stimulation. First, we observed no significant spatial clustering, a feature often observed in larger brain areas (Petersen and Sakmann, 2001; Benedek et al., 2004; Venezia et al., 2017), of excited or inhibited neurons in any sensory condition (fig. 6), indicating a distributed sensory-processing throughout the whole premotor network. Neuronal responses, however, were clearly distinct: more neurons per ganglion were excited by VCN stimulation than by IV stimulation ( $\mathrm{VCN}=49.8 \pm 4.8 \%, \mathrm{IV}=45.7 \pm 2.7 \%, \mathrm{~N}=12, \mathrm{p}<0.05$ paired t-test; fig. $5 \mathrm{D})$, supporting our initial finding that VCN stimulation yields a higher proportion of excitation in the CoG premotor region (fig. 2F). Conversely, IV stimulation yielded a larger number of inhibited neurons per ganglion than $\mathrm{VCN}$ stimulation (IV=43.8 $\pm 3.0 \%, \mathrm{VCN}=40.3 \pm$ $3.9 \%, N=12, p<0.05$ paired t-test; fig. $5 E$ ). These proportions were also reflected in the unimodal and multimodal groups: significantly more unimodal and multimodal neurons were excited by VCN than by IV input (fig. 5F,G; $N=12, p<0.05$ each, paired t-test). IV stimulation caused inhibition of significantly more multimodal neurons than VCN stimulation $\left(\mathrm{IV} \mathrm{V}_{\text {inh }}=48.9 \pm 3.2 \% ; \mathrm{VCN}_{\text {inh }}=44.9 \pm 4.9 \%, \mathrm{~N}=12, \mathrm{p}<0.05\right.$, paired t-test $)$, accounting for the observed differences in modality-specific inhibition in fig. 5E. Thus, IV and VCN modalities recruited distinct combinations of CoG neurons by targeted excitation and inhibition within a largely overlapping set of neurons. 
The bimodal sensory condition elicits mechanosensory-like premotor network activation and participation

Sensory pathways were stimulated separately in the above experiments. During natural behaviors, however, sensory stimuli from multiple inputs may arrive concurrently. Although the IV and VCN modalities have never been examined in conjunction, they complement each other in vivo. The stomatogastric nervous system coordinates aspects of feeding in decapod crustaceans (Clemens et al., 1998a; b; Böhm et al., 2001). In this context, the IV neurons relay chemosensory information regarding potential food odor (feeding/unfed state) and the VCNs activate with stomach distension as the crab ingests food; at this time, both IV and VCN pathways are active (feeding/fed state). Only after the animal is done feeding (while still fed) will unimodal VCN activation occur (not feeding/fed state). To test whether concurrently arriving sensory stimuli are represented by a distinct premotor network response, we carried out an additional set of experiments: we stimulated IV and VCN pathways individually (unimodal input) and simultaneously (bimodal input), and quantified activity maps across all three sensory conditions (individual examples: IV unimodal input, fig. 7A; VCN unimodal input, fig. 7B; bimodal input, fig. 7C). Like the individual stimulations, bimodal stimulation yielded a spatially distributed network without clustering of excited and inhibited neurons (fig. 6A, right map; statistics shown in fig. 6D and $E$ describe the lack of excited and inhibited neuron spatial trends across conditions in terms of the soma spread from the center of the population and angular variance, respectively). We used network activation (the proportion of excited neurons minus the proportion of inhibited neurons) to describe responses of the premotor network across sensory conditions. The network activation 
associated with the VCN condition was higher than that measured in the IV condition (fig. 7D, left). Bimodal stimulation, however, yielded a similar network activation as observed in the VCN condition.

While this analysis was reflective of premotor network activation, it did not determine which neurons participated in the encoding of the different sensory conditions, i.e. whether particular neurons contributed to one, two, or all conditions (referred to here as 'participation'). To test for differences in network participation, we tracked the identities and responses of all neurons individually across sensory conditions and classified them as unique participants if they showed a distinct response in one condition. For example, a neuron was a unique participant of the IV condition if it was inhibited by IV stimulation, but excited by VCN and bimodal stimulation. This allowed us to measure the uniqueness of premotor network responses in terms of the neurons involved, and to track participation of individual neurons to sensory responses. About $30 \%$ of the neurons were unique to the IV condition, while approximately $20 \%$ were unique to the VCN condition and another $20 \%$ to the bimodal condition. Similar to what we observed for premotor network activation, the VCN and bimodal conditions were thus more similar to each other than to the IV condition, which had a significantly larger number of unique responders than the VCN and bimodal conditions (repeated measures ANOVA, ${ }^{*} \mathrm{p}<0.05, \mathrm{~N}=6$, Tukey-Kramer post-hoc test; fig. 7E).

Bimodal sensory input can be perceived as a unique condition that is distinct from the experience of its unimodal inputs (Driver and Noesselt, 2008; Noel et al., 2015; Meijer et al., 2017) . Little is known how such unique representations may be achieved in neural networks. The fact that about $20 \%$ of all premotor neurons were unique to the 
bimodal condition already indicated that part of the answer may lie in the participation of the individual neurons to the network response. To address this further, we generated a set of expectations for neuronal responses in the bimodal condition, based on the responses observed in each unimodal condition (see Materials and Methods). We then compared the observed responses to the predicted responses. For about $37 \%$ of the neurons, no prediction could be made. This was the case if, for example, a neuron was excited by VCN, but inhibited by IV. The bimodal response could thus not directly be predicted as it would depend on the level of excitation and inhibition in each condition. For the about $63 \%$ of the cells for which we were able to create predictions, we found a mix of additive and non-additive responses within the CoG premotor network during the bimodal condition. The responses in the unimodal conditions were only predictive (congruent) for $32.19 \pm 4.06 \%$ of neurons, while expectations were not predictive (incongruent) for $30.63 \pm 5.44 \%$ of neurons (fig. 11). Consequently, for about half of the neurons in which expectations could be generated, the observed outcomes did not match the expected outcomes, indicating a significant reorganization of the CoG premotor network activity when processing bimodal information. Thus, rather than a combined representation of the responses to individual IV and VCN stimuli, a new combination of responses emerged to represent the bimodal condition. CoG premotor network activation and participation contribute to downstream changes in pyloric motor output

Premotor networks such as the one studied here are involved in the selection and maintenance of downstream motor activities, shaping the dynamics of ongoing motor programs (Bruno et al., 2015; Hill et al., 2015), and may be involved in decision making 
when sensory conditions change (Briggman et al., 2005; Briggman and Kristan Jr., 2006). To test whether network participation or network activation (or both) are decisive for motor output, we monitored the activities of several identified STG motor neurons across the different sensory conditions (fig. 8, and see fig. 1A for schematic). We recorded the pyloric dilator (PD, fig. 8A) neurons, the pyloric constrictor (PY, fig. 8B) neurons, and the lateral pyloric (LP, fig. 8C) neuron. They together build the triphasic pyloric rhythm, with PD driving the rhythm as part of the pyloric pacemaker kernel, and LP being the sole follower neuron that feeds back to the pacemakers (Hartline and Maynard, 1975; Bidaut, 1980; Selverston et al., 1982). The pyloric motor output is spontaneously active, making it amenable to detecting changes in premotor activity. Our results so far demonstrated that different sensory modalities are associated with distinct levels of premotor network activation and participation, but also that both premotor parameters were more similar between the VCN and bimodal conditions than between IV and the others (fig. 7D,E). We first monitored the changes in the motor pattern in the different sensory conditions. Following the trend of the premotor network, none of the motor pattern parameters measured (fig. 8D, firing frequency; fig. 8E, number of spikes per burst; fig. $8 \mathrm{~F}$, burst duration; fig. $8 \mathrm{G}$, intraburst firing frequency; fig. $8 \mathrm{H}$, cycle period) differed between the VCN and bimodal conditions. However, several parameters were significantly different between IV neuron stimulation and one or both of the other conditions, including PD and PY firing frequencies, PY and LP spikes per burst, PD and LP burst duration, PY intraburst firing frequencies, and the pyloric cycle period.

This indicates that premotor network activation and participation may play roles in driving changes in downstream motor output, but did not distinguish between the two. 
To specifically test whether the premotor network participation predicts changes in motor output, we first tested whether unique responses of individual neurons across sensory conditions (fig. 7E) influences motor output. By definition, a unique participant for one condition (e.g. neuron 1 is excited in IV condition) must share a similar response in the other two conditions (e.g. neuron 1 is inhibited by VCN and bimodal conditions). To correlate this with motor output, we calculated how the motor output differed between conditions, i.e. how unique the various motor pattern parameters were when compared between conditions (see Materials and Methods). For PD, we found a significant correlation between the premotor participation and its firing frequency (fig. 9A), but not for its burst duration (fig. 9B), spikes per burst (fig. 9C), and intraburst firing frequency (fig. 9D). No correlations were found between premotor participation and PY motor output (fig. 9F-I). For LP, firing frequency (fig. 9J) and intraburst firing frequency (fig. 9M) were not correlated. Burst duration (fig. 9K) and spikes per burst (fig. 9L), however, were both strongly correlated. Finally, the pyloric cycle period was correlated with premotor participation (fig. 9E). Thus, as similarities in premotor network participation increased, the motor patterns also became more similar (or less unique as plotted in fig. 9), indicating that the particular neurons involved and their distinct responses in the network response contributed to the motor output changes observed.

To then test whether network activation contributed to motor output, we correlated network activation in the different sensory conditions of each experiment with the corresponding changes in motor output, using the changes in motor output plotted in fig. 8. The firing frequencies and intraburst firing frequencies of the PD pacemakers (fig. 10A,D) were positively correlated with premotor network activation, while no significant 
relationships were found for other parameters of PD activity (fig. 10B,C). There was also a significant negative correlation of the pyloric cycle period with network activation (fig. $10 \mathrm{E})$, meaning that as network activation increased, the speed of pyloric rhythm increased along with it. PY was not strongly influenced by premotor network activation, as no significance was found for its burst duration (fig. 10G), spikes per burst (fig. 10H), or intraburst firing frequency (fig. 10I). However, we did find a significant positive correlation between premotor network activation and PY firing frequency (fig. 10F). LP activity was strongly correlated with premotor network activation: LP firing frequency (fig. 10J), burst duration (fig. 10K), and spikes per burst (fig. 10L) were all negatively correlated, while the sole non-significant measure of LP activity was its intraburst firing frequency (fig. 10M). Thus, CoG premotor network activation was an influential feature in the modulation of the downstream pyloric motor output, and both pacemaker and follower neuron activities are affected by it.

Network participation and activation converged partly onto the same motor pattern parameters: the four significant comparisons (figs. 9A,E,K,L) for network participation were also significant for network activation (figs. 10A,E,K,L). While network participation generated stronger correlations for how unique pyloric cycle period was $(R=-0.698$, fig. $9 E ; R=-0.589$, fig. $10 E)$, network activation was correlated with more of the motor output parameters than was network participation, and affected all three neurons of the pyloric circuit. Importantly, higher premotor network activation corresponded to increased firing frequencies in the PD pacemakers, and a correspondingly faster cycle period, indicating that premotor network activation determined motor pattern speed. 


\section{Discussion}

We investigated how different sensory conditions influence a premotor network that directly controls motor output (Diehl et al., 2013; Stein, 2017). We found that network activity was reflective of spatially distributed, highly overlapping, yet distinct network responses when comparing between IV and VCN sensory modalities. This feature was consistent with almost no variability across animals, despite the rather large anatomical variability observed in this region (Follmann et al., 2017). Our results also show a relationship between CoG premotor network activation and participation and pyloric activity. We conclude that a combinatorial code of premotor network activity is employed for sensory modality encoding, and that this coding scheme drives the various changes observed in the downstream pyloric motor circuit. Traditionally understood as a process associated with multiple higher nervous system structures, we show that multimodal information is locally differentiated via a premotor network, and this is directly translatable for the control of motor circuitry.

Potential mechanisms for the encoding of multimodal sensory information

Several hypotheses exist for the encoding of multimodal input, following the concepts developed in unisensory areas. (1) Rate coding may encode sensory stimuli from different sense organs in the firing frequencies of the involved neurons. This has been suggested for cortical regions: visual-tactile information is differentiated via spike train dynamics in primary visual and somatosensory cortices (Bieler et al., 2017), vestibularvisual information is involved in adaptive oculomotor responses (Nelson et al., 2017), and visual-proprioceptive discriminations are made in the posterior parietal cortex (VanGilder et al., 2016). Rate coding may also contribute to encoding of multimodal 
information in midbrain and brainstem: auditory and visual information yield disparate firing in the superior colliculus (Meredith et al., 1987; Miller et al., 2017), and neurons in the solitary nucleus are suggested to use rate coding or the specific timing of spikes relative to one another (often labeled temporal coding) for taste and odorant discrimination (Escanilla et al., 2015). A common argument for rate coding is that it may increase multimodal information capacity, i.e. rate coding allows encoding of a large set of parameters with fewer neurons while maintaining a broad and dynamic range of spike frequencies. This reinforces the validity of rate coding use in unisensory parameter distinction like odorant intensity (Sachse and Galizia, 2003), tastant type (Hallock and Di Lorenzo, 2006), and many mechanosensory and proprioceptive sense organs that convey feedback magnitude in response to movements (Birmingham et al., 1999; Proekt et al., 2007), but also may offer similar advantages for encoding of multimodal information. (2) Alternatively, sensory information can be encoded in the distinct activation of neurons, or their participation in the network (a combinatorial code). A combinatorial code may allow networks to make more robust distinctions when parameter space is limited (Osborne et al., 2008), making it less suitable for encoding wide ranges of inputs, but a prime candidate for distinguishing between different sensory modalities. Most evidence for combinatorial coding schemes comes from studies of unisensory stimulus encoding, such as odorant intensity, pheromone type, and object localization (Stengl et al., 1992; Sachse and Galizia, 2003; Palmer and Rosa, 2006), or from studies of multisensory encoding at the single-cell or coarse, large-scale network levels. In the basal ganglia, for example, distinct striatal subpopulations were described that either responded only to tactile stimuli, or to both 
tactile and visual inputs (Reig and Silberberg, 2014). While identifying individual unimodal and multimodal neurons has demonstrated both convergence and divergence of multisensory information in various brain areas, few studies have addressed how multisensory integration is achieved at the network level, and how these network responses drive behavioral output.

A combinatorial code of premotor network activation and participation determines motor output

Downstream premotor control networks such as the one studied here initiate and control motor output directly, and have long been known to process sensory information from different sense organs (Meredith and Stein, 1985; Burnett et al., 2004; Stein et al., 2006). In vertebrates, brainstem studies in lamprey, such as visual- and electrosensoryguided gaze reorientation (Kardamakis et al., 2016) and reticulospinal gating of steering (Kozlov et al., 2014), as well as similar findings in cat superior colliculus (Burnett et al., 2004), exemplify this feature. In invertebrates, there is direct evidence that combinatorial codes can be involved in decision making, and that network participation and activation change in different sensory and behavioral conditions. In leech, contributions of distinct but overlapping subsets of neurons are involved in the decision whether to swim or crawl (Briggman et al., 2005; Briggman and Kristan Jr., 2006), while in Aplysia and Tritonia, network dynamics change during the execution of a locomotor program and during behavioral sensitization (Bruno et al., 2015; Hill et al., 2015). In contrast to these studies, our approach allowed us to monitor premotor network activity and motor responses separately, because motor and premotor neurons reside in spatially distinct regions. Our work demonstrates how multimodal information is 
represented by a combinatorial code in the premotor network, and how different aspects of that code - network activation and network participation - modulate a downstream motor pattern.

In the stomatogastric nervous system, a small population of CoG projection neurons innervate the central pattern generators in the downstream stomatogastric ganglion, where they initiate and modulate distinct variants of gastric mill and pyloric motor patterns (Nusbaum et al., 2001; Stein, 2009; Blitz and Nusbaum, 2011) in different sensory conditions (Hedrich et al., 2009; Blitz and Nusbaum, 2012). It has been previously suggested that the combination of activated descending projection neurons may play a role in motor pattern selection (Stein, 2009). Conversely, variants of the gastric mill motor pattern appear to also be determined by changing the burst structure and firing patterns of the same neurons (Blitz and Nusbaum, 2008, 2012; Diehl et al., 2013). Our findings here address these two seemingly contradictory concepts and suggest that network activation and participation play a role in controlling downstream motor circuits. Network activation was correlated with seven of the thirteen parameters measured for pyloric motor output. The premotor CoG network affects the pyloric circuit via the modulatory properties of the descending projection neurons (Skiebe, 2001; Marder and Thirumalai, 2002; Nusbaum, 2013). In the sensory conditions we tested, different amounts of premotor neurons were excited and inhibited, leading to a change in the premotor network activation and its modulatory capacity. As modulatory capacity is changing, the downstream motor circuits receive different levels of modulation, resulting in changes of the pyloric motor output. Specifically, an increase in CoG network activation resulted in higher firing rates of the pyloric pacemakers, and shorter 
cycle periods of the pyloric rhythm. Simultaneously, burst duration and the number of spikes per burst of the LP follower neuron were reduced. Both parameters of LP activity have previously been shown to depend on cycle period, as a hallmark of phase maintenance of the pyloric motor pattern when cycle period changes (Soofi et al., 2012). Hence, most modulatory effects that resulted from increased modulatory capacity appeared to have been mediated preferentially via the pacemaker neurons. Our correlation analysis spans all sensory conditions, including the bimodal one.

Consequently, network activation was the main determinant of motor pattern speed in all conditions. This is consistent with our finding that $\mathrm{VCN}$ and bimodal stimulation caused similar CoG network activation and resulted in similar pyloric cycle periods, while network activation by the IV neurons was distinct and elicited distinct motor pattern speeds.

Our analyses also tracked how individual neurons changed their responses in the different sensory conditions ('participation'). CoG projection neuron firing patterns, although on a finer time scale than we report here, have been demonstrated to alter motor neuron and behavioral output. Our results generalize these findings to the population of premotor neurons: the more similar the population response was between conditions, the more similar the resultant motor patterns. This was the case despite the fact that less than $30 \%$ of the responding neurons showed differential responses in the tested conditions. Specifically, highly distinct premotor network responses resulted in distinct cycle periods of the pyloric rhythm, as well as in distinct LP burst durations and spike numbers. Changes in STG neuron burst durations have direct consequences for force production and contractions of the innervated muscles (Morris and Hooper, 1997), 
and motor pattern period directly determines the speed of the behavior - in this case the pace at which the pyloric filter apparatus runs (Stein, 2017). This implies that the uniqueness of the premotor neuron responses specifies the features of the behavioral output, which is consistent with previous findings that individual projection neurons can drive distinct behavioral patterns.

In summary, the CoG network responses to IV, VCN and bimodal conditions indicate that distinctions between sensory modalities are executed via the activation and participation of the premotor neurons. With only a few hundred neurons (Follmann et al., 2017), the CoG premotor network thus employs a combinatorial scheme to dynamically change CoG neuron responses in different sensory conditions, and control the downstream motor activity.

The bimodal condition is encoded in a new combination of premotor activities Theoretical and perceptual learning studies suggest that bimodal conditions yield new percepts that are not simply the sum of their unimodal components (Driver and Noesselt, 2008; Powers et al., 2012; McGovern et al., 2016). Part of a larger "binding problem", it is thought that if distinct sensory modalities are active within a particular temporal window, they may be perceptually bound as a single event. In terms of motor control, bimodal input can lead to enhanced or new behavioral responses that are distinct from the responses to the individual sensory modalities. Using visual and mechanosensory cues for guidance, fly odor tracking, for example, is significantly enhanced during flight, and bimodal processing is a prerequisite for this particular behavior (Duistermars and Frye, 2010). Similarly, localization of spatiotemporally 
concordant stimuli is achieved via combined auditory and visual input (Gingras et al., 2009).

Little is known about how different percepts and behavioral responses are generated, and what network responses underlie these processes. Most studies of multisensory processing, in particular those done in the context of motor control, consider one modality at a time. To encode a unique condition, network responses have to be distinct from the unimodal responses. Super- and sub-additive responses to bimodal input are quite common features in individual neurons, for example in integrating and non-integrating interneurons of the superior colliculus (Miller et al., 2017). This contrasts with some studies, such as moth tracking behavior (Roth et al., 2016), in which multimodal responses are the linear sum of two unimodal inputs. In the latter case, however, the bimodal condition serves the same behavioral function as the unimodal conditions. Either unimodal input alone can yield successful moth tracking behavior, so rather than being interpreted as its own percept, the bimodal condition acts to strengthen the robustness of the behavior through redundancy in sensory input. We found that the CoG premotor network response to the bimodal condition was distinct not only from the individual unimodal network responses, but also from the expected summation of both. In specific, more than half of the network activity was incongruent with the expected bimodal responses, demonstrating that the bimodal condition is not an additive function of unimodal input. While the identities of the premotor neurons recruited in each sensory condition studied here were different, it is important to note that the premotor response in the bimodal condition more resembled the mechanosensory condition that the chemosensory one, and that the pyloric rhythm also 
reflected these similarities. It is conceivable that, while encoded differentially in premotor activity, the mechanosensory and bimodal conditions are not functionally different within the immediate context of the behavior driven by the pyloric rhythm: when an animal encounters food, this will first activate the chemosensory IV neuron pathway, and IV activity will likely continue as long as food is present. Once food enters the stomach, the mechanosensory VCN pathway will activate in addition to the chemosensory pathway. This bimodal condition thus takes place without any unimodal mechanosensory activation. However, stomach distention can outlast the availability of food, for example when the meal is interrupted by a predator. This will lead to a loss of chemosensory activation, but maintains mechanosensory feedback. This sequence from unimodal chemosensory to bimodal and unimodal mechanosensory may allow the animal to differentiate an initial unfed and feeding state from a later fed but still foodprocessing state. For the pyloric filter apparatus, transitioning from the initial chemosensory condition to the bimodal condition is accompanied by changes in the motor drive as food entering the stomach seems an important trigger for digestion. Transitioning to the non-feeding state with a full stomach later on may not be quite as important since digestion continues. In contrast, perceiving all three sensory conditions as distinct as indicated by the unique CoG network responses, may be important for other behaviors, such as locomotion and decisions about flight or fight, which are mediated by the concerted actions of the brain and thoracic ganglia, both of which receive innervation from the CoG network (Kirby and Nusbaum, 2007; Follmann et al., 2017). 
Implications for multimodal encoding and motor pattern selection in higher order brain areas

To our knowledge, we are the first to demonstrate that behavioral plasticity in motor systems in response to distinct sensory modalities is mediated by a combinatorial code of activated neurons. Our results provide a mechanism by which more complex networks may encode bimodal sensory information, or more generally, converging neuronal information. This is reinforced by the more recent evidence for combinatorial coding in higher order brain regions, like the superior colliculus (Burnett et al., 2004; Kardamakis et al., 2016), basal ganglia (Reig and Silberberg, 2014), and even cortical areas (Osborne et al., 2008). The premotor network studied here is dedicated to processing of sensory information from several modalities, and determines behavioral output (Stein, 2009). Motor circuits such as central pattern generators and their control premotor circuits are quite distinct in function from neocortical circuits. Nevertheless, they also share several profound similarities, including spontaneous activity waves, rich dynamics and plasticity, powerful modulation, and engagement by sensory pathways (Yuste et al., 2005). Given that cortical circuits also share several anatomical and biophysical properties with downstream networks, such as repetition of small circuit modules, it is conceivable that the mechanisms present to distinguish and encode different sensory modalities foretell the basic principles of multimodal processing in cortical circuits, or even perception.

Information encoding forms the basis for how input from the external environment and internal feedback pathways yield appropriate behavioral responses. In general, how neuronal networks process multiple inputs simultaneously remains an enigmatic 
problem and is magnified as additional layers of processing are added. Our findings demonstrate that distinct neuronal populations encode multimodal information in a premotor region that controls behavioral responses, expanding the function of premotor neuronal circuits beyond motor control. The large repertoire of behavioral outputs associated with motor circuits makes combinatorial coding a viable option for representing a large assemblage of multimodal scenarios, and for information processing in general. Now more than ever should neuroscientists be capable of testing the importance of scale in understanding neural systems (namely, the human brain). The question of whether understanding a single neuron's activity can be useful in explaining a broad range of behavioral states seems to depend on the "what" and "where". Here it was essential to understand the single-neuron activities, but with respect to the larger network in which they were configured. Only through the combined network activities could we suggest that the premotor network operates on a level allowing it to modulate the pyloric rhythm, providing the appropriate modulatory drive to guide the pattern to a particular rhythmic state.

Author contributions: Christopher John Goldsmith (CJG) and Wolfgang Stein (WS) designed research; CJG and Rosangela Follmann (RF) performed research; CJG and RF analyzed data; CJG, RF, and WS wrote the paper. 


\section{REFERENCES}

Beenhakker MP, Blitz DM, Nusbaum MP. 2004. Long-lasting activation of rhythmic neuronal activity by a novel mechanosensory system in the crustacean stomatogastric nervous system. J Neurophysiol 91:78-91.

Beenhakker MP, Nusbaum MP. 2004. Mechanosensory activation of a motor circuit by coactivation of two projection neurons. J Neurosci 24:6741-50.

Benedek G, Eördegh G, Chadaide Z, Nagy A. 2004. Distributed population coding of multisensory spatial information in the associative cortex. Eur J Neurosci 20:525529.

Berkowitz A, Roberts A, Soffe SR. 2010. Roles for multifunctional and specialized spinal interneurons during motor pattern generation in tadpoles, zebrafish larvae, and turtles. Front Behav Neurosci 4:36.

Bidaut M. 1980. Pharmacological dissection of pyloric network of the lobster stomatogastric ganglion using picrotoxin. J Neurophysiol 44:1089-1101.

Bieler M, Sieben K, Cichon N, Schildt S, Röder B, Hanganu-Opatz IL. 2017. Rate and Temporal Coding Convey Multisensory Information in Primary Sensory Cortices. eNeuro 4.

Birmingham JT, Szuts ZB, Abbott LF, Marder E. 1999. Encoding of muscle movement on two time scales by a sensory neuron that switches between spiking and bursting modes. J Neurophysiol 82:2786-2797.

Blitz DM, Beenhakker MP, Nusbaum MP. 2004. Different sensory systems share projection neurons but elicit distinct motor patterns. J Neurosci 24:11381-90. 
Blitz DM, Nusbaum MP. 1997. Motor pattern selection via inhibition of parallel pathways. J Neurosci 17:4965-75.

Blitz DM, Nusbaum MP. 2008. State-dependent presynaptic inhibition regulates central pattern generator feedback to descending inputs. J Neurosci 28:9564-9574.

Blitz DM, Nusbaum MP. 2011. Neural circuit flexibility in a small sensorimotor system. Curr Opin Neurobiol 21:544-552.

Blitz DM, Nusbaum MP. 2012. Modulation of circuit feedback specifies motor circuit output. J Neurosci 32:9182-9193.

Böhm H, Dybek E, Heinzel HG. 2001. Anatomy and in vivo activity of neurons connecting the crustacean stomatogastric nervous system to the brain. J Comp Physiol A Sens Neural Behav Physiol 187:393-403.

Briggman KL, Abarbanel HD, Kristan Jr. WB. 2005. Optical imaging of neuronal populations during decision-making. Science (80- ) 307:896-901.

Briggman KL, Kristan WB. 2008. Multifunctional pattern-generating circuits. Annu Rev Neurosci 31:271-294.

Briggman KL, Kristan Jr. WB. 2006. Imaging dedicated and multifunctional neural circuits generating distinct behaviors. J Neurosci 26:10925-10933.

Bruno AM, Frost WN, Humphries MD. 2015. Modular deconstruction reveals the dynamical and physical building blocks of a locomotion motor program. Neuron 86:304-318.

Burnett LR, Stein BE, Chaponis D, Wallace MT. 2004. Superior colliculus lesions preferentially disrupt multisensory orientation. Neuroscience 124:535-547. 
Burrows M, Pflüger H-J. 1988. Positive feedback loops from proprioceptors involved in leg movements of the locust. J Comp Physiol A Sens Neural Behav Physiol 163:425-440.

Calabrese RL, De Schutter E. 1992. Motor-pattern-generating networks in invertebrates: modeling our way toward understanding. Trends Neurosci 15:439-445.

Cazalets JR, Nagy F, Moulins M. 1987. Suppressive control of a rhythmic central pattern generator by an identified modulatory neuron in crustacea. Neurosci Lett 81:267-272.

Christie AE, Stein W, Quinlan JE, Beenhakker MP, Marder E, Nusbaum MP. 2004. Actions of a histaminergic/peptidergic projection neuron on rhythmic motor patterns in the stomatogastric nervous system of the crab Cancer borealis. J Comp Neurol 469:153-169.

Clemens S, Massabuau JC, Legeay A, Meyrand P, Simmers J. 1998a. In vivo modulation of interacting central pattern generators in lobster stomatogastric ganglion: influence of feeding and partial pressure of oxygen. J Neurosci 18:27882799.

Clemens S, Meyrand P, Simmers J. 1998b. Feeding-induced changes in temporal patterning of muscle activity in the lobster stomatogastric system. Neurosci Lett 254:65-68.

Daghfous G, Green WW, Alford ST, Zielinski BS, Dubuc R. 2016. Sensory Activation of Command Cells for Locomotion and Modulatory Mechanisms: Lessons from Lampreys. Front Neural Circuits 10:1-17. 
Diehl F, White RS, Stein W, Nusbaum MP. 2013. Motor circuit-specific burst patterns drive different muscle and behavior patterns. J Neurosci 33:12013-12029.

Driver J, Noesselt T. 2008. Multisensory interplay reveals crossmodal influences on "sensory-specific" brain regions, neural responses, and judgments. Neuron 57:1123.

Duistermars BJ, Frye MA. 2010. Multisensory integration for odor tracking by flying Drosophila. Behavior, circuits and speculation. Commun Integr Biol 3:1-5.

Ehmer B, Gronenberg W. 2002. Segregation of visual input to the mushroom bodies in the honeybee (Apis mellifera). J Comp Neurol 451:362-373.

Escanilla OD, Victor JD, Di Lorenzo PM. 2015. Odor-taste convergence in the nucleus of the solitary tract of the awake freely licking rat. J Neurosci 35:6284-97.

Follmann R, Goldsmith CJ, Stein W. 2017. Spatial distribution of intermingling pools of projection neurons with distinct targets: A 3D analysis of the commissural ganglia in Cancer borealis. J Comp Neurol 525:1827-1843.

Fu K-MG, Johnston TA, Shah AS, Arnold L, Smiley J, Hackett TA, Garraghty PE, Schroeder CE. 2003. Auditory cortical neurons respond to somatosensory stimulation. J Neurosci 23:7510-7515.

Getting PA, Dekin MS. 1985. Mechanisms of pattern generation underlying swimming in Tritonia. IV. Gating of central pattern generator. J Neurophysiol 53:466-480.

Ghazanfar AA, Schroeder CE. 2006. Is neocortex essentially multisensory? Trends Cogn Sci 10:278-285.

Gingras G, Rowland B a, Stein BE. 2009. The Differing Impact of Multisensory and Unisensory Integration on Behavior. J Neurosci 29:4897-4902. 
Goldsmith CJ, Stadele C, Stein W. 2014. Optical imaging of neuronal activity and visualization of fine neural structures in non-desheathed nervous systems. PLoS One 9:e103459.

Hackett TA, Schroeder CE. 2009. Multisensory integration in auditory and auditoryrelated areas of cortex. Hear Res 258:1-3.

Hallock RM, Di Lorenzo PM. 2006. Temporal coding in the gustatory system. Neurosci Biobehav Rev 30:1145-1160.

Harris-Warrick RM, Johnson BR. 2010. Checks and balances in neuromodulation. Front Behav Neurosci 4:1-9.

Hartline DK, Maynard DM. 1975. Motor patterns in the stomatogastric ganglion of the lobster Panulirus argus. J Exp Biol 62:405-420.

Hedrich UB, Smarandache CR, Stein W. 2009. Differential activation of projection neurons by two sensory pathways contributes to motor pattern selection. $\mathrm{J}$ Neurophysiol 102:2866-2879.

Hedrich UBS, Stein W. 2008. Characterization of a descending pathway: activation and effects on motor patterns in the brachyuran crustacean stomatogastric nervous system. J Exp Biol 211:2624-37.

Hill ES, Vasireddi SK, Wang J, Bruno AM, Frost WN. 2015. Memory Formation in Tritonia via Recruitment of Variably Committed Neurons. Curr Biol 25:2879-2888. Juvin L, Grätsch S, Trillaud-Doppia E, Gariépy J-F, Büschges A, Dubuc R. 2016. A Specific Population of Reticulospinal Neurons Controls the Termination of Locomotion. Cell Rep 15:2377-2386. 
Kardamakis AA, Pérez-Fernández J, Grillner S. 2016. Spatiotemporal interplay between multisensory excitation and recruited inhibition in the lamprey optic tectum. Elife 5.

Katz PS. 2016. Evolution of central pattern generators and rhythmic behaviours. Philos Trans R Soc Lond B Biol Sci 371:20150057.

Kiehn O. 2006. Locomotor circuits in the mammalian spinal cord. Annu Rev Neurosci 29:279-306.

Kirby MS, Nusbaum MP. 2007. Central nervous system projections to and from the commissural ganglion of the crab Cancer borealis. Cell Tissue Res 328:625-637.

Koulakov A, Gelperin A, Rinberg D. 2007. Olfactory Coding With All-or-Nothing Glomeruli. J Neurophysiol 98:3134-3142.

Kozlov A, Huss M, Lansner A, Kotaleski JH, Grillner S. 2009. Simple cellular and network control principles govern complex patterns of motor behavior. Proc Natl Acad Sci 106:20027-20032.

Kozlov AK, Kardamakis A a, Hellgren Kotaleski J, Grillner S. 2014. Gating of steering signals through phasic modulation of reticulospinal neurons during locomotion. Proc Natl Acad Sci 111:3591-3596.

Marder E, Thirumalai V. 2002. Cellular, synaptic and network effects of neuromodulation. Neural Networks 15:479-493.

McGovern DP, Roudaia E, Newell FN, Roach NW. 2016. Perceptual learning shapes multisensory causal inference via two distinct mechanisms. Sci Rep 6:24673. Meijer GT, Montijn JS, Pennartz CMA, Lansink CS. 2017. Audio-visual modulation in mouse $\mathrm{V} 1$ depends on cross-modal stimulus configuration and congruency. $\mathrm{J}$ Neurosci 37:468-17. 
Meredith MA, Nemitz JW, Stein BE. 1987. Determinants of multisensory integration in superior colliculus neurons. I. Temporal factors. J Neurosci 7:3215-3229.

Meredith MA, Stein BE. 1985. Descending efferents from the superior colliculus relay integrated multisensory information. Science 227:657-9.

Miller RL, Stein BE, Rowland BA. 2017. Multisensory integration uses a real time unisensory-multisensory transform. J Neurosci 37:2767-16.

Morgan PT, Jing J, Vilim FS, Weiss KR. 2002. Interneuronal and peptidergic control of motor pattern switching in Aplysia. J Neurophysiol 87:49-61.

Morris LG, Hooper SL. 1997. Muscle response to changing neuronal input in the lobster (Panulirus interruptus) stomatogastric system: slow muscle properties can transform rhythmic input into tonic output. J Neurosci 17:3433-42.

Nelson AB, Faulstich M, Moghadam S, Onori K, Meredith A, du Lac S. 2017. BK Channels Are Required for Multisensory Plasticity in the Oculomotor System. Neuron 93:211-220.

Noel J-P, Wallace MT, Orchard-Mills E, Alais D, Van der Burg E. 2015. True and Perceived Synchrony are Preferentially Associated With Particular Sensory Pairings. Sci Rep 5:17467.

Norris BJ, Coleman MJ, Nusbaum MP. 1996. Pyloric motor pattern modification by a newly identified projection neuron in the crab stomatogastric nervous system. J Neurophysiol 75:97-108.

Nusbaum MP. 2013. Modulatory Projection Neurons. Encycl Neurosci :http://www.springerreference.com/docs/html/chapter. 
Nusbaum MP, Beenhakker MP. 2002. A small-systems approach to motor pattern generation. Nature 417:343-350.

Nusbaum MP, Blitz DM. 2012. Neuropeptide modulation of microcircuits. Curr Opin Neurobiol 22:592-601.

Nusbaum MP, Blitz DM, Swensen AM, Wood D, Marder E. 2001. The roles of cotransmission in neural network modulation. Trends Neurosci 24:146-154.

Nusbaum MP, Weimann JM, Golowasch J, Marder E. 1992. Presynaptic control of modulatory fibers by their neural network targets. J Neurosci 12:2706-2714.

Obaid AL, Loew LM, Wuskell JP, Salzberg BM. 2004. Novel naphthylstyryl-pyridium potentiometric dyes offer advantages for neural network analysis. J Neurosci Methods 134:179-190.

Osborne LC, Palmer SE, Lisberger SG, Bialek W. 2008. The neural basis for combinatorial coding in a cortical population response. J Neurosci 28:13522-31.

Palmer SM, Rosa MG. 2006. A distinct anatomical network of cortical areas for analysis of motion in far peripheral vision. Eur J Neurosci 24:2389-2405.

Pennartz CMA. 2009. Identification and integration of sensory modalities: Neural basis and relation to consciousness. Conscious Cogn 18:718-739.

Petersen CC, Sakmann B. 2001. Functionally independent columns of rat somatosensory barrel cortex revealed with voltage-sensitive dye imaging. $\mathrm{J}$ Neurosci 21:8435-8446.

Powers AR, Hevey MA, Wallace MT. 2012. Neural correlates of multisensory perceptual learning. J Neurosci 32:6263-74. 
Proekt A, Jing J, Weiss KR. 2007. Multiple contributions of an input-representing neuron to the dynamics of the aplysia feeding network. J Neurophysiol 97:3046-3056.

Quiroga RQ, Nadasdy Z, Ben-Shaul Y. 2004. Unsupervised Spike Detection and Sorting with Wavelets and Superparamagnetic Clustering. Neural Comput $16: 1661-1687$.

Rauschecker JP, Tian B, Hauser M. 1995. Processing of complex sounds in nonprimary auditory cortex of the rhesus monkey. Science (80- ) 268:111-114.

Le Ray D, Juvin L, Ryczko D, Dubuc R. 2011. Supraspinal control of locomotion. The mesencephalic locomotor region. Prog Brain Res 188:51-70.

Reig R, Silberberg G. 2014. Multisensory Integration in the Mouse Striatum. Neuron 83:1200-1212.

Rohe T, Noppeney U. 2016. Distinct Computational Principles Govern Multisensory Integration in Primary Sensory and Association Cortices. Curr Biol 26:509-514.

Roth E, Hall RW, Daniel TL, Sponberg S. 2016. Integration of parallel mechanosensory and visual pathways resolved through sensory conflict. Proc Natl Acad Sci 113:12832-12837.

Sachse S, Galizia CG. 2003. The coding of odour-intensity in the honeybee antennal lobe: local computation optimizes odour representation. Eur J Neurosci 18:2119_ 2132.

Schnell B, Ros IG, Dickinson MH. 2017. A Descending Neuron Correlated with the Rapid Steering Maneuvers of Flying Drosophila. Curr Biol 27:1200-1205.

Schroeder CE, Foxe J. 2005. Multisensory contributions to low-level, "unisensory" processing. Curr Opin Neurobiol 15:454-458. 
Selverston Al. 2010. Invertebrate central pattern generator circuits. Philos Trans R Soc Lond B Biol Sci 365:2329-2345.

Selverston AI, Miller JP, WadepuhI M. 1982. Local circuits for the generation of rhythmic motor patterns. J Physiol 78:748-754.

Sen R, Wu M, Branson K, Robie A, Rubin GM, Dickson BJ. 2017. Moonwalker Descending Neurons Mediate Visually Evoked Retreat in Drosophila. Curr Biol $27: 766-771$.

Sigvardt KA, Mulloney B. 1982. Sensory alteration of motor patterns in the stomatogastric nervous system of the spiny lobster Panulirus interruptus. J Exp Biol 97:137-152.

Skiebe P. 2001. Neuropeptides are ubiquitous chemical mediators: Using the stomatogastric nervous system as a model system. J Exp Biol 204:2035-2048.

Soofi W, Archila S, Prinz AA. 2012. Co-variation of ionic conductances supports phase maintenance in stomatogastric neurons. J Comput Neurosci 33:77-95.

Stein W. 2009. Modulation of stomatogastric rhythms. J Comp Physiol A Neuroethol Sens Neural Behav Physiol 195:989-1009.

Stein W. 2017. Stomatogastric Nervous System. Comp Insect Physiol Biochem Pharmacol 1:371-406.

Stein W, Buschges A, Bassler U. 2006. Intersegmental transfer of sensory signals in the stick insect leg muscle control system. J Neurobiol 66:1253-1269.

Stein W, Schmitz J. 1999. Multimodal convergence of presynaptic afferent inhibition in insect proprioceptors. J Neurophysiol 82:512-514. 
Stengl M, Zufall F, Hatt H, Hildebrand JG. 1992. Olfactory receptor neurons from antennae of developing male Manduca sexta respond to components of the species-specific sex pheromone in vitro. J Neurosci 12:2523-31.

VanGilder P, Shi Y, Apker G, Dyson K, Buneo CA. 2016. Multisensory Interactions Influence Neuronal Spike Train Dynamics in the Posterior Parietal Cortex. PLoS One 11:e0166786.

Venezia JH, Vaden KI, Rong F, Maddox D, Saberi K, Hickok G. 2017. Auditory, Visual and Audiovisual Speech Processing Streams in Superior Temporal Sulcus. Front Hum Neurosci 11:174.

Wang X, Lu T, Bendor D, Bartlett E. 2008. Neural coding of temporal information in auditory thalamus and cortex. Neuroscience 154:294-303.

Wiersma CA. 1957. On the number of nerve cells in a crustacean central nervous system. Acta Physiol Pharmacol Neerl 6:135-142.

Xin Y, Weiss KR, Kupfermann I. 1996. An identified interneuron contributes to aspects of six different behaviors in Aplysia. J Neurosci 16:5266-79.

Yeh S-Y, Huang W, Wang W, Ward CS, Chao ES, Wu Z, Tang B, Tang J, Sun JJ, Esther van der Heijden M, Gray PA, Xue M, Ray RS, Ren D, Zoghbi HY. 2017. Respiratory Network Stability and Modulatory Response to Substance P Require Nalcn. Neuron 94:294-303.e4.

Yuste R, MacLean JN, Smith J, Lansner A. 2005. The cortex as a central pattern generator. Nat Rev Neurosci 6:477-483. 


\section{FIGURES}

A

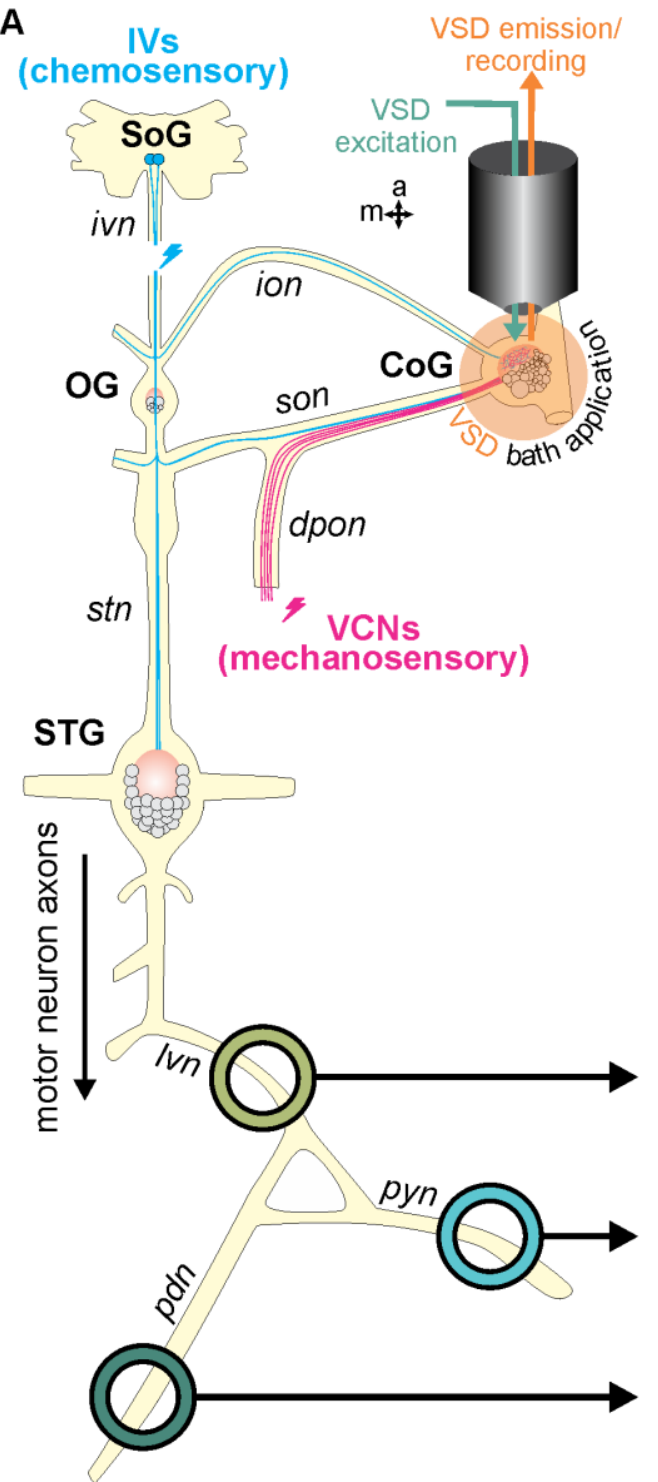

B
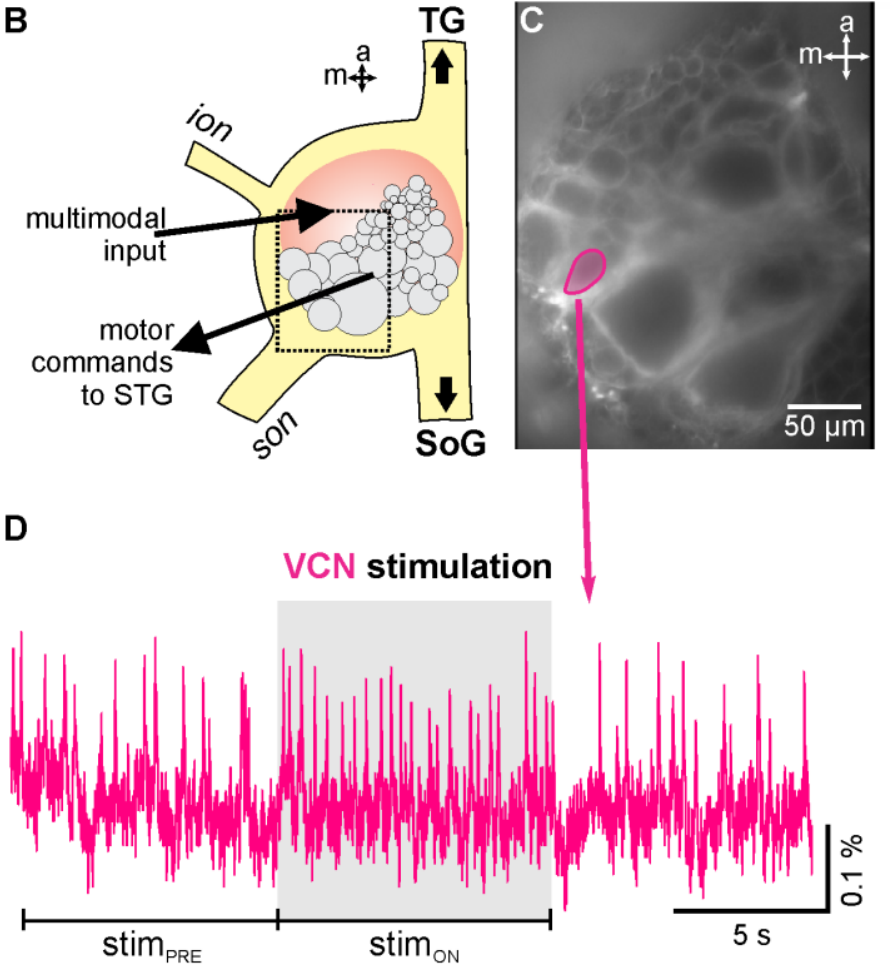

E
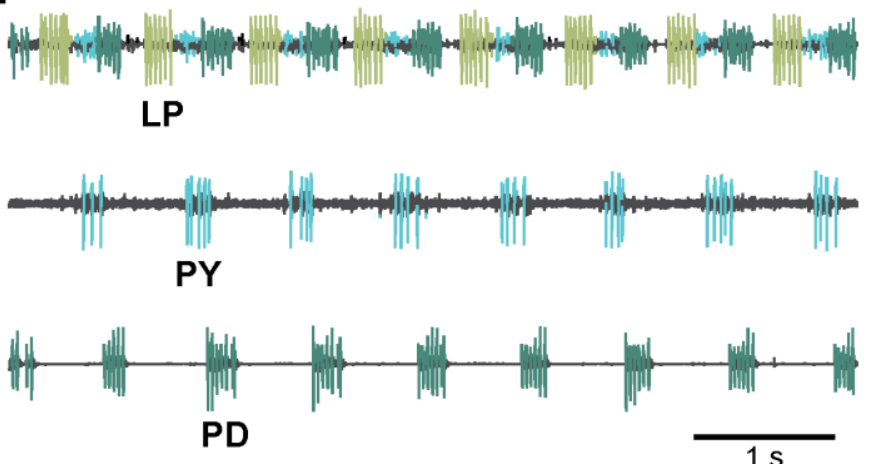

Fig. 1. Voltage-sensitive dye imaging in a multifunctional motor system. (A) The STNS consists of four ganglia: the stomatogastric ganglion (STG), the esophageal ganglion $(\mathrm{OG})$, and the bilaterally paired CoGs (only right CoG shown here). The chemosensory IV neurons (cyan) descend from the supraesophageal ganglion (SoG, 'brain') and innervate the CoGs via the ivn, ion and son, and the STG via the stn. The mechanosensory VCNs (magenta) innervate the CoGs via the dpon. Voltage-sensitive dye (VSD) imaging was used to monitor CoG premotor neuronal activity. ion, inferior esophageal nerve; dpon, dorsal posterior esophageal nerve; ivn, inferior ventricular nerve; son, superior esophageal nerve; stn, stomatogastric nerve. (B) While CoG neurons project to many different locations throughout the nervous system such as the thoracic ganglion (TG) and the brain (SoG), a subset of the premotor neurons localized 
in the medial-posterior area of the ganglion (dotted box) project to downstream motor circuits (arrow) in the STG. (C) Example image of neuronal cell bodies in the premotor area that contains descending projection neurons that control the STG motor circuits. About 80 distinct cell bodies are distinguishable via their brightly fluorescing cell boundaries. All neurons in a single focal plane were imaged simultaneously. (D) Optical trace from a single neuron in (C), showing the change in spike activity in response to VCN stimulation. (E) Sample traces of the downstream pyloric motor neurons recorded extracellularly from motor nerves posterior to the STG. Action potential (spike) information was attained for the lateral pyloric (LP) neuron, the pyloric constrictor (PY) neurons, and the pyloric dilator (PD) neurons via recordings of the lateral ventricular nerve $(I v n)$, the pyloric constrictor nerve $(p y n)$, and the pyloric dilator nerve $(p d n)$, respectively. 

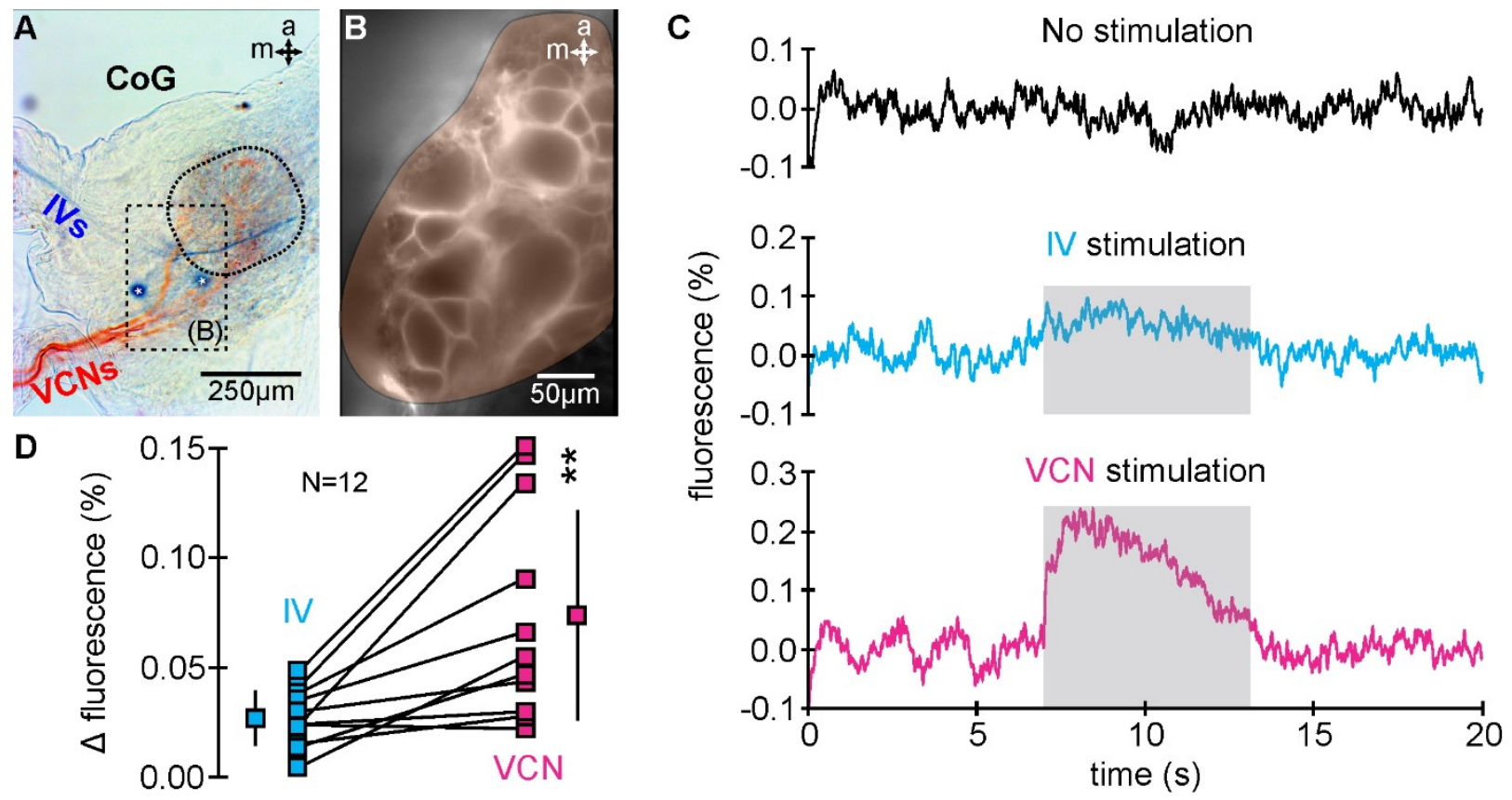

Fig. 2. Multiple sensory modalities converge onto the commissural ganglion (CoG) premotor region and elicit distinct neuronal responses. (A) Image of backfilled axons showing the convergence of axonal projections from the inferior ventricular neurons (IVs, blue) and the ventral cardiac neurons (VCNs, orange) in the CoG neuropil (dotted area). Dashed rectangle indicates the premotor region of interest. Asterisks indicate cell bodies stained by backfills of the inferior ventricular nerve (ivn) backfill, representing neurons that project from the CoGs to the brain (6 axons). No CoG cell bodies were stained with VCN (dorsal posterior esophageal nerve, dpon) backfills, confirming that all axons stained in the dpon project towards the CoG. Orientation: "a", anterior; "m", medial. (B) Fluorescence image of the CoG premotor region that contains the cell bodies of the neurons involved in control of downstream stomatogastric motor circuits. Transparent overlay indicates the spatially averaged area used for whole-ganglion fluorescence measurements. (C) Averaged fluorescence signals from all neurons without stimulation (top), during IV stimulation (middle), and during VCN stimulation (bottom). IV and VCN stimulation both caused an increase in fluorescence. Stimulus trains lasted for 6 seconds (shaded region). (D) Plot of the average changes in fluorescence between pre-stimulation control and stimulation ( $\Delta$ fluorescence) for both stimulus conditions. The magnitude of the CoG response was significantly larger during VCN stimulation ( $\mathrm{N}=12,{ }^{* *} \mathrm{p}<0.01$, paired $\mathrm{t}$-test). Data from individual ganglia and their mean $\pm \mathrm{SD}$ are provided. 


\section{VSD Imaging reliably detects CoG neuron firing}

A

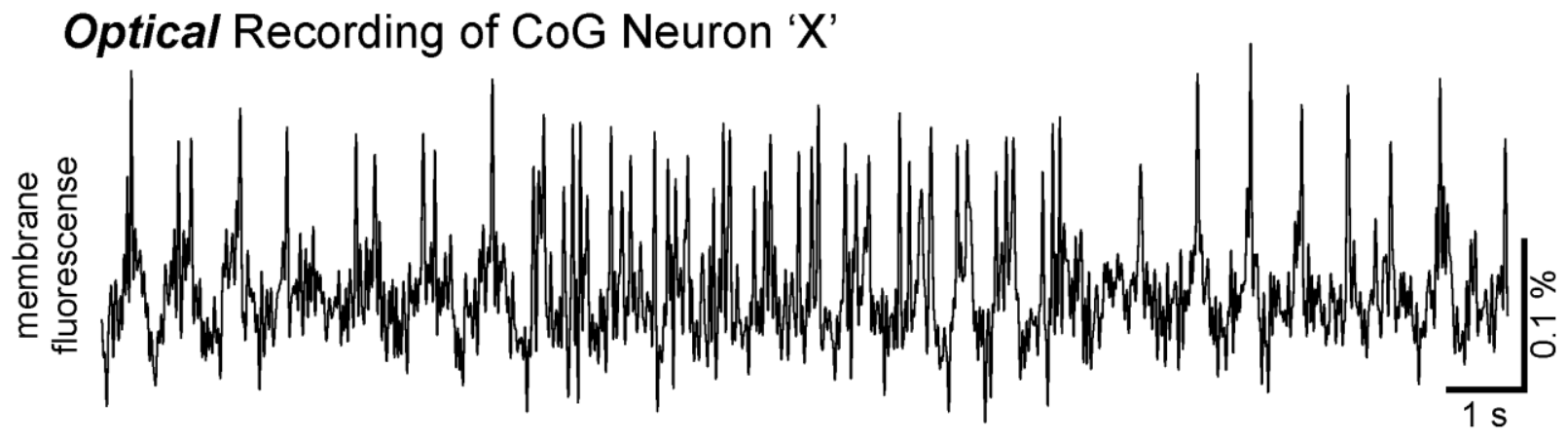

Intracellular Current Clamp Recording of CoG Neuron ' $\mathrm{X}$ '

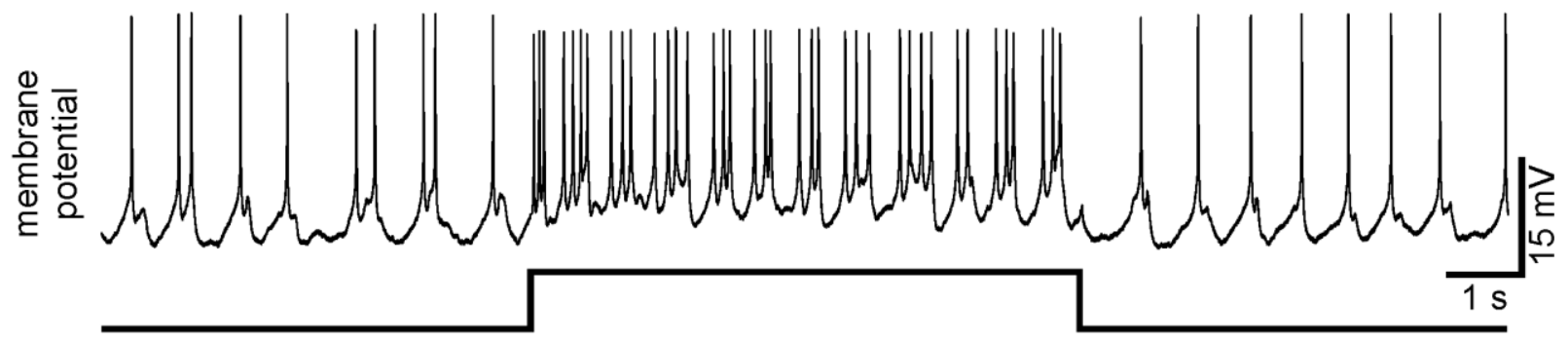

intracellular current injection $(1 \mathrm{nA})$

B
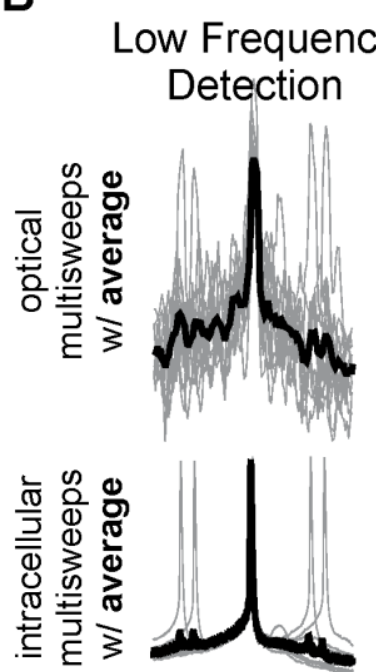

C

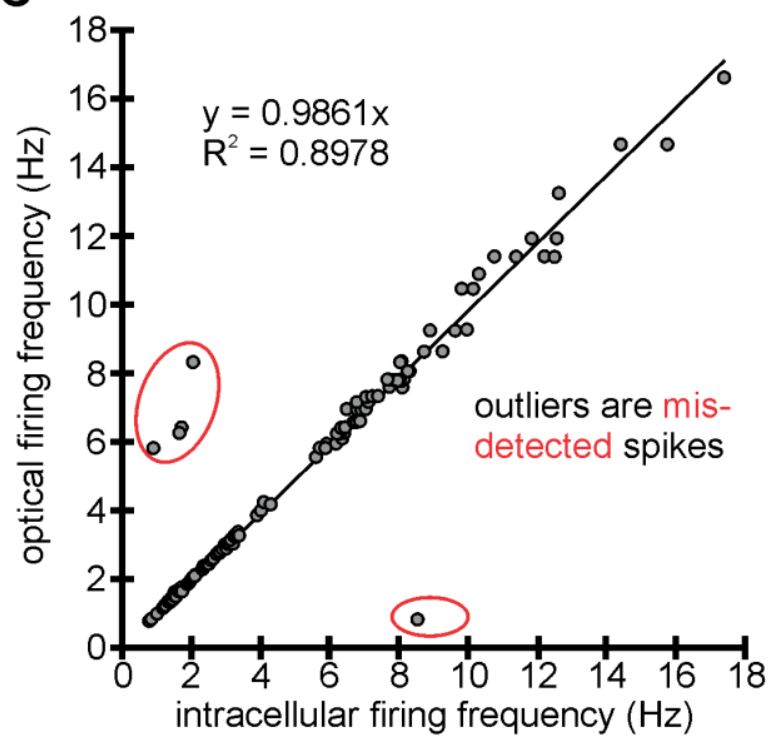

Fig. 3. Comparison of optical recording with voltage-sensitive dyes with an intracellular recording acquired simultaneously in the same CoG neuron ' $X$ '. (A) Single-sweep measurements from a representative optical (TOP) and intracellular (BOTTOM) recording. This example demonstrates accuracy of optical spike detection during both lower $(\sim 1 \mathrm{~Hz})$ and higher $(15-20 \mathrm{~Hz})$ firing frequencies with a depolarizing current 
injection. (B) Optical (TOP) and intracellular (BOTTOM) multisweeps (gray overlaid traces) for low and high spike frequencies. Waveform averages (black thick trace) are overlain on the multisweep traces. (C) Using spike-to-spike latency measurements, we compared the efficiency of the optical recording in detecting spikes. Mis-detected spikes are outliers on the scatter plot (circled in red), and are indicative of either over-detection or under-detection of spikes in the optical recording. Only 6 out of 114 spikes were misdetected in this analysis (95\% accuracy). 
A

(1)

(2)

(3)

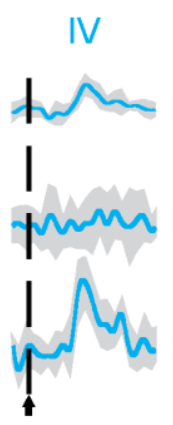

VCN

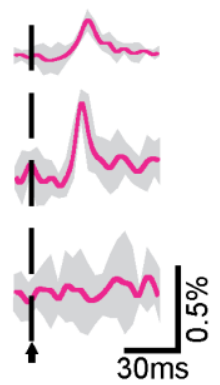

D

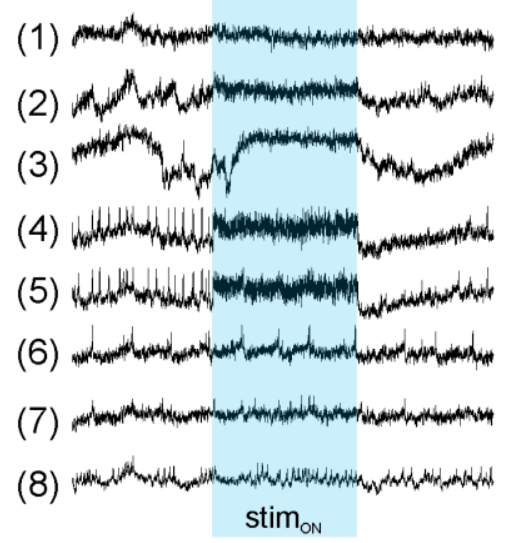

B
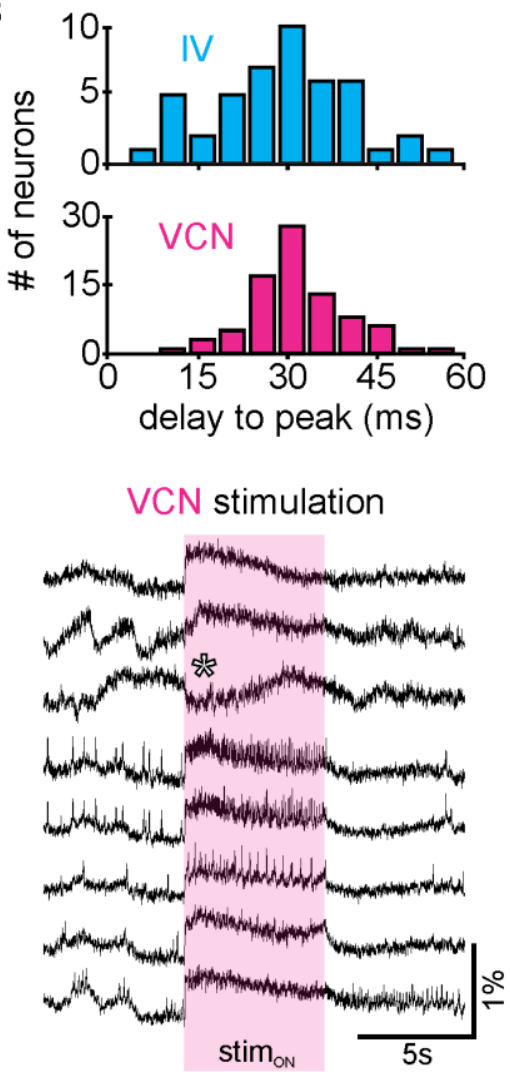

C

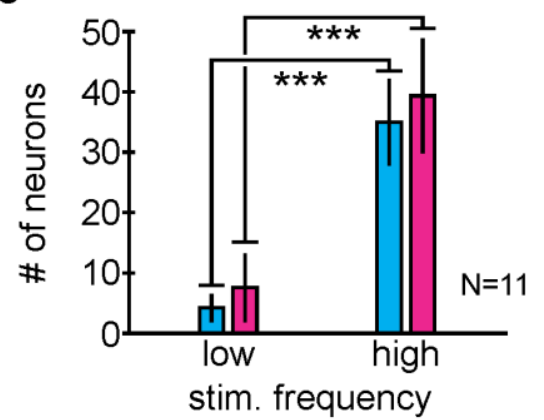

E

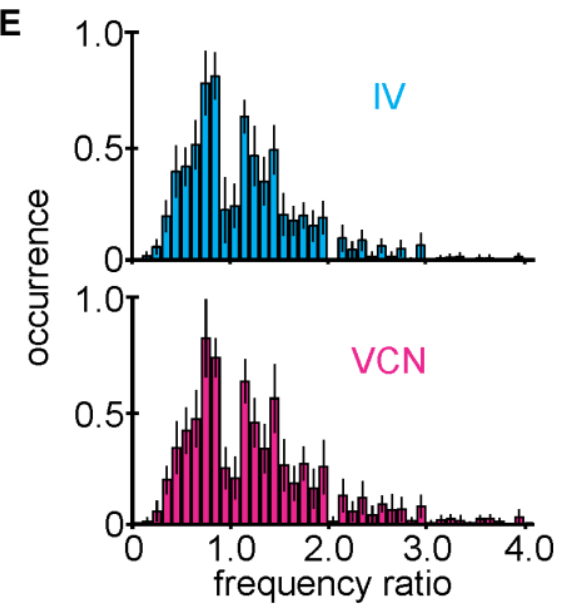

Fig. 4. Neuronal response latencies and spike frequencies are similar between sensory modalities. (A) Responses of three individual neurons (1-3) to IV (left) and VCN (right) to low frequency stimuli $(1 \mathrm{~Hz})$ in the same preparation. Vertical dashed lines indicate stimulus onset. Data are averaged traces of 6 trials \pm SD (shaded region). (B) Distribution of response latencies (time from stimulus onset to peak neuronal response) for neurons excited by IV (top) and VCN (bottom) low frequency stimuli $(1 \mathrm{~Hz})$. There were no significant differences between distributions ( $N=11$ ganglia, $n=129$ neurons, $\mathrm{p}=0.140$, two-sample Kolmogorov-Smirnov test). Mean delays, IV: $29.7 \pm 11.4 \mathrm{~ms}, \mathrm{VCN}$ : 31.9 $\pm 7.7 \mathrm{~ms}$. (C) Excitatory responses to low $(1 \mathrm{~Hz}$ ) and high (IV: $40 \mathrm{~Hz} ; \mathrm{VCN}: 15 \mathrm{~Hz}$ ) frequency stimuli. High frequencies excited more neurons in both sensory conditions. (D) High frequency sensory stimuli yielded various neuronal responses that altered spontaneous activity levels. Sample activity traces from individual neurons during IV stimulation (left) and VCN stimulation (right) in the same preparation. Shaded regions indicate the period when sensory stimulation was applied. (E) Spike frequency ratio distributions of all neurons for IV (left) and VCN (right) inputs. No differences were found ( $N=12$ ganglia, $n=878$ neurons, $p=0.144$, two-sample Kolmogorov-Smirnov test). Data are mean \pm SD. 

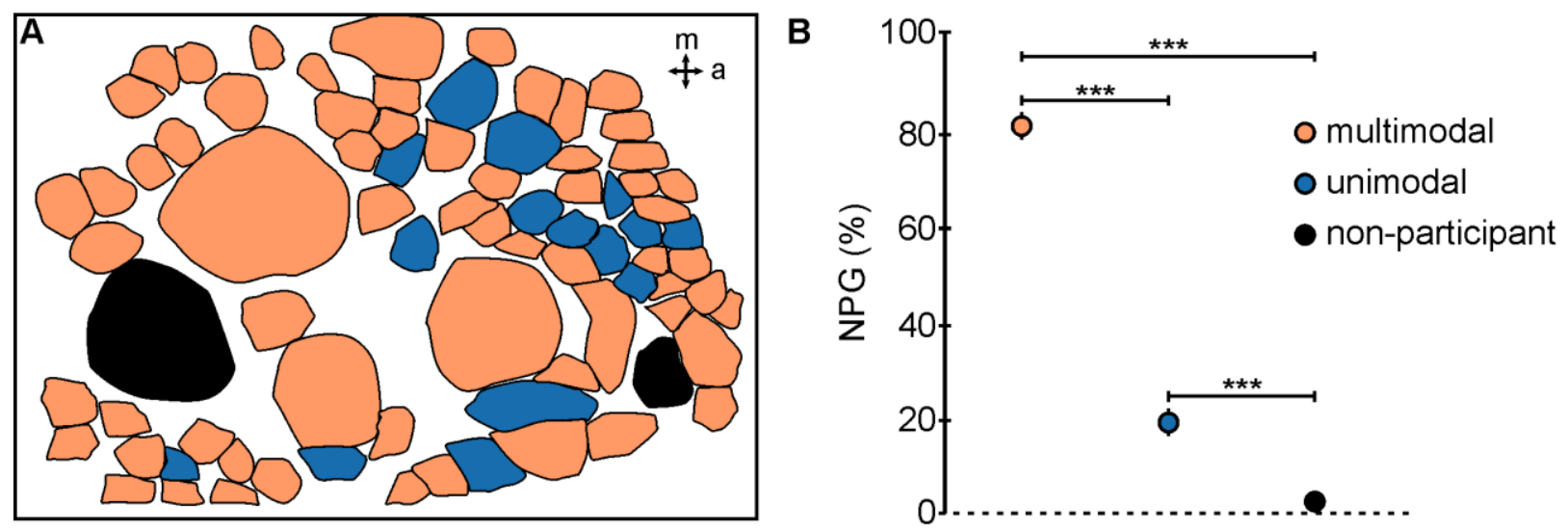

C IV stimulation
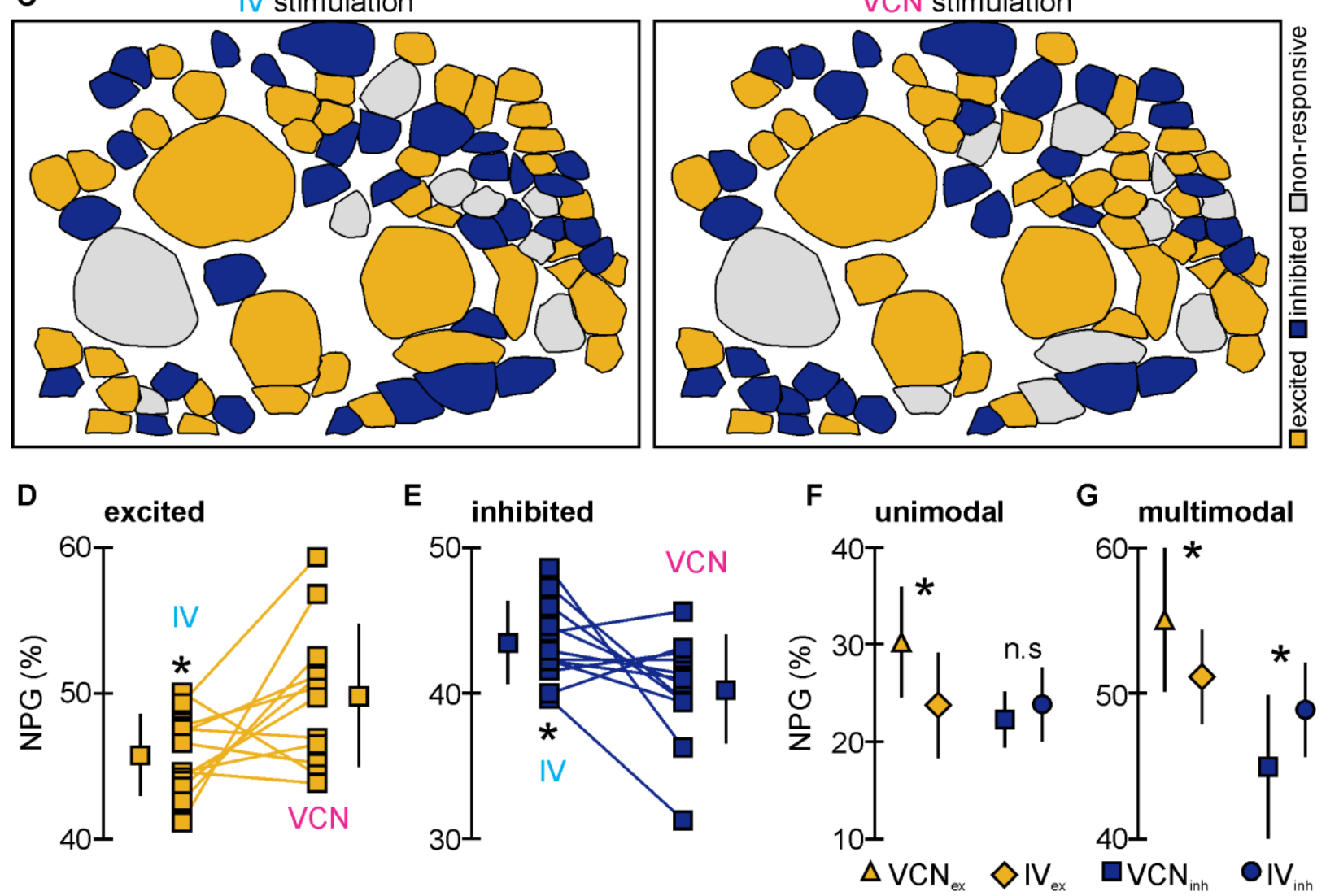

Fig. 5. Network excitation and inhibition is modality-specific. (A) Example map showing multimodal (neurons that responded to both sensory modalities, orange cell bodies), unimodal (neurons that responded to only one sensory modality, blue cell bodies) and non-participant (neurons that did not respond to either modality, black cell bodies) CoG neurons. (B) Across ganglia, a significantly greater proportion of multimodal neurons was found in comparison to the proportion unimodal neurons, and only a small fraction of neurons were non-participants. The plot shows the normalized number of neurons per ganglion (NPG). Data are mean $\pm \mathrm{SD},\left({ }^{* * *} p<0.001\right.$, repeated measures ANOVA with Tukey-Kramer post-hoc test). (C) Example response maps illustrating the types of 
neuronal responses for induced by stimulation of IV (left) and VCN (right) pathways: excited (yellow), inhibited (blue), or non-responsive (gray). (D) Proportion of neurons per ganglion that were excited by IV and VCN stimulation, as indicated. VCN stimulation excited a significantly larger proportion of CoG neurons than IV stimulation. (E) Proportion of neurons per ganglion that were inhibited by IV and VCN stimulation, as indicated. IV stimulation inhibited a significantly larger number of CoG neurons than VCN stimulation. (F) Normalized number of neurons per ganglion of unimodal response types. The largest proportion of unimodal neurons were excited by VCN stimulation. (G) Normalized number of neurons per ganglion of multimodal response types. Mirroring the results in Figure III--5D and Figure III-5E, a larger proportion of (in this case multimodal) CoG neurons were excited by VCN stimulation, and a larger proportion were inhibited by IV stimulation. Data $(D, E, F$ and $G)$ are mean $\pm S D(N=12$ ganglia for each response type compared, n.s.: not significant, ${ }^{*} p<0.05$, paired t-test). 
VCN stimulation

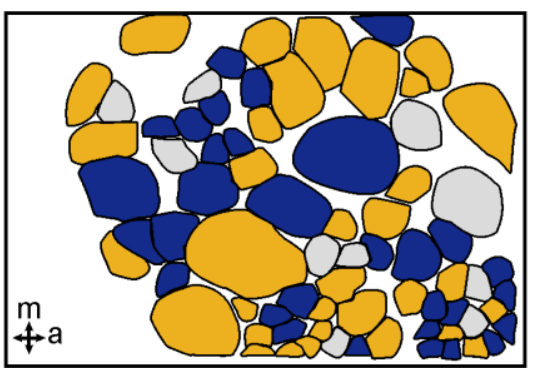

COP

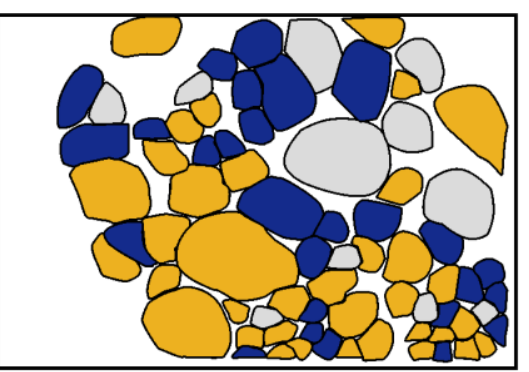

bimodal stimulation

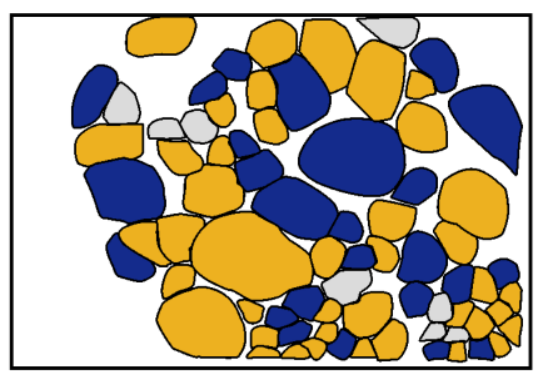

B
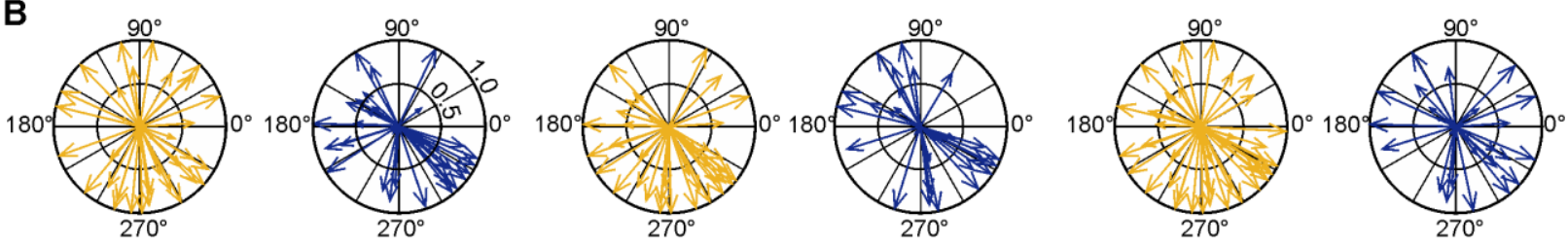

C

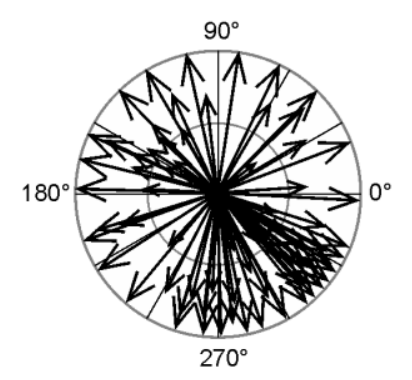

D

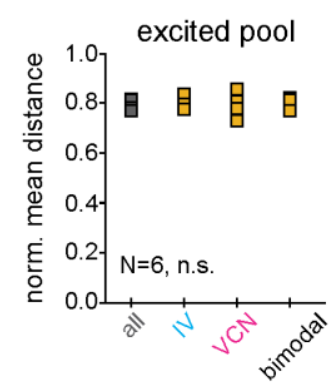

neuronal spread from origin inhibited pool
E

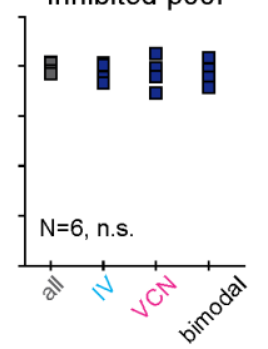

neuronal angular distribution

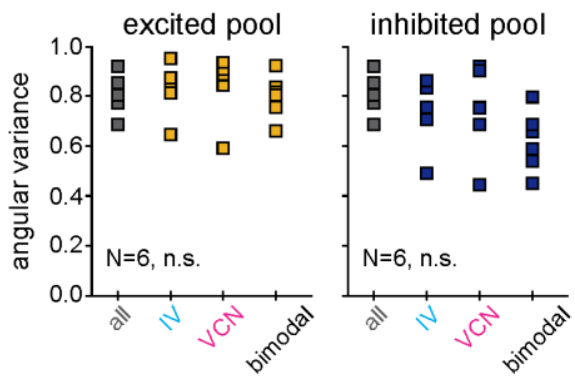

Fig. 6. No differences in the spatial distributions of premotor CoG neurons were found between different sensory conditions or neuronal response types. (A) Maps of excited (yellow), inhibited (blue), and non-responsive (gray) neurons according to their responses to (from left to right) IV stimulation, VCN stimulation, and bimodal stimulation. (B) Vector plot of excited and inhibited neurons' locations for (from left to right) IV, VCN, and bimodal stimulation with respect to the center of the imaged premotor (see Materials and Methods). Vector lengths correspond to the normalized distance from the center. Same example as in (A). (C) Vector plot of all imaged cell bodies from the example used in (A). (D) Normalized mean distance from the origin for each condition $(\mathrm{N}=6$ ganglia, $\mathrm{p}=0.24$ for excited neurons, $\mathrm{p}=0.87$ for inhibited neurons, repeated measures ANOVA, Tukey-Kramer post-hoc test). (E) Angular variance for each sensory condition ( $N=6$ ganglia, $p=0.88$ for excited neurons, $p=0.11$ for inhibited neurons, repeated measures ANOVA, Tukey-Kramer post-hoc test). No differences in distance and angular distributions were found across sensory condition and response types. 
A

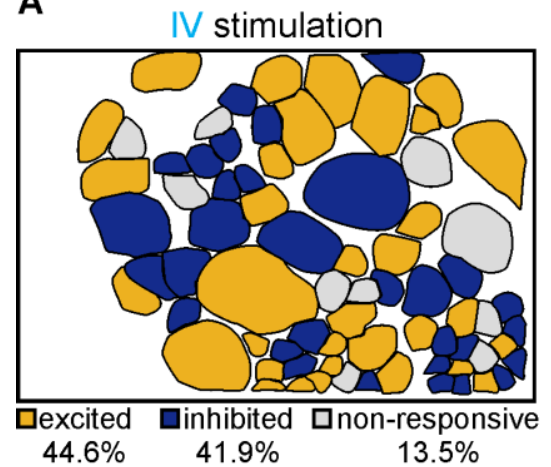

B

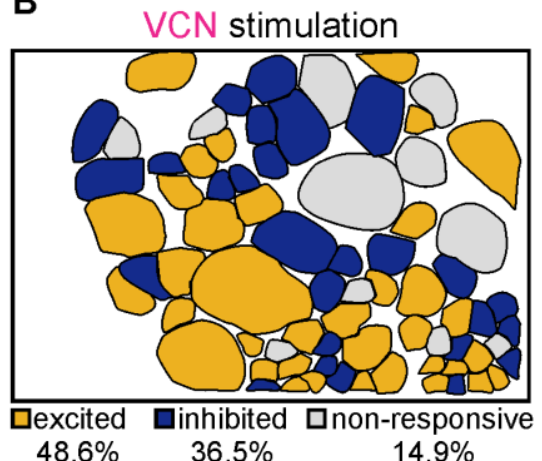

C

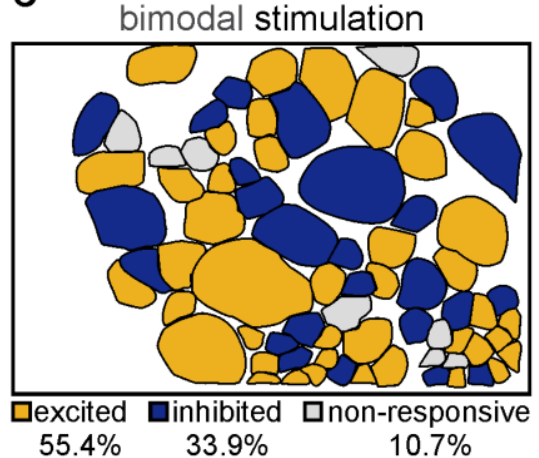

D

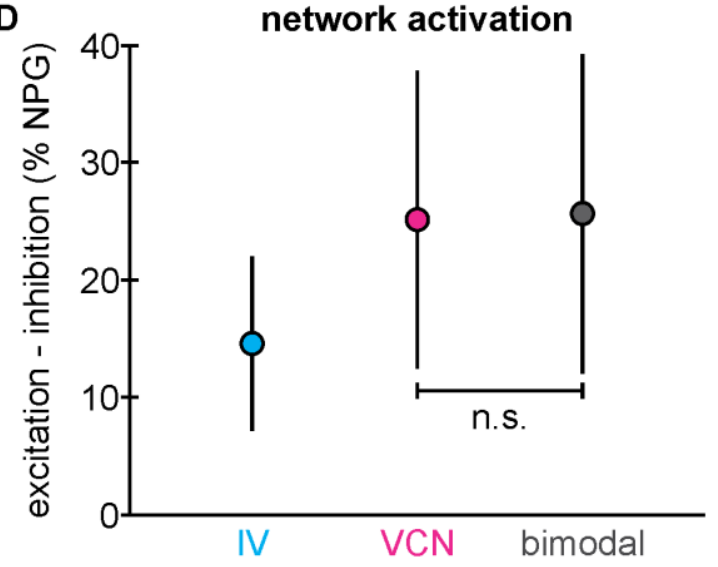

E

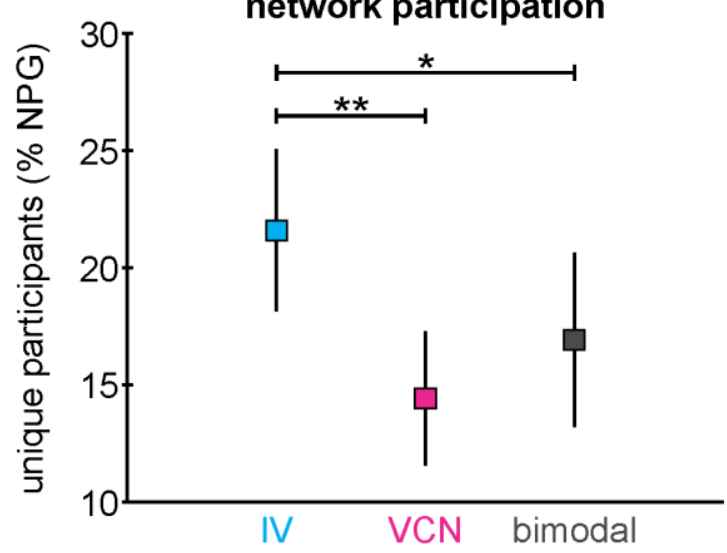

Fig. 7. Bimodal and mechanosensory stimuli yield similar premotor network activation and participation. (A) Example response map for CoG neurons during IV stimulation. (B) Example response map for $\mathrm{CoG}$ neurons during VCN stimulation. (C) Example response map for CoG neurons during bimodal stimulation. (D) Difference in the proportion of excited and inhibited neurons ('network activation') for IV, VCN and bimodal stimulation. No difference was found in premotor network activation between the $\mathrm{VCN}$ and bimodal conditions ( $\mathrm{N}=8$, n.s: not significant, repeated measures ANOVA with Tukey-Kramer post-hoc test). (E) Unique neuronal participants, defined as neurons responding uniquely in one condition and similarly in the other two conditions (i.e. excited by IV stimulation and inhibited by VCN and bimodal stimulations) for a given modality. There was a significantly larger proportion of unique participants associated with the IV condition when compared with the VCN and bimodal conditions $\left({ }^{*} p<0.05\right.$, repeated measures ANOVA with Tukey-Kramer post-hoc test). Any proportion of uniqueness greater than $0 \%$ indicates that the sensory condition was associated with some degree of unique neuronal participation. Each condition had a unique pool of neurons, but the $\mathrm{VCN}$ and bimodal conditions were similar in how unique their network responses were. For (A-C): excited neurons (yellow), inhibited neurons (blue), nonresponsive neurons (gray). Percentages below maps are reflective of the values measured for the example shown (not across preparations). 

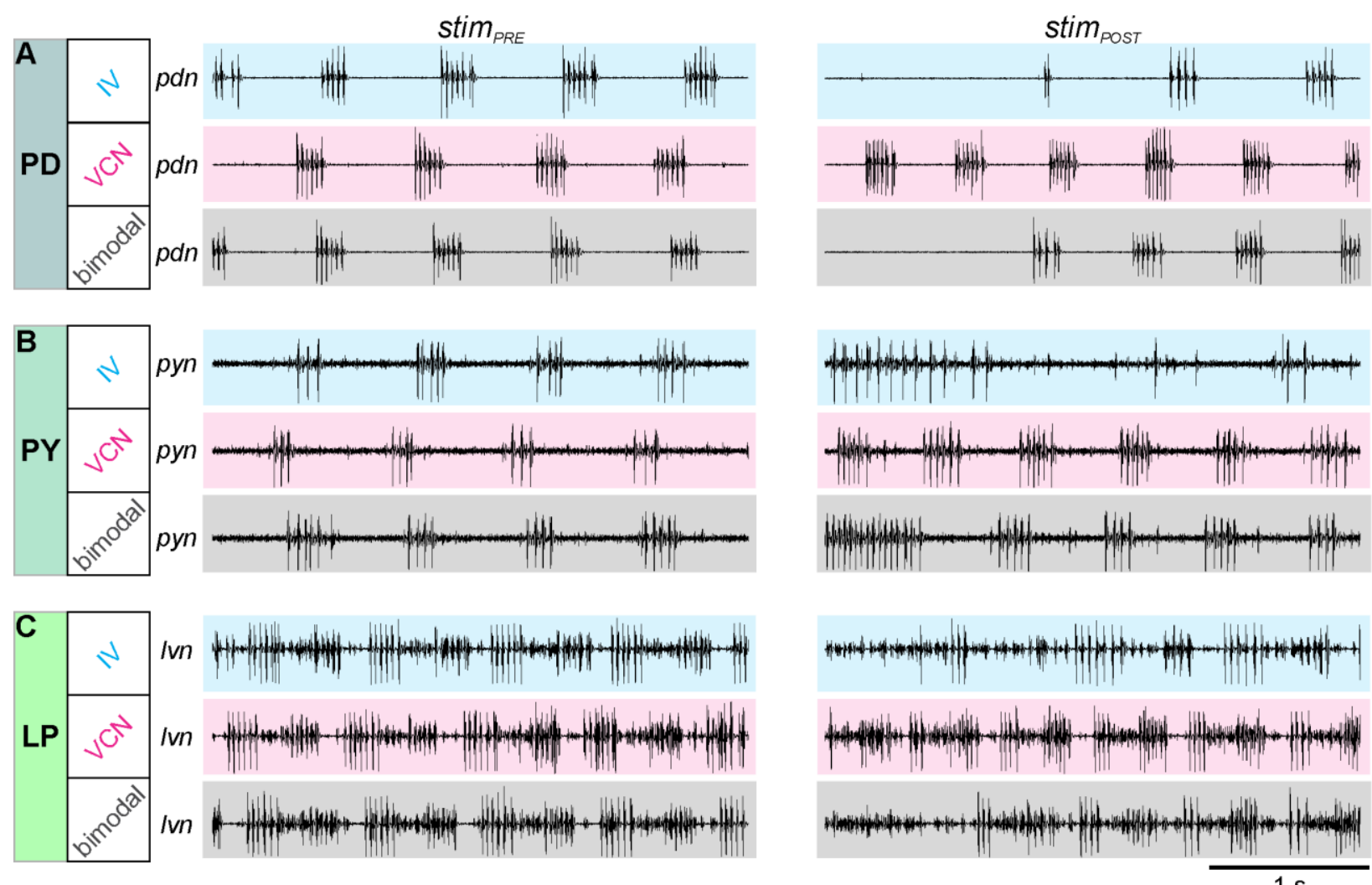

D firing frequency

E

F burst duration
G intraburst firing frequency

H
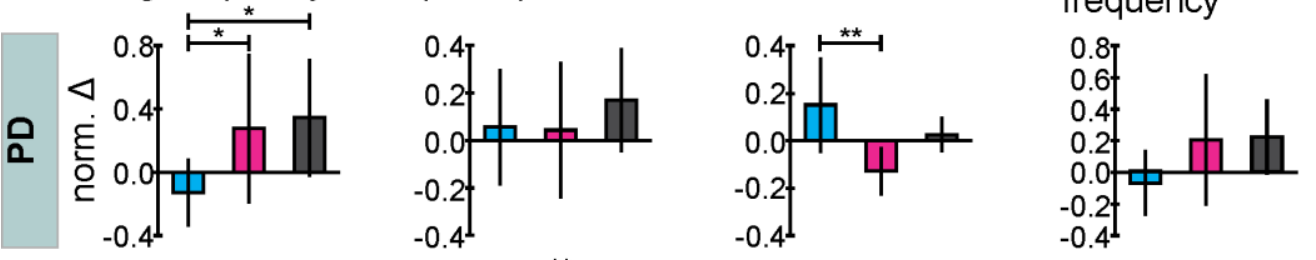

cycle period
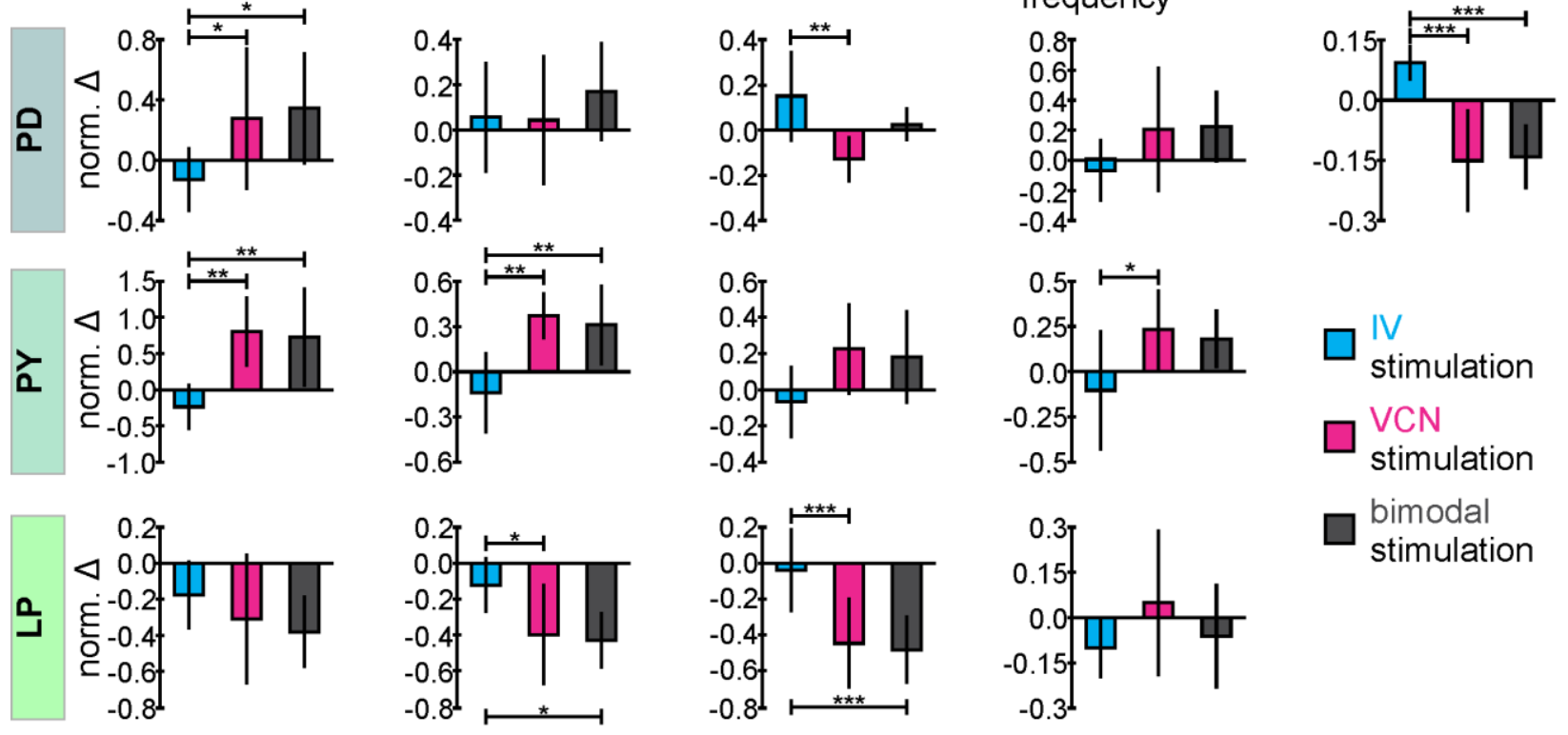

$\square$ stimulation
$\square$ VCN
stimulation stimulation stimulation

Fig. 8. Unimodal chemosensory and mechanosensory inputs yield functionally different pyloric motor patterns. (A-C) Sample traces of extracellularly recorded PD, PY and LP neuronal activity, respectively, immediately before (stimPRE, left) and after (stimPOST, right) sensory stimulation for IV unimodal input, VCN unimodal input, and bimodal input, as indicated. PD: pyloric dilator neuron, PY: pyloric constrictor neuron, LP: lateral pyloric 
neuron, pdn: pyloric dilator nerve, pyn: pyloric constrictor nerve, Ivn: lateral ventricular nerve. (D-H) Quantification of pyloric rhythm activity in response to IV unimodal (cyan bars), VCN unimodal (magenta bars), and bimodal (dark gray bars) input for PD (top plots), PY (middle plots), and LP (bottom plots). Neuronal firing frequency (D), number of spikes per burst $(E)$, burst duration $(F)$, and intraburst firing frequency $(G)$ were calculated separately for each neuron, while cycle period $(\mathrm{H})$ is a measurement reflective of the whole pattern. Data $(\mathrm{D}-\mathrm{H})$ are mean $\pm \mathrm{SD}$ of the normalized change in activity from stimPRE to stimPOST $\left(\mathrm{N}=8\right.$ for $\mathrm{PD}$ and $\mathrm{LP}, \mathrm{N}=7$ for $\mathrm{PY},{ }^{*} \mathrm{p}<0.05$, ${ }^{* *} \mathrm{p}<0.01$, ${ }^{* * *} p<0.001$, repeated measures ANOVA with Tukey-Kramer post-hoc test). 

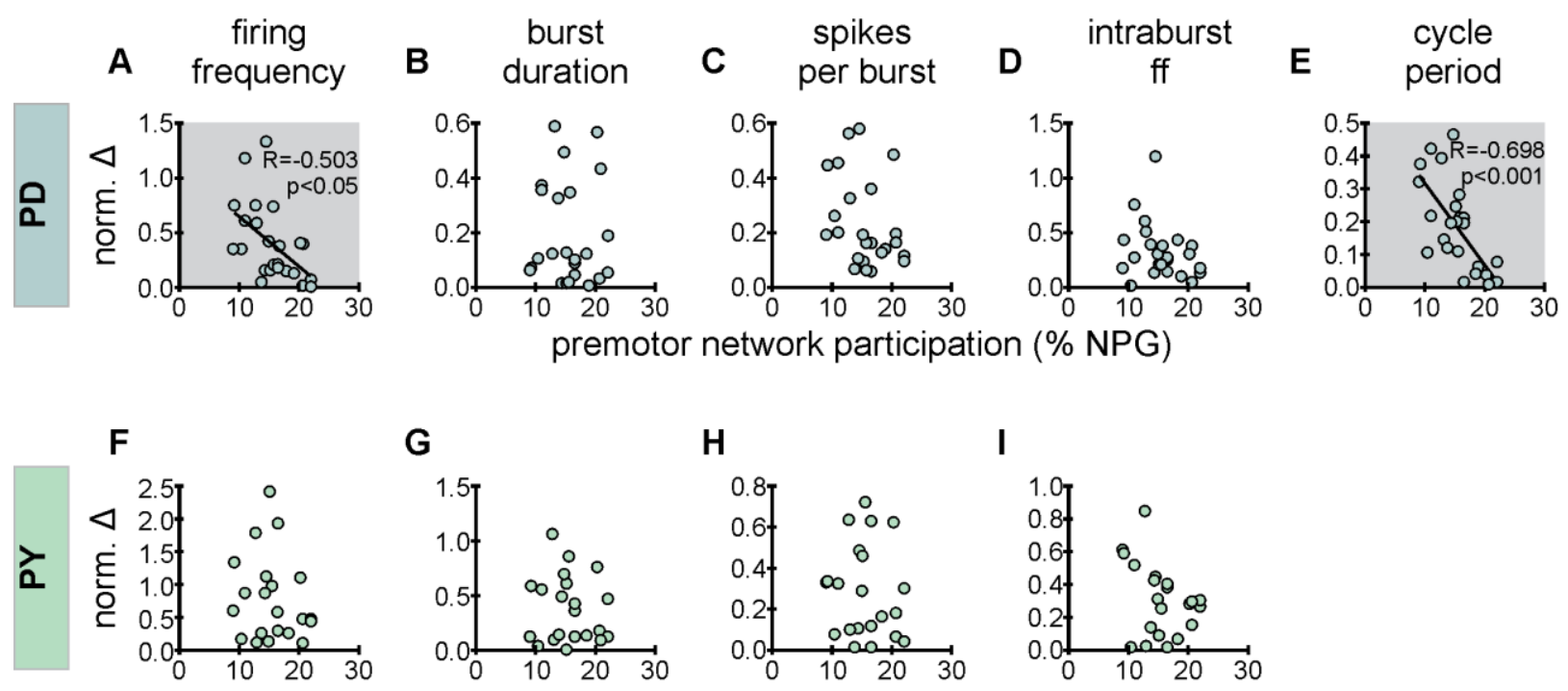

premotor network participation (\% NPG)
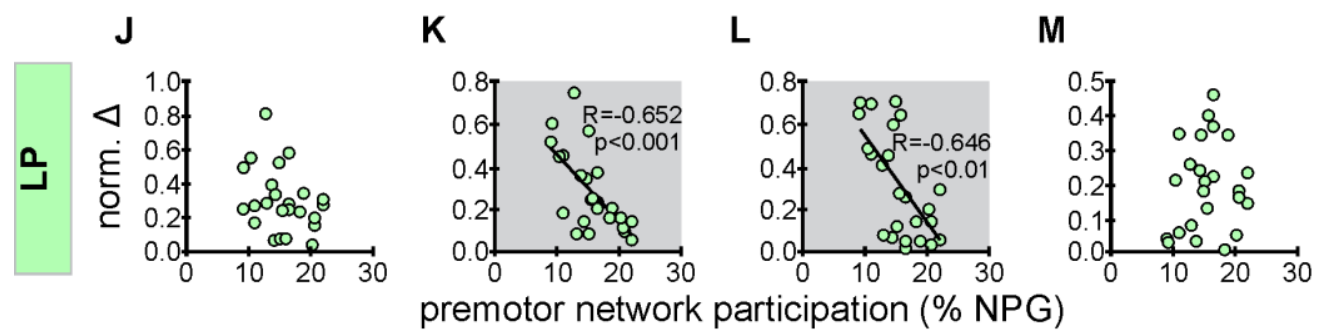

Fig. 9. Premotor network participation is related to the differences in pyloric motor output across sensory conditions. Correlation between similarities in premotor network participation and uniqueness in pyloric motor output activity are shown across animals (A-M). Uniqueness is the absolute difference between two sensory conditions' motor output: the larger this value is, the more unique the response of these two conditions are. Panels are organized as follows: PD (A-D), cycle period (E), PY (F-I), and LP (J-M). $(\mathrm{N}=24$ for $\mathrm{PD}$, cycle period, and LP; $\mathrm{N}=21$ for $\mathrm{PY}$; significant correlations are shown in shaded plots). 

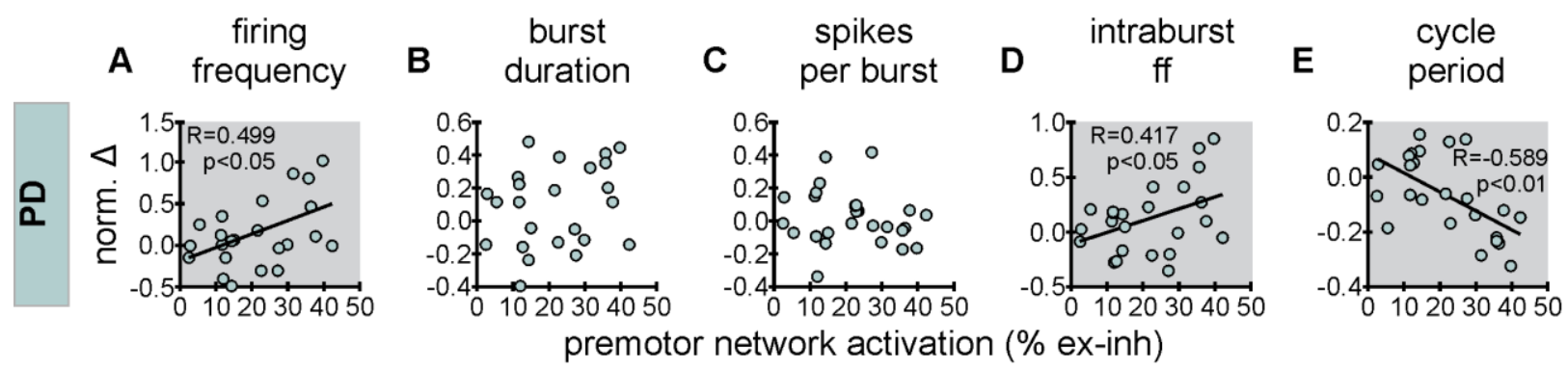

$$
\text { premotor network activation (\% ex-inh) }
$$
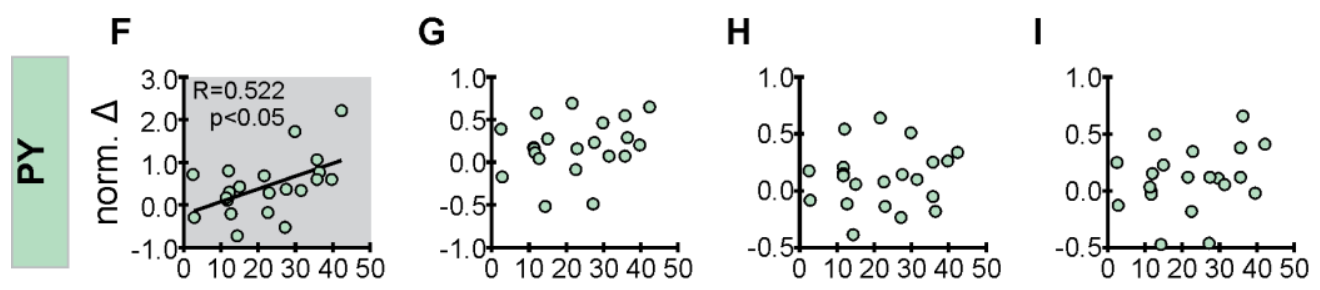
premotor network activation (\% ex-inh)

$\mathbf{J}$

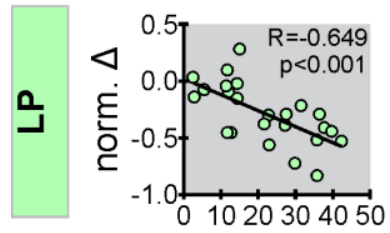

K

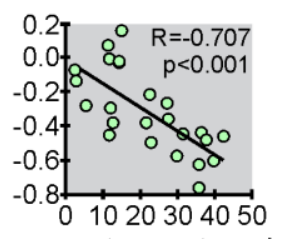

$\mathbf{L}$

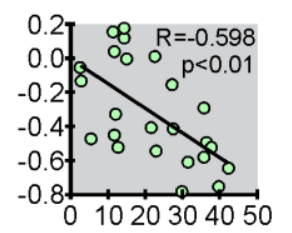

M

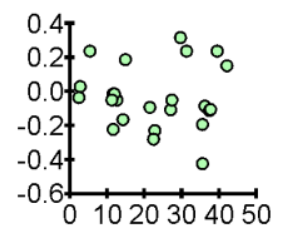
premotor network activation (\% ex-inh)

Fig. 10. CoG premotor network activation is related to the change in downstream pyloric activity. Scatter plots of premotor network activation (the difference in the proportion of excited neurons and inhibited neurons) versus changes of the pyloric motor output (firing frequency, burst duration, spikes per burst, intraburst firing frequency, and cycle period) across animals reveal relationships between premotor and motor activity: PD (A$D)$, cycle period (E), PY (F-I), and LP (J-M). All comparisons were made using Pearson correlation ( $N=24$ for $P D$, cycle period, and $L P ; N=21$ for $P Y$; significant correlations are shown in shaded plots). 
A
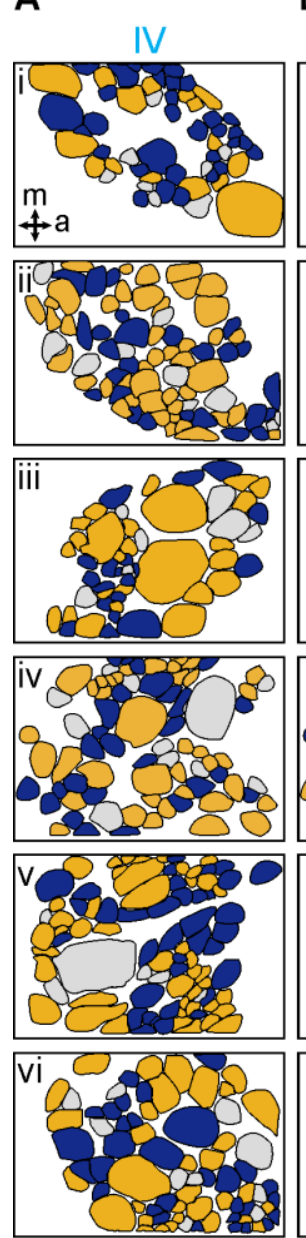

$\square$ non-responsive
B
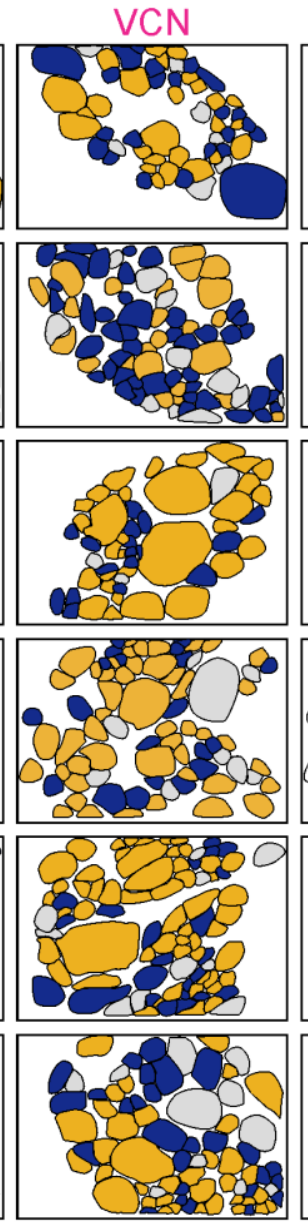

$\square$ excited
C
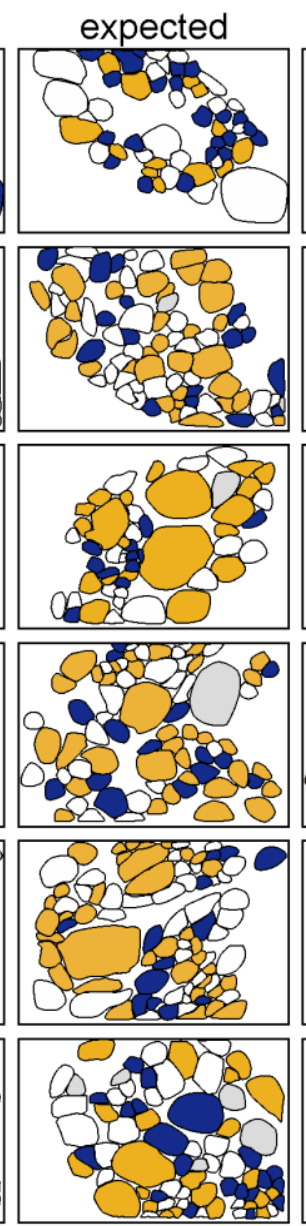

a inhibited
D
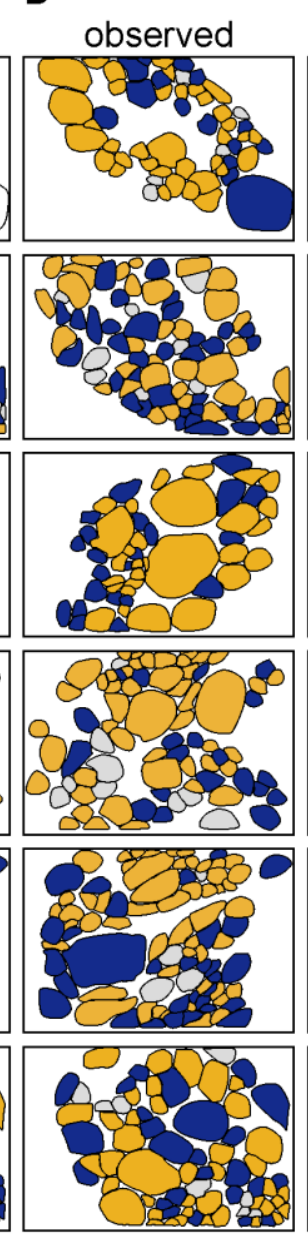

$\square$ no rule
E congruence
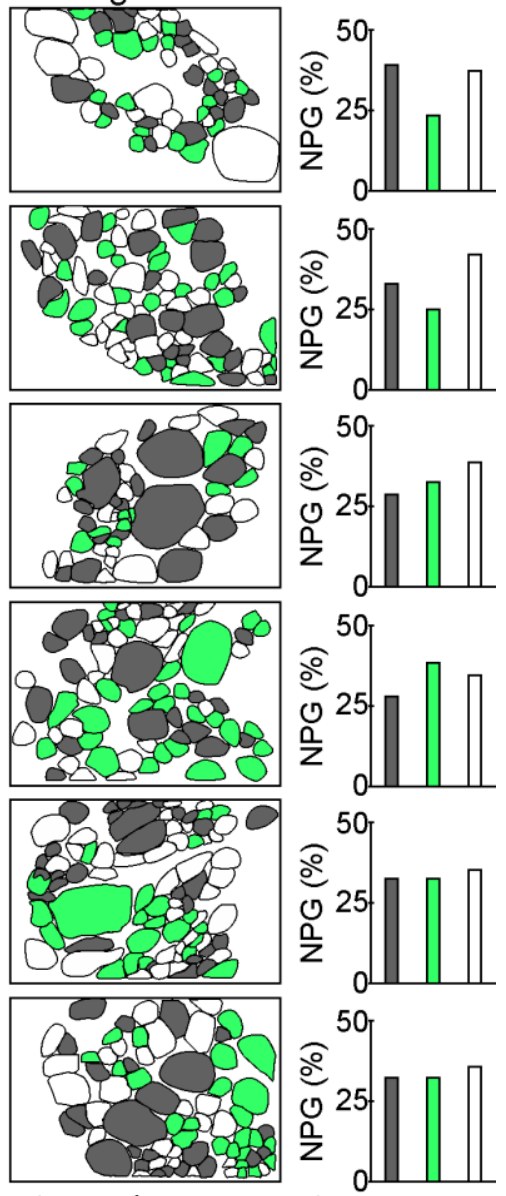

Fig. 11. CoG Network response to bimodal sensory stimulation differs from expected outcomes. (A and B) Maps of excited (yellow cell bodies), inhibited (blue cell bodies), and non-responsive (light gray cell bodies) neurons across six ganglia (i-vi) during IV (A) and VCN (B) stimulation. (C) Map of expected outcomes for bimodal IV and VCN stimulation, based on additive responses ruled from the unisensory responses, and additional scenarios where no expectations could be formulated ("no rule", white cell bodies). The set of expected outcomes for additive responses during bimodal stimulation of IV and VCN pathways were extrapolated based on additive responses as follows (see also Materials and Methods): an excitatory response to both unimodal stimuli is expected to result in excitation during bimodal stimulation ( $/ \mathrm{V}_{\mathrm{ex}} / \mathrm{VCN} \mathrm{ex}=$ bimodal outcome $\left.\left(\mathrm{CO}_{\mathrm{ex}},\right)\right)$. Similarly, an inhibitory response to each modality should yield an inhibited bimodal response ( $\left(\mathrm{V}_{\mathrm{inh}} / \mathrm{VCN}\right.$ inh=COinh). No response to either modality should result in no bimodal response (IVNR/VCNNR=CONR), and an excitatory response to one unimodal stimulus and no response to the other would lead to an excitatory bimodal response $\left(\mathrm{IV}\right.$ ex $/ V C N_{N R}=\mathrm{CO}_{\mathrm{ex}}$; IV $\left.\mathrm{NR}_{\mathrm{NR}} / \mathrm{VCN}_{\mathrm{ex}}=\mathrm{CO}_{\mathrm{ex}}\right)$. An inhibitory response to one unimodal input and no response to the other would result in an inhibitory bimodal response $\left(\mathrm{IV}_{\text {inh }} / \mathrm{VCN} \mathrm{NR}_{\mathrm{NR}}=\mathrm{CO}_{\text {inh; }}\right.$; IV $\left.\mathrm{NR}_{\mathrm{NR}} / \mathrm{VCN}_{\text {inh }}=\mathrm{CO}_{\text {inh }}\right)$. Two scenarios $\left(\mathrm{IV}_{\mathrm{ex}} / \mathrm{VCN}_{\text {inh }}\right.$ and 
I $\mathrm{V}_{\text {inh }} / \mathrm{VCN}_{\text {ex }}$ ) cannot directly predict the additive outcome, because the potential outcome depends on the strengths of the excitation and inhibition. These scenarios are dubbed "no rule" (white). (D) Experimentally acquired neuronal responses during bimodal IV and VCN stimulation (observed outcomes). (E) Map of congruency between expected (C) and observed (D) outcomes, illustrating the agreement between the expected and observed cases. Dark gray: neurons whose observed and expected outcomes matched ("congruent"). Green: neurons whose expected and observed outcomes did not match ("incongruent"). White: neurons with no clear expected outcomes ("no rule", IV ex $/ V^{\prime} \mathrm{N}_{\text {inh }}$ and $I V_{\text {inh }} / V_{C N}$ ex), which was the case for $37.19 \pm 2.87 \%$ of all neurons imaged (white cell bodies, $n=159 ; N=6)$. (F) Proportion of neurons for each ganglion with congruent (dark gray) and incongruent (green) bimodal responses, and where no rule applied and thus no expectations could be made (white). 